\title{
Optimization Based Decision Support Tools for Fire and Rescue Resource Planning
}

\section{Anna Ulander}

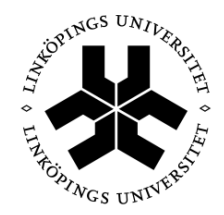

\section{Linköping University} INSTITUTE OF TECHNOLOGY

\author{
Department of Science and Technology \\ Linköping University \\ SE-601 74 Norrköping, Sweden
}

Norrköping 2015 


\section{Optimization Based Decision Support Tools for Fire and Rescue Resource Planning}

(C) Anna Ulander, 2015

Cover illustration by Maria Jitzmark

ISBN 978-91-7519-132-4

ISSN 0280-7971

Linköping University

Department of Science and Technology

SE-601 74 Norrköping

Printed by LiU-Tryck, Linköping, Sweden 2015 


\section{Abstract}

When accidents occur, it is essential that fire and rescue service respond quickly and efficiently to the accident site to reduce suffering and save lives and property. Planning and coordinating of fire and rescue resources is therefore important in order to maintain a safe society. Firefighters in Sweden have by tradition worked in teams of five, and they have been allocated to fire stations strategically located in populated areas. However, this working approach has recently started to change and the fire and rescue services have started to deploy smaller groups of firefighters. These smaller units can be, for example, strategically located near high-risk areas or roads, or used for preventive work. The complexity of the resource planning process thus increases since the decision makers have to keep track of a large number of small units spread over the area. The new way of working has resulted in an increased need of support tools that can help the fire and rescue services in decisions regarding the resource management.

In this thesis, optimization based decision support tools are developed in order to to support the fire and rescue service so they can efficiently manage, coordinate and dispatch fire and rescue resources with respect to the present demand for service.

To find appropriate strategic and tactical locations for different types of response units, an optimization model that minimizes the response time to expected accidents is developed. The model considers both the response time for the first responding unit as well as the response time for the last responding unit. Furthermore, the model is flexible enough to incorporate any type of accidents or resources. The results show that the model can be used to produce practical support for various types of location decisions.

To support the more complex operational planning, three optimization based decision support tools are developed. The tools can help the fire and rescue service to always maintain an adequate level of preparedness for handling accidents. To evaluate the preparedness, a quantitative measure has been defined and serves as the basis for the tools. The first tool can help illustrate the preparedness, the second tool to select appropriate vehicles and firefighters to dispatch to accidents, and the third tool to suggest how resources can be relocated to maintain an adequate preparedness for new accidents.

The developed decision support tools are evaluated through tests and experiments with fire and rescue services in Sweden. Results from the experiments indicate that the three tools can support the fire and rescue service in operational decisions and in maintaining an adequate preparedness for handling accidents, but also that the planning time seems to increase when using the support tools. However, the participants' experiences of the tools were in general positive, and they thought the tools were useful and that the tools could support their daily work. The perception of preparedness, which usually varies quite a bit among individuals, also seems to become more uniform with access to the tools. 


\section{Acknowledgement}

First and foremost I am very grateful I got the opportunity to perform this work at the division of Communications and Transport Systems (KTS) at Linköping University, Campus Norrköping. Many people have given me help and support to finalize this thesis.

I would like to thank my main supervisors, Professor Jan Lundgren and Associate Professor Tobias Andersson Granberg for all guidance and support during the whole research process, and also for reading and providing feedback on my work. Without you, this would not have been realized.

I appreciate the financial support from Myndigheten för Samhällsskydd och Beredskap (MSB), and Räddningstjänsten Östra Götaland.

Special thanks to Räddningstjänsten Östra Götaland who have taken their time to support me with valuable information and statistical data. Thanks for the workshops and discussions along the way.

I want to thank Rego Granlund and Jonas Lundberg for an enjoyable collaboration in the research project DYRK (Dynamisk Planering av Responssystemet i Kommunen). The project was a collaboration between Linköping University, SICS East Swedish ICT AB and MSB. Thanks Rego for all interesting discussions during our experiment tours and also for being a good friend.

I also want to show my appreciation to all the fire and rescue services and organizations we have interviewed and who have participated in our series of experiments. Special thanks to the fire and rescue services in Göteborg, Hudiksvall, Järfälla, Jönköping, Kalmar, Kisa, Kristianstad, Lindvreten, Linköping, Lund, Malmö, Norrköping and Nässjö.

Thanks to all my colleagues at KTS who have provided a stimulating and enjoyable environment to work in. Special thanks to Åsa Weinholt and Ngoc-Hien Thi Nguyen for reviewing some parts of this work. All your constructive comments have been helpful and valuable. I would also thank my former colleague Maria Johansson who has made my days and lunches extra joyful.

Finally, I would express my gratitude to my family and my friends for always supporting me and believing in me in every way possible throughout my life. Special thanks to my brother Andreas Gustafsson for all good advice and recommendations regarding C\# programming. Thanks also to my auntie Maria "Mimmi" Jitzmark for the beautiful drawing for the cover page.

Last but not least, I am most grateful to my lovely husband Daniel for providing love and inspiration of the best kind, and our little unborn child who has started to kick me just as a reminder I must prepare myself for becoming a mother

Norrköping, February 2015 Anna Ulander 


\section{List of Contents}

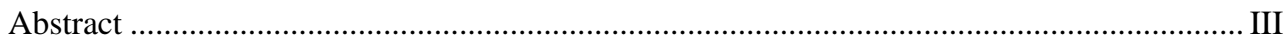

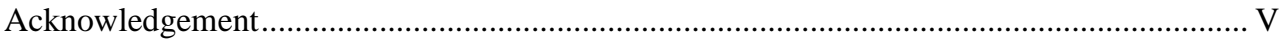

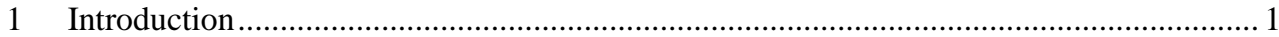

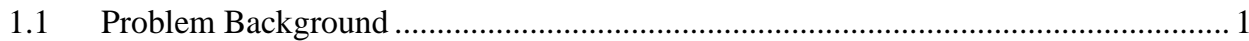

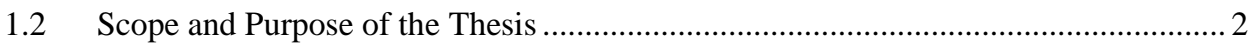

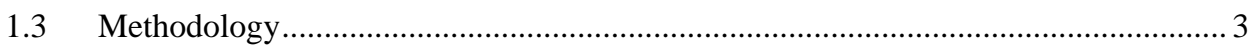

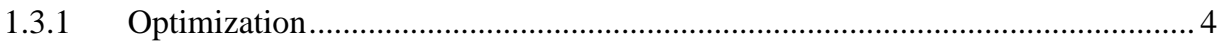

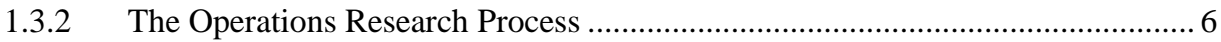

1.3.3 Description of the Thesis's Working Process .................................................... 7

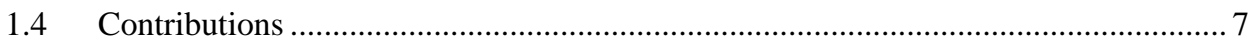

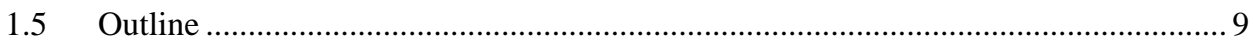

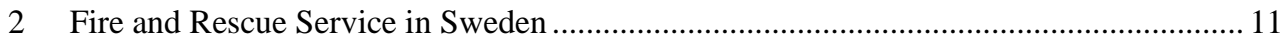

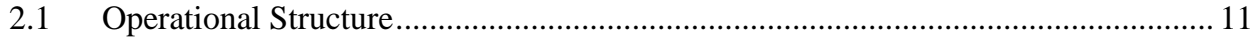

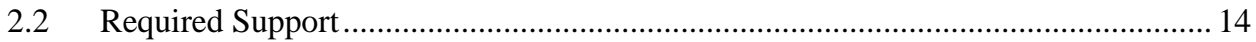

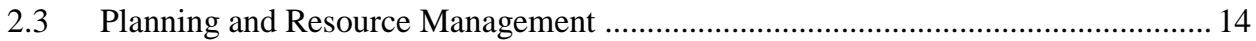

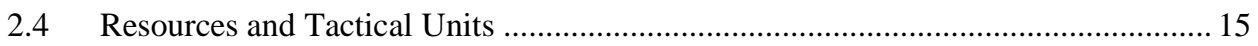

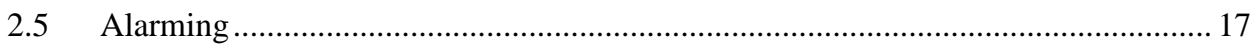

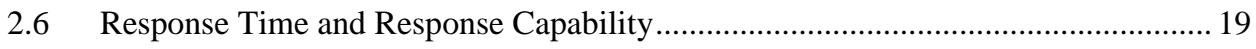

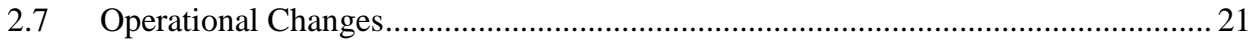

2.8 How the Operational Changes Influence the Fire and Rescue Service ....................26

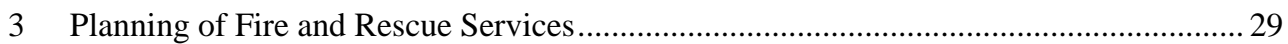

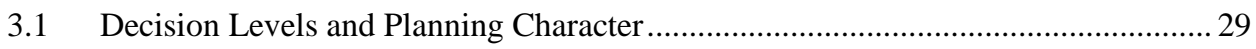

3.2 Planning Challenges within Fire and Rescue Services........................................ 31

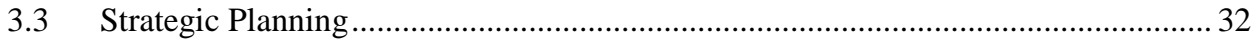

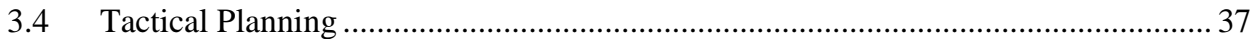

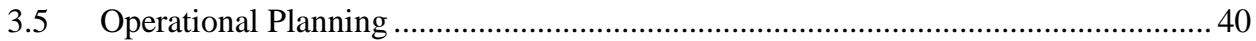

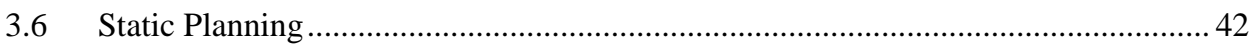

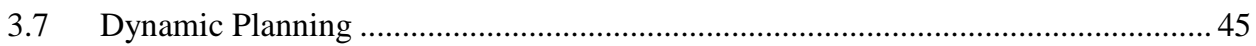

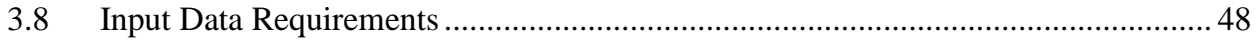

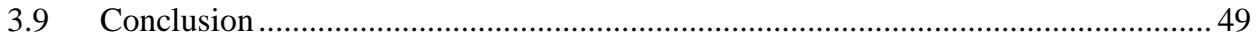

4 Strategic Location of Fire and Rescue Resources......................................................... 51

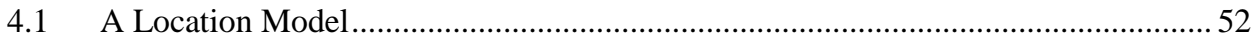




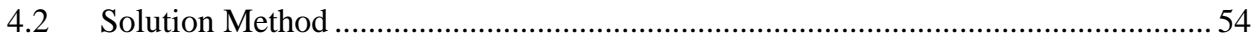

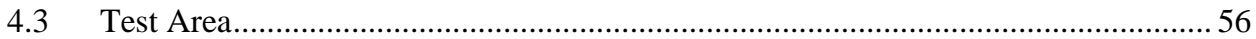

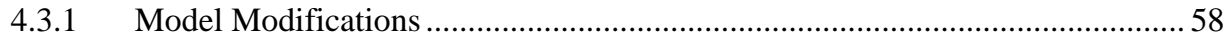

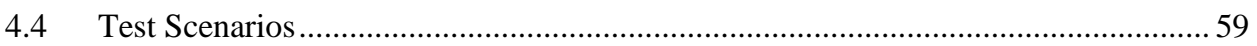

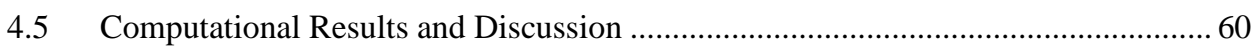

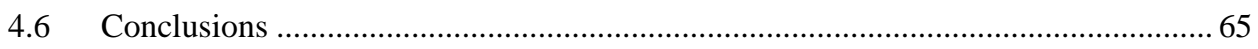

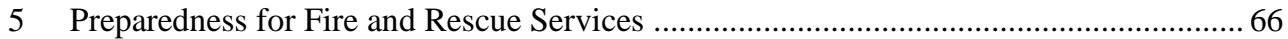

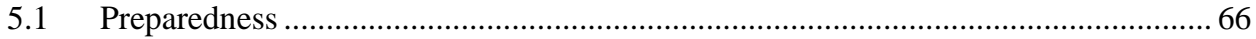

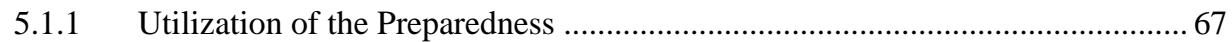

5.1.2 Preparedness Estimation and Indicators that affect the Preparedness................ 69

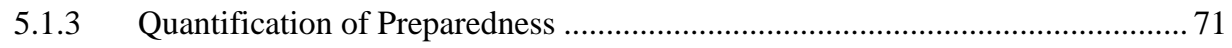

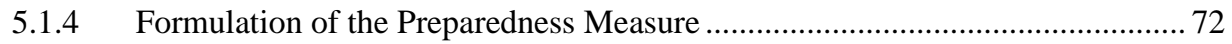

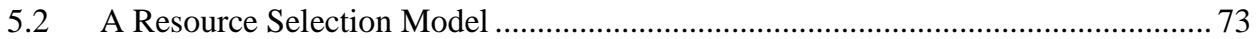

5.3 Solution Method for the Resource Selection Model ................................................. 76

5.3.1 The Heuristic Developed for Solving the Resource Selection Model................. 76

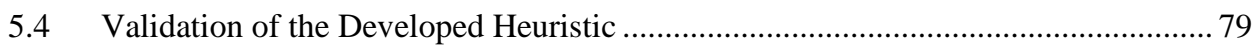

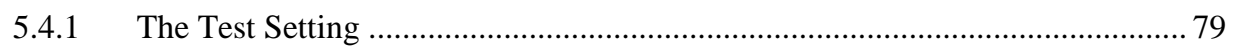

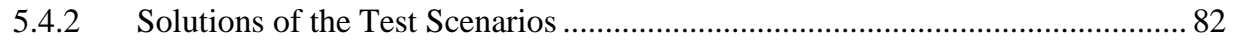

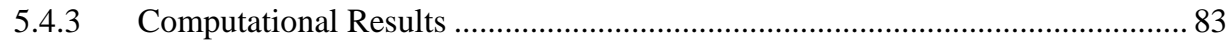

5.5 Calibration and Validation of the Preparedness Value ............................................. 85

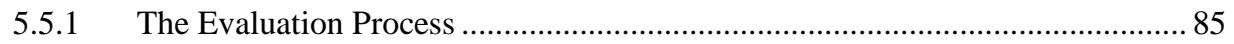

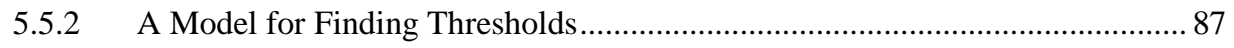

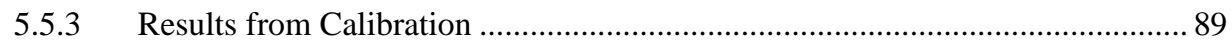

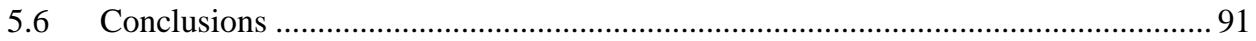

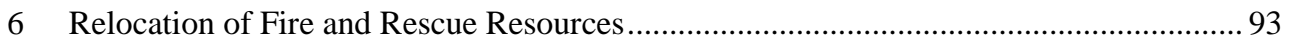

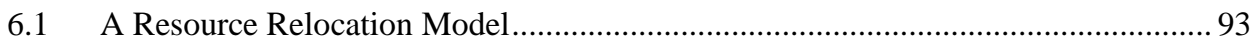

6.2 Solution Method for the Relocation Model .............................................................. 99

6.2.1 The Heuristic Developed for Solving the Relocation Model ............................ 99

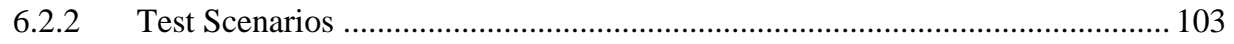

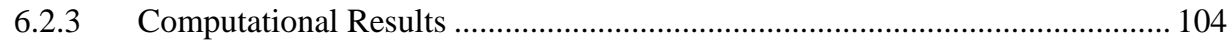

6.2.4 Validation of the Developed Heuristic ................................................................... 107

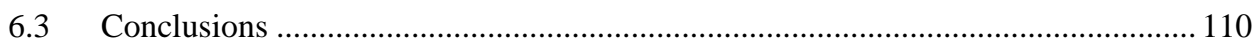

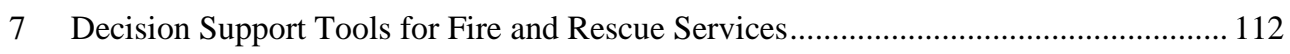




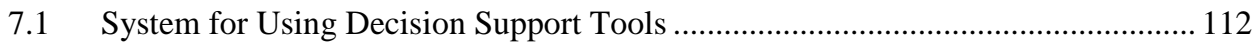

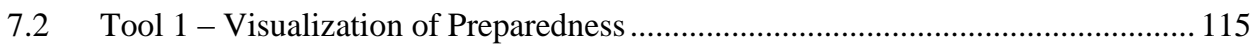

7.3 Tool 2 - Dispatching of Resources to Accidents ................................................... 116

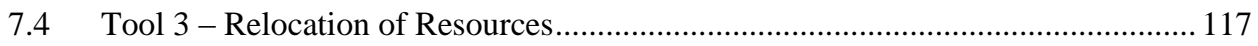

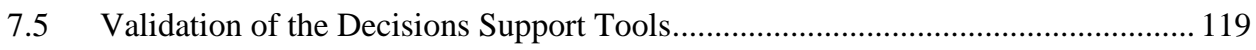

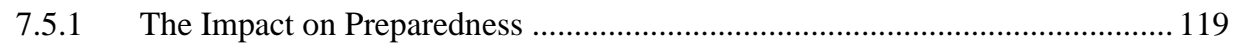

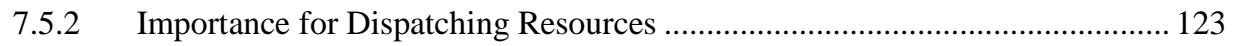

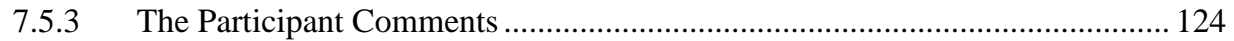

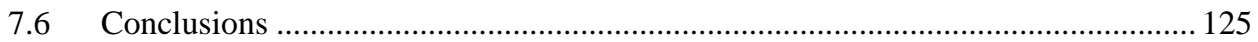

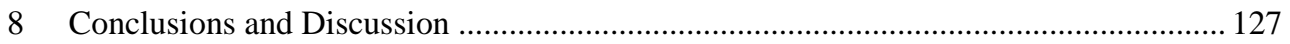

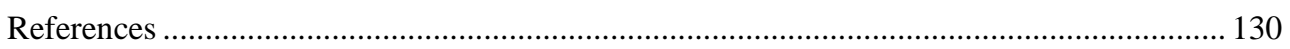

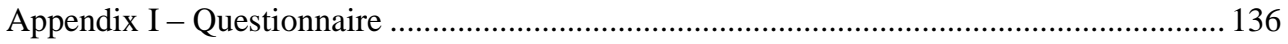




\section{Introduction}

When accidents occur, it is essential that the fire and rescue service respond efficiently to the accident site. It is usually important with quick responses to reduce suffering and save lives and property. Essential is also to make sure that the right type of resources and skilled personnel arrive at an accident to be able to perform the required emergency operations. Thus, planning and coordinating of fire and rescue resources is important in order to maintain a safe society.

Since accidents can occur at any time and the demand for service varies with time and space, the fire and rescue service must coordinate and allocate resources in order to all the time be able to respond to new incoming accidents, i.e. to maintain an adequate preparedness. In parallel to performing adequate urgent responses to incoming accidents, the fire and rescue services must also be able to work proactively to reduce risk situations and prevent accidents. Decisions must often be made quickly, and it is thus essential with adequate planning to manage and organize the operations. The challenge is often to ensure that there are always enough resources with the right expertise, that the resources are located where they currently are needed and that various non-urgent tasks are performed when appropriate. Another main challenge is to decide which resources to dispatch to a specific accident.

\subsection{Problem Background}

The fire and rescue service in Sweden has traditionally been characterized by relative static planning and resource management. The firefighters have, for example, been allocated to fire stations that have been strategically located in populated areas. The firefighters have also usually worked in teams of four firefighters and one fire officer with the fire station as a base. This means the resources have not always been optimally located in relation to demand. If, for example, a fire station is depleted of resources, the preparedness in the surrounding area may become inadequate. This issue has been observed by Myndigheten för Samhällsskydd och Beredskap (MSB), (the Swedish Civil Contingencies Agency).

The fire and rescue services should be able to coordinate and allocate resources in order to always maintain an adequate preparedness. The fire and rescue services' working approach in Sweden has, thus, slowly started to change in order to improve the service quality and efficiency. The trend seems to be moving to work with smaller groups of firefighters that also are more geographically dispersed, i.e. they are not necessary located at fire stations. Some firefighters can, for example, perform different preventive tasks while others are strategically located at various places, e.g. high-risk areas or roads, in order to maintain a good level of preparedness. The planning process has, thus, started to become more dynamic in order to adapt the fire and rescue service to variations in demand.

The new working approach is meant to provide a better flexibility to respond to incidents. When resources are more widespread, the probability that they are available where they are needed will increase. However, the process of coordinating and allocating resources becomes more complex. When a decision maker must keep track of a large number of smaller groups of firefighters spread over the area as well as their competences, it becomes a tougher 
challenge to evaluate the ability to respond to new incidents. Two or several smaller groups of firefighters can, for example, be dispatched from different places and form a rescue team at the accident site. It, thus, becomes more complicated to make decisions regarding which resources should be dispatched to an accident and how resources should be located to maintain an adequate level of preparedness.

The new situation has led to an increased need for support tools that can help the fire and rescue service to coordinate and allocate resources and to make important decisions regarding where resources should be located to best meet the demand for service and how resources should be dispatched to incidents. Support tools to provide a good overview of all the resources is also of importance.

\subsection{Scope and Purpose of the Thesis}

The overall purpose of this thesis is to develop optimization based decision support tools to support the fire and rescue service so they can efficiently manage, coordinate and dispatch fire and rescue resources with respect to the present demand for service.

Since it is important that fire and rescue resources can quickly reach accidents, it is essential that resources are located where they can coordinate efficient responses. In this thesis, an optimization model that locates fire and rescue resources in relation to expected demand is developed. The characteristic feature of the developed location model is that it in particular considers the first responding resources' response time, which may also be valued differently in relation to the remaining resources' response time. The aim with the developed location model is to evaluate the strategic location of a given number of fire and rescue resources in a given area in order to minimize the response time to different types of accidents. The model can be used to locate multiple types of response units, i.e. different combinations of vehicles and firefighters with respect to different types of accidents. The model has been used for solving the location problem for a number of scenarios with different characteristics.

The thesis also presents and describes the development of decision support tools intended for operational planning. Three tools are developed in order to facilitate and support the more complex planning process of managing fire and rescue resources. Since one of the main objectives for the fire and rescue services is to continuously maintain an adequate preparedness for accidents, a quantitative measure for calculating the preparedness is defined and serves as a basis for the developed decision support tools. The measure is a function of the response time for the resources that are requested to a given incident and the probability that the incident will occur.

The three tools that are developed are as follows: one that illustrates the preparedness, i.e. a preparedness visualizer, for a given type of accident, one that gives suggestions on which resources that are most appropriate to dispatch to an accident in order to minimize the response time and one that gives suggestions on how resources can be relocated in order to improve the preparedness, i.e. in order to best be available if an accident occur. The tools consider multiple types of accidents as well as multiple types of resources. 
The decision support tools are based on two optimization models that are developed in the thesis. It is one resources selection model and one resource relocation model. The resource selection model is developed in order to determine the most appropriate resources that are needed for a given type of accident. The resources must be determined to be able to calculate the preparedness with the defined measure. The relocation model is developed to give suggestions of how resources in an area can be relocated to new positions in order to improve the preparedness in the area. The model gives suggestions on which resources to relocate and where to relocate them.

To practically be able to use the tools, the underlying optimization models are connected to a map-based system, C3Fire, which is a geographical information system (GIS), and a user interface, SMOKE, which is a computer-based system for handling the resources.

The decision support tools are evaluated through two series of experiments performed with a number of fire and rescue services in Sweden. In the experiments, fire and rescue service personnel made decisions regarding how resources should be coordinated and used in various situations and with respect to the demand for service.

\subsection{Methodology}

The methodology used in this thesis belongs to the operations research (OR) area. OR is an empirical science that deals with applications of advanced analytical methods to help make better decisions and it refers to quantitative analysis, where quantification and measurability are required for any result to be obtained. Usually mathematical models are created, solved and tested to analyze complex situations to make intelligent decisions and conclusions (Larsson, 2004).

An example of a definition of operations research is "preparation of basis for rational decisions using systematic scientific methods and - where possible and meaningful - of quantitative models" (SOAF, 2012).

To plan and improve emergency response capabilities, operations research has proven to be an efficient and well-used method to support the planning process, especially when it comes to decision making and planning to achieve effective and efficient use of the emergency resources. Operations research has been applied to many kinds of problems in the emergency service area, and often to facilitate decision making and different types of planning in order to improve the rescue effort. A variety of problems, such as locating emergency service facilities, scheduling personnel, allocating emergency vehicles or designing emergency response systems have been solved with a number of different approaches and models (Goldberg, 2004; Simpson and Hancock, 2009; Swersey, 1994).

Quantitative research analysis is made using mathematically based methods. Examples of quantitative methods are optimization and simulation. The quantitative method used in this thesis is optimization, which is described in the following section. 


\subsubsection{Optimization}

Scientifically, optimization theory is about to develop and solve a mathematical model and find a good, or preferably the proven best, solution to the problem given certain conditions and restrictions. An optimization problem is usually expressed in the form of an objective function and a number of constraints. In general, it is about finding "the best available" value of the objective function and then the evaluation of a solution will be numerically performed.

The objective function and the constraints consist of variables and parameters. The constraints establish the conditions and restrictions and usually consist of equality and inequalities between functions of variables and parameters. The optimization problem is to find a feasible solution of variables and parameters, compatible with the constraints that minimize (or maximize, depending on the problem and the goal) the objective function for the given values of parameters. The objective function is also normally called cost function if the goal is to minimize the objective function or utility function if the goal is to maximize the objective function. The feasible solution that minimizes (or maximizes) the objective function is then called an optimal solution (Bertsimas and Tsitsiklis, 1997).

Practically, optimization may refer to improvement, speeding up or streamlining in different contexts. It is about, from some sort of starting position, performing something better.

In optimization theory, different models are used to set up and solve concrete problems. Usually you distinguish between different types of models depending on the type of optimization problem you will solve (Lundgren et al. 2010). Linear programming (LP) problems examine cases in which the objective function is linear, all the relations between the variables are linear and the set of constraints is specified using only equalities and inequalities. Integer programming (IP) problems study linear problems in which all variables are constrained to take integer values. In the special case of integer programming where all the variables are binary, the problem is called a binary integer programming (BIP) problem. Nonlinear programming (NP) problems study the general case in which the objective function or the constraints or both contain nonlinear relations. For solving and making quantitative analyses of the studied fire and rescue service problems included in this thesis, binary integer programming (BIP) is used.

IP problems are not convex, and are in general much more difficult to solve than regular LP problems. There are some general solution strategies that can be used for finding exact solutions to IP problems, for example relaxation methods, cutting plane methods and branch and bound methods (Lundgren et al. 2010). However, a general method cannot always be used for finding an exact solution to an IP problem. For very hard optimization problems when the model is too complex for using standard methods to find an exact solution or if the method is too slow, it can be helpful to use heuristic methods.

Heuristics is a class of methods that includes many types of solution approaches, from methods based on very simple rules of thumb to methods based on advanced optimization techniques. Heuristic methods often generate good solutions within a reasonable amount of time, but without any guarantee of identifying the optimal solution. The solution is often near- 
optimal, but it is not possible to measure how close. However, a heuristic solution is usually good enough because its synthesis is not prohibitively protracted. Heuristics is often used to solve difficult optimization problems, for example IP problems (Burke and Kendall, 2005; Lundgren et al. 2010; Michalewicz and Fogel, 2004).

There are many heuristic methods to choose between when solving a problem. Commonly used heuristic methods are, for example, greedy based heuristics and local search. A greedy based heuristic is a heuristic that simply selects the best available option in every step (Michalewicz and Fogel, 2004). In local search, the procedure starts from an initial solution and then uses different neighborhoods for finding new candidate solutions. Local search algorithms move from solution to solution in the search space by applying local changes. This continues until a solution has been found that has no better solution in its neighborhood, i.e. a local optimum is found (Michalewicz and Fogel, 2004). For more advanced search methods, metaheuristics can be used. Metaheuristics can be applied to a broad range of combinatorial problems. Examples of traditional metaheuristics are tabu search, simulated annealing and variable neighborhood search (Burke and Kendall, 2005). While local search methods have a tendency to become stuck in suboptimal regions, the exemplified metaheuristic methods are based on the idea of escaping from local optima in the hope of finding an improved solution. Ant colony optimization and genetic algorithm are other examples of metaheuristics where the search strategy has a learning component within the search procedure (Burke and Kendall, 2005). Another example of a metaheuristic is the greedy randomized adaptive search procedure (GRASP) heuristic, which consists of iterations made up from successive constructions of greedy randomized solutions and subsequent iterative improvements of the solution through local search (Resende and Silva, 2009). To determine whether a particular heuristic helps or hinder, the method has to be thoroughly tested.

For solving the models that are developed in this thesis, reduced variable neighborhood search and greedy based heuristics are used. 


\subsubsection{The Operations Research Process}

The method for how a research problem can be approached and solved when using operations research (OR) and optimization as the quantitative method for solving the problem, can be described as the process presented in Figure 1 (Kothari, 2004).

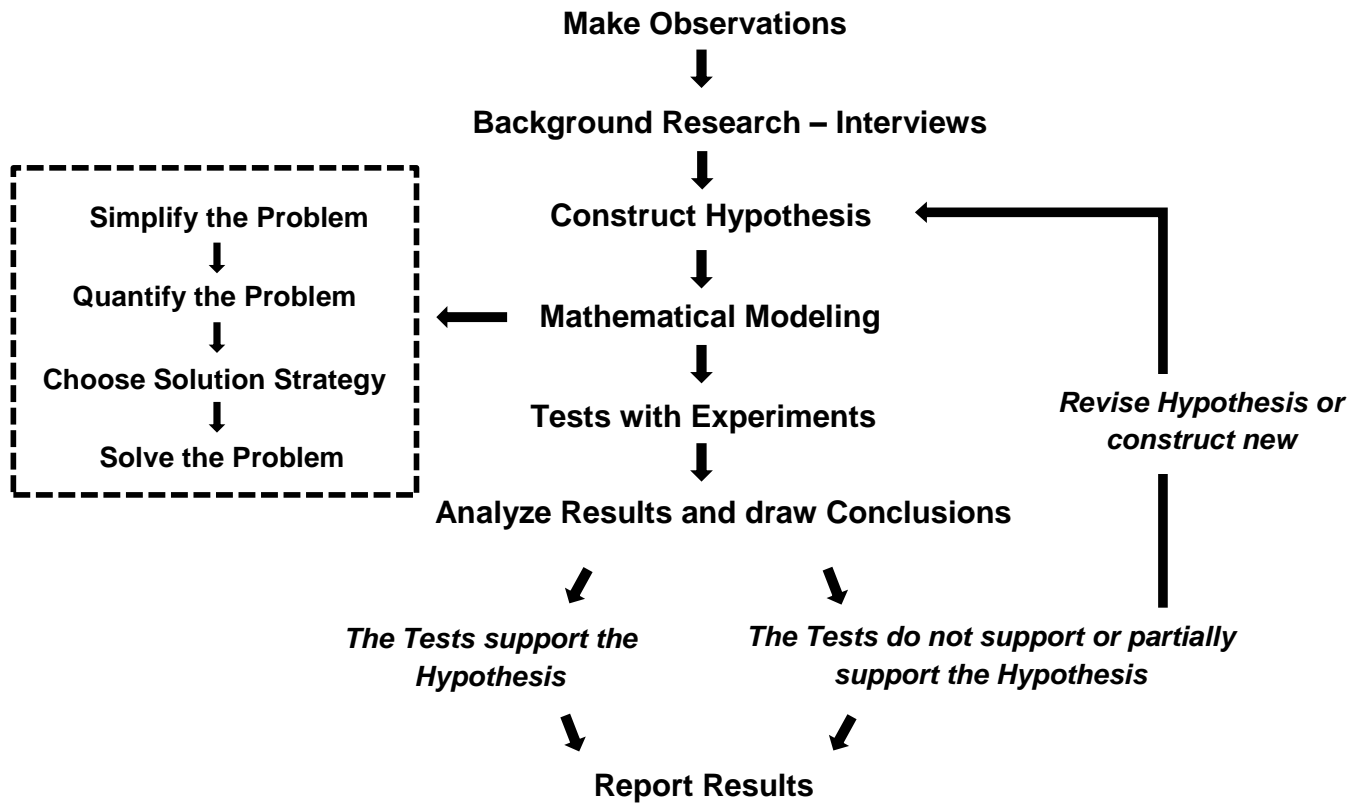

Figure 1. The operations research process used for the research in this thesis. (Kothari, 2004)

First, general observations about the problem are usually made. Then, more detailed investigations of the theory surrounding the problem and issues observed are made in order to collect information and to get a better understanding of the area. When essential questions have been addressed and background information obtained, a hypothesis relating to the observations can be constructed.

An optimization model, based on the hypothesis, can then be developed and, thus, be used in order to test the hypothesis. To be able to quantify the studied problem, it is normally first simplified to only include the essential components. For complex problems, it is sometimes impossible to include all aspects because that can make the model too difficult to solve. When a model is developed, a suitable solution strategy is selected in order to solve the problem. It is important to choose an appropriate solution strategy depending on the type of problem to be solved, the input data and if a method is known to be good for solving the certain type of problem. Different solution methods are suitable for different types of models. First, it is important to formulate the problem and then decide the most appropriate solution method to solve it.

When the problem has been solved, the hypothesis can be evaluated by different tests and experiments. To finally draw conclusions from the results, the results must carefully be 
analyzed. When analyzing the results, the simplifications made when quantifying the problem must be considered, in addition to the quality of the data that was used for solving the problem.

When using optimization models for solving problems, the results obtained from the solutions may not always be practically applicable, or they can affect the people involved negatively (Lundgren et al. 2010). If that is the case, the negative impact may be considered by reformulating the model and then new recommendations can be developed. To find satisfactory results, usually the explained process must be revised and steps must be repeated. The process is not normally as straightforward as presented in Figure 1. It is more an iterative process where the model is successively redefined until the results support or partly support the hypothesis.

How the work was performed is briefly described in the next section.

\subsubsection{Description of the Thesis's Working Process}

The process described in Figure 1 is used for the research presented in this thesis. The work has been performed in close collaboration with the fire and rescue service in Sweden. Interviews, workshops and study visits have been done to collect information and to make observations regarding the fire and rescue service area. Decisions about which issues to consider and which support tools that should be developed were made based on the collected information and the made observations. Then, hypotheses regarding how the tools should work were constructed.

After this initial phase, optimization models were formulated in order to develop the tools according to the hypothesis. To ensure that all the important components and aspects were included in the models, discussions were held continuously with the fire and rescue service during the modeling process. Solution methods to solve the models were selected in order to be suitable for the specific models. Along the modeling process, tests were also made in order to validate and test the models to ensure they worked as desired. Finally, tests and experiments were made to evaluate and analyze the developed support tools. The results and conclusions obtained from the tests and experiments are reported in this thesis.

\subsection{Contributions}

The main contributions of this thesis can be summarized as follows;

The Swedish fire and rescue service organization is investigated. It is described how the operation currently works, how it is organized as well as how it is about to evolve in the future.

* A review of previous OR Research made in order to improve the planning process and the usage of fire and rescue resources is presented.

An optimization model for the problem of strategically locating fire and rescue resources is developed. The characteristic feature with the model is that it considers 
both the time for the first responding resource as well as the time for the last responding resource. These times can be weighted differently and the model can be used to locate multiple types of resources with respect to various demands for service.

* The term preparedness is explained and indicators that affect the preparedness are defined. A measure to quantitatively determine the preparedness is defined and validated.

An optimization model for the problem of selecting the most appropriate fire and rescue resources to be dispatched to a given accident site is developed and a greedy based search method is developed for solving the resource selection problem close to optimality.

An optimization model for the problem of relocating fire and rescue resources in order to improve the preparedness is developed and a greedy randomized search procedure is developed for solving the resource relocation problem.

Three optimization based decision support tools to facilitate the operational planning and management of fire and rescue resources are developed. The developed optimization models for selecting and relocating resources are basis for the tools.

The optimized decision support tools are evaluated through experiments with fire and rescue services in Sweden. The tools are tested for various decision-making situations.

Parts of the material in this thesis have been published accordingly;

Gustafsson, A., Andersson Granberg, T. (2012) Using Variable Neighborhood Search to Locate Fire and Rescue Resources. Proceedings of ORP3, Operation Research Peripatetic Postgraduate Program. Linz, Austria, July 16 - 20, 2012.

Gustafsson, A., Andersson Granberg, T. (2012) Dynamic Planning of Fire and Rescue Services. Proceedings of ISCRAM, the 9th International Conference on Information Systems for Crisis Response and Management. Vancouver, Canada, April 22 - 25, 2012.

The following part in the thesis has been submitted to a journal for publication,

Ulander, A., Granlund, R., Lundberg, J., Andersson Granberg, T. (2014) Preparedness Calculations to Facilitate the Planning of Fire and Rescue Resources. Submitted to Journal of the Operational Research Society (JORS). 
Most of the contents of the thesis have also been presented by the author accordingly;

Locating Emergency Resources, Presented at NOS4, the 4th Nordic Optimization Symposium. Århus, Denmark, September 30 - October 2, 2010.

Fire and Rescue Resource Location for Räddningstjänsten Östra Götaland, Poster Session at TAMSEC, the first National Symposium on Technology and Methodology for Security and Crisis Management. Linköping, Sweden, October 27 - 28, 2010.

Using Variable Neighborhood Search to Locate Fire and Rescue Resources for Räddningstjänsten Östra Götaland, Presented at EU/Meeting, Workshop on Client-centered Logistics and International Aid. Vienna, Austria, February 21 - 22, 2011.

Dynamic Planning of Fire and Rescue Services, Poster Session at TAMSEC, the second National Symposium on Technology and Methodology for Security and Crisis Management. Linköping, Sweden, October 19 - 20, 2011.

Dynamic Planning of Fire and Rescue Services, Presented at ISCRAM, the 9th International Conference on Information Systems for Crisis Response and Management. Vancouver, Canada, April 22 - 25, 2012.

Dynamic Planning of Fire and Rescue Services, Presented at NOS5, the 5th Nordic Optimization Symposium, Trondheim, Norway, June 7 - 9, 2012.

Using Variable Neighborhood Search to Locate Fire and Rescue Resources, Presented at ORP3 - Operation Research Peripatetic Postgraduate Program, A EURO conference for young OR researchers. Linz, Austria, July 16 - 20, 2012.

Planning of Fire and Rescue Resources, Presented at ISCRAM Summer School on Humanitarian Information Management, Tilburg, the Netherlands, August 15 - 24, 2012.

Preparedness Calculations to Facilitate the Planning of Fire and Rescue Resources, Presented at SOAK/NOS6, Göteborg, Sweden, October 24 - 26, 2013.

\subsection{Outline}

This thesis is structured as follows;

In Chapter 2, the Swedish fire and rescue service operation is described. It explains the operations of the fire and rescue service, its current structure, composition, and organization. It is also discussed how the operation is about to change and evolve.

In Chapter 3, the survey of related operations research work made within the subject area of planning and managing fire and rescue services is presented. The survey focuses on resource management problems and is structured according to the problems' decision levels, i.e. whether it is a strategic, tactical and operational problem as well as if the problem has a static or a dynamic nature. 
In Chapter 4, the developed optimization model for locating fire and rescue resources is presented and described. The model suggests locations, in a given area, for a given number of different types of response units (i.e. firefighters and fire vehicles) with respect to expected accidents in the area. The model is solved for different scenarios in a test area and the solution method and the obtained results are analyzed and discussed in the chapter.

Chapter 5 clarifies the term preparedness and how the fire and rescue service estimates the preparedness. The indicators for estimating the preparedness are defined and the measure to quantitatively calculate the preparedness is presented. The developed optimization model for selecting the most appropriate resources for dispatch to a given type of accident, in order to calculate the preparedness, is also presented and described. The heuristic approach for solving the resource selection problem is presented and the measure and the model are calibrated and validated for a test setting.

In Chapter 6, the optimization model for relocating resources is presented. Also, the greedy randomized search procedure that is developed and used for solving the relocation problem is described. Solutions are produced for a number of test scenarios, which are presented, and then it is discussed how the fire and rescue service may use the suggestions for relocating resources.

In Chapter 7, the three optimization based decision support tools are described and illustrated. The formulated models in Chapters 5 and 6 are the bases for the developed tools, which are developed in order to facilitate dynamic planning of fire and rescue resources. The chapter shows and explains how the tools can be practically used. Experiments are performed with thirteen fire and rescue services in Sweden in order to test the tools. These are described and the main results and conclusions from the experiments are discussed.

In Chapter 8, the main conclusions from this thesis are presented and discussed together with some directions for future research. 


\section{Fire and Rescue Service in Sweden}

This chapter discusses fire and rescue service operations in Sweden. It explains the operations of the fire and rescue service, its current structure, composition, and organization as well as the procedure for how the fire and rescue service responds to various alarms. The working approach within the fire and rescue service has, however, recently started to change in order to improve the service quality and efficiency in terms of its operations. The operational changes that are about to be introduced are described herein. In addition, the potential influence of these changes on the fire and rescue service operations in general is discussed in detail.

Most of the underlying information in this chapter is based on eight semi-structured interviews performed with seven fire and rescue services in Sweden. The seven fire and rescue services were selected according to their working approach and recommendations from Myndigheten för Samhällsskydd och Beredskap (MSB), (the Swedish Civil Contingencies Agency). The interviews were conducted to determine how the fire and rescue services in Sweden work today and to find out more about the work regarding the operational changes that are about to be implemented across the fire and rescue service organizations in Sweden. The two or three fire and rescue service personnel who were interviewed each time consisted of fire officers, internal commanders or incident commanders.

\subsection{Operational Structure}

In Sweden, the municipalities are responsible for providing an efficient system for responding to accidents on a local level. The legislation that regulates the fire and rescue service operation in Sweden is called the law on protection against accidents - Lagen om skydd mot olyckor (LSO, 2003:778). According to the LSO, fire and rescue service encompasses the rescue operations that the government or the municipalities are responsible for, in cases of accidents or when there is an imminent risk of accidents, in order to prevent or limit the damage to people, property and the environment. The fire and rescue service should be planned and organized so that they can begin emergency work within an acceptable period of time and perform the response efficiently. The regulations in the LSO aim to ensure equivalent protection against accidents across the entire country (LSO, 2003:778). It is then up to each municipality to develop its own organization for fire and rescue service based on local conditions.

Sweden is divided into 290 municipalities of different size and each with a different population. Each municipality is responsible for the fire and rescue service in its own geographical area (Räddningsverket, 2008). The fire and rescue services within each municipality are then responsible for satisfying the municipality's objectives of safety and security. The goal is that all citizens should have a safe and secure environment and that the risks for fires and other accidents should continually decrease (Björnberg and Melin, 2003). This means that the fire and rescue service must support the municipality with an efficient emergency protection plan within its boundaries. The county administrative board is then responsible for ensuring that the municipalities operate a competently well-staff and fully resourced fire and rescue service (Räddningsverket, 2008). 
The demand for fire and rescue service usually arises from various accidents, and it may vary with time and space and also from one municipality to another. The demand normally varies even between different areas within one municipality. One area can, for example, be more affected by accidents than other areas, and the demand may vary during the day or year at different locations and areas in the municipality. To be able to execute an efficient emergency response to urgent alarms in all areas in a municipality, the neighboring municipalities usually collaborate across the border. Such collaboration also makes the fire and rescue service operation more robust for areas along the municipal boundaries, which can otherwise sometimes be problematic to handle. For example, if there is an urgent accident close to the municipal boundary and the fire and rescue resources that are closest to the accident site are located in the neighboring municipality, it might then be more efficient to dispatch the resources from the neighboring municipality to the specified accident site.

Several municipalities can also form a regional alliance and then collaborate within their areas. The municipalities are in that case collectively responsible for managing fire and rescue service in all municipalities that belong to the alliance (Räddningsverket, 2008). Regionally controlled fire and rescue services in Sweden haven become increasingly common, i.e. to develop regional collaborations and merge several fire and rescue services into regional association. The main purpose of forming regional alliances is to make the fire and rescue service operations more efficient and to cut down on costs (Rosenberg, 2001). Another ambition is to coordinate more powerful rescue teams and streamline the preventative activities. An advantage is the possibilities of joint development of knowledge for risk analysis as well as developed knowledge of effective preventative actions. It is also a benefit for smaller municipalities or organizations with limited resources in their access to better opportunities to perform safety improvements, e.g. to perform risk analysis. A disadvantage with regionalization and the forming of alliances may be that the knowledge of the local risk situation sometimes decreases (Rosenberg, 2001). If the knowledge decreases, it could, for example, cause a decrease in the quality of accident prevention work. Fire and rescue personnel with knowledge of the local environment are necessary in order to adapt the operation to local circumstances.

Over the last several years, many municipalities in Sweden have cooperated across regions and formed alliances by combining several fire and rescue services from different municipalities. Mostly, alliances are formed by municipalities with larger cities or municipalities with high population density. The formed alliances are, however, not always permanent, which means they can be reorganized over the years. In 2000, there were 234 municipal organizations for fire and rescue service, of which 22 consisted of alliances where several municipalities collaborated in order to coordinate and manage emergency service (Departementsserien, 2009). At the beginning of 2006, there were 30 alliances in which several municipalities collaborated together, and altogether there were 212 fire and rescue service organizations (Persson, 2006). By 2010, this had changed to 185 fire and rescue service organizations, of which 35 consisted of alliances with several municipalities (MSB, 2011). In 2013, there were 36 fire and rescue service alliances including several municipalities and 184 fire and rescue service organizations (Sveriges Kommuner och 
Landsting, 2013). An overview of the recent changes in the numbers of organizations and alliances within the Swedish fire and rescue service is presented in Figure 2.

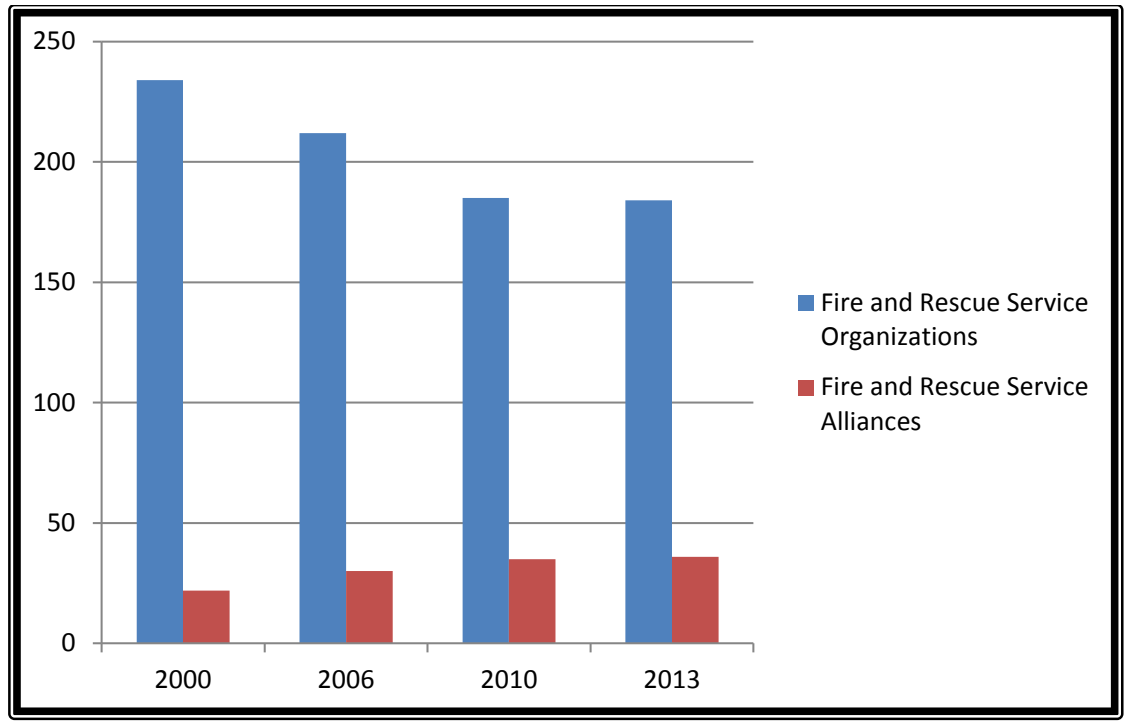

Figure 2. An overview of the number of fire and rescue service organizations and alliances over the last few years

In cases of larger and more complicated emergencies or when several municipalities are affected by a major emergency incident, the county administrative board is normally responsible for the emergency services in those municipalities. The county administrative board shall also provide the municipalities with advice and information to support their operations (Räddningsverket, 2008). Sometimes, it may also be necessary for the government to take over the responsibility for the emergency service in large and complicated situations. The government is always responsible for emergency service associated with emissions of radioactive substances, environmental emergencies at sea, maritime emergency service, aeronautical emergency service, alpine emergency service and tracing of missing people (Räddningsverket, 2008). The government and the municipalities must also coordinate activities and collaborate with each other and other authorities to efficiently provide a good fire and rescue service.

The municipal fire and rescue service organizations usually differ in structure, also in how they operate and work in their organizations, as well as how they manage their responses on a daily basis. It may include differences in management structure, information and communication systems, staffing requirements for different tasks or how different activities are performed. For example, there is a major difference between how the work is conducted in a large organization with full-time firefighters compared to a smaller organization with parttime firefighters. Organizations may also differ depending on if the municipality is highly populated or consists of rural areas, if there are larger urban areas in the municipality, depending on the municipalities' environment, or if there are high-risk zones/objects in the area such as industries, factories, public buildings, ports, airports, etc. 


\subsection{Required Support}

The need for assistance from the fire and rescue service may vary with time, but depends also on the demography, the geography and the infrastructure. The fire and rescue service responds to both urgent and non-urgent alarms. Usually, the need arises from various types of accidents. In Sweden, the most common reason for a fire and rescue service response is due to automatic alarms, which represent almost $37 \%$ of all the fire and rescue service responses made in Sweden in 2012 (MSB, 2013). However, frequent accidents in Sweden that cause most of the serious injuries and loss of life are building fires (11\%) and traffic accidents (19\%). These accidents collectively represented $30 \%$ of all the rescue efforts made in 2012 (MSB, 2013). Other common reasons for fire and rescue service responses are fire accidents (not in buildings), which comprised $13 \%$ of all the fire and rescue service responses during 2012, and false fire alarms, which comprised around $8 \%$ of all the fire and rescue service responses during 2012 (MSB, 2013).

There are also other events and situations that initiate fire and rescue operations. The fire and rescue services are, for example, also needed for preventative work, inspections, official visits, public events, various educational programs and other activities in order to ensure public safety. The latter activities are requirements that can usually be planned in advance, in contrast to urgent accidents, which are not planned. In cases of urgent accidents, it is important for the fire and rescue service to arrive at the accident site as quickly as possible and deploy the suitable resources. Depending on the type of accident and the situation, different resources and different number of resources are requested at the accident site in order to perform an efficient response. If there is a building fire, the need for resources depends, for example, on the type of building that is under fire. If the building is a dwelling such as a house, the need for resources is probably not as extensive as if the fire were in an industrial facility with dangerous substances. Also, the need for resources is not likely the same for a traffic accident as for a building fire. The magnitude of the accident is something that also affects the type and volume of resources. A traffic accident with a single vehicle probably does not need as many or the same types of resources as a traffic accident with several vehicles involved. The need for resources will probably also differ depending on if dangerous goods are involved or not, or if hazardous chemicals are leaking. Special units can, thus, be needed for certain emergencies. In order to identify which and how many resources are needed for an incident, accidents are usually divided into different groups depending on the type of accident and various factors such as these just discussed. This is further explained in Section 2.5

\subsection{Planning and Resource Management}

By tradition, the Swedish fire and rescue service has been characterized by relative static planning and resource management. The resources (firefighters and fire vehicles) are normally located at fire stations, which are predominantly strategically located in populated areas such as larger cities and urban areas. Firefighters normally work in shifts and operate in teams composed of a number of personnel with different skills and competencies. A fire and rescue team is traditionally composed of the same team during an entire working shift. However, the number of firefighters included in a team and how the shifts are structured 
varies between the fire and rescue services. How the fire and rescue services are organized and composed depends, for example, on the size of the fire and rescue service, local conditions and variations, regulations as well as political decisions.

When an emergency arises, resources are dispatched to the site. The accident site partly predetermines from which fire station resources should be dispatched. But, how many and what type of resources to initially dispatch to an incident typically depend on the local alarm plans; see Section 2.5. For example, a fire and rescue service may send four firefighters to a particular type of incident while another may send five or six firefighters to the same type of incident. If many resources in an area are busy, it is also usually partly predetermined how the remaining available resources from other fire stations should be utilized and if and how they should be relocated to maintain an adequate preparedness to new accidents. However, the strategies and regulations regarding how resources should be utilized and organized vary between the fire and rescue services. Often, these decisions are contingent on local conditions, resource availability, weather variations, historical approaches and former decisions that have survived.

When the rescue work is concluded, the firefighters normally return to their home station. If additional resources have been relocated due to an incident, these resources also normally return to their home (normal) locations when the rescue work is finished. The firefighters are then again ready for new calls and other missions.

\subsection{Resources and Tactical Units}

Within the fire and rescue service, there are many different types of resources. The most wellknown resources are probably firefighters and fire vehicles. The firefighters can have various experiences, competencies and skills, e.g. ability to use special tools or equipment, management of certain vehicles or supervision of fire and rescue teams. There are many kinds of vehicles in the fire and rescue service, such as fire engines, ladder vehicles, small vehicles, tank vehicles and cross-country vehicles. Various types of firefighters and fire vehicles are needed for different types of tasks, and they can be used for different purposes depending on the situation and type of accident. In addition to different types of vehicles, firefighters, fire officers and other personnel, the fire and rescue services also have various equipment and tools to manage firefighting and other incidents and activities. This may involve, for example, pumps, hoses, hydraulic cutting tools and floating boards for saving lives in water or on ice (Björnberg and Melin, 2003). In each municipality, there should always be a chief fire officer on duty (Räddningstjänsten Storgöteborg, 2011). The chief fire officer has the overall responsibility for the operation and ensures that activities are appropriately arranged in relation to the objectives set forth in the legislation and in the municipal action program (Räddningsverket, 2008). Titles and names on resources (on vehicles and persons) can vary between the municipalities.

In municipalities where the risk for accidents is high or where the volume of emergency calls is high, there are usually fire and rescue teams with full-time firefighters on duty at all hours, both day and night. In Sweden, there are around 150 municipalities with full-time firefighters (Olofsson, 2013). Usually, the municipalities with cities or larger towns, i.e. municipalities 
that are quite highly populated, are those with full-time fire services. In less populated municipalities with few emergency calls, fire and rescue services may consist of part-time firefighters and fire officers who are available when required. In many of these smaller organizations, there are often full-time fire officers and part-time firefighters. Part-time firefighters normally have a second job, but when on duty, they must always be available. When part-time firefighters are called out, they will be alerted and the response normally goes via the fire station, i.e. they first make a stopover at the fire station and from there they continue to the accident site. The preparation time for part-time firefighters is normally five minutes; 90 seconds for full-time firefighters. That means the firefighters have to leave the station within 90 or 300 seconds from the time they receive the alarm. In some places, there are so-called volunteer firefighters involved in rescue operations, for instance on smaller islands or in rural areas. The role of volunteer firefighters is very similar to that of part-time firefighters, but they are not on duty like their part-time counterparts and they are not required to respond to alarms. Volunteer firefighters may respond to an emergency if they are in the neighborhood and are available. They only respond to accidents on their own accord.

Other personnel resources included in fire and rescue service operations are daytime fire and rescue service staff. The daytime staff consists of fire and rescue personnel who, for example, can perform preventative work such as inspections and controls, give courses and education, perform life-saving and basic emergency responses such as perform cardiopulmonary resuscitation (CPR) (Räddningstjänsten Östra Götaland 2011:1). Daytime fïre and rescue service personnel are not firefighters and cannot replace them, but serve as a valuable complement to the fire services operation.

Resources and equipment should be adapted to the general needs of each municipality (Räddningsverket, 2008). The composition of resources for each municipality is, however, contingent on political decisions (Räddningstjänsten Storgöteborg, 2011). Larger organizations usually provide more and different types of resources and equipment, such as various expertise and special units, while smaller organizations usually provide basic equipment and fewer resources.

When the fire and rescue service responds to incidents or other missions, the resources normally form various tactical units. Tactical units consist of different vehicles, personnel and equipment. Resources are normally combined into tactical units because it is, from a management perspective, more convenient to handle compared to individual resources (Svensson et al., 2005). To coordinate an effective response to an accident, Björnberg and Melin (2003) hold that it is essential that the tactical units consist of adequate vehicles with useful equipment and that they are staffed with personnel with the right expertise. If the unit lacks any of the components, the resources will not be utilized effectively in conducting a response at the accident site, and the undesirable consequences of the incident may then increase. If, for example, a cutting tool is required for a traffic accident and it is absent, the entire rescue may suffer. 
It is possible to use smaller tactical units that are operated by one person to larger units operated by, for example, fifteen persons. If needed, the rescue team at an accident site can always be extended by additional resources and units.

In Sweden, there are a number of predefined tactical units that are composed of resources that are usually required for common types of accidents. Examples of such tactical units are rescue units, ladder units, water supply units, command units, first response units and special units (Räddningstjänsten Jönköping, 2011). Special units include some form of expertise and/or additional equipment compared to the standard complement of rescue resources. Special units can also consist of combinations of several units. Special expertise or special tools may, for example, include infrared cameras, chemical handling equipment, surface rescuers, penetrating tools, foam extinguishers and tools for handling gas tanks (Björnberg and Melin, 2003).

The tactical units in Sweden are currently dominated by rescue units consisting of one basic fire vehicle, four firefighters and one fire officer. Such a unit is almost always dispatched to an accident site. The rescue unit provides all the basic equipment for firefighting and rescue service such as leadership personnel, water supply and BA (Breathing Apparatus) rescue. BA firefighters have special equipment, such as breathing apparatus, to be able to work in smokefilled spaces.

The tactical rescue unit with five persons is probably the most common since the staffing is controlled by the current regulations for BA rescue. According to the Swedish legislation regarding staffing of BA operations, at least one fire officer and three BA firefighters are required, of which one must be able to supervise the BA crew (AFS, 2007:7). In order to ensure firefighters' safety, they must also have access to proper water supply during the entire rescue operation. Therefore, one more firefighter is usually needed. However, if it is possible to use technical solutions to guarantee safe access to water supply, such a solution may possibly replace one of the firefighters.

\subsection{Alarming}

A fire and rescue service in Sweden is usually alerted via an emergency center run by the company SOS Alarm Sweden AB. An alarm is normally generated from an incoming call. It can, however, also arise automatically, and in that case the alarm goes directly to a predefined fire station depending on where the alarm was initiated. Automatic alarms usually arise from, for example, public buildings, hospitals, hotels, schools and industries (Räddningsverket, 2008).

If a fire and rescue service alarm arises from an incoming call, the call is always transmitted to SOS Alarm, which operates the emergency number 112 in Sweden. Most emergency calls are also handled by SOS Alarm, i.e. they answer the call and dispatch resources to emergency sites. A fire and rescue service alarm can, however, also be handled by a dispatcher at a fire and rescue service (Räddningstjänsten Storgöteborg, 2011). How it works depends on which part in Sweden the incoming call originates from. If the call is handled by a fire and rescue service, the call will be forwarded by SOS Alarm to the dispatcher at the fire and rescue 
service. A fire and rescue service alarm can, thus, be handled differently, and there is currently no standardization of the alarming process.

Depending on where an accident occurs geographically, the fire station that should be alerted is normally predetermined. Generally, it is the closest station to the accident site (Södertörns Brandförsvarförbund, 2011). Initially, units located at the alerted fire station are dispatched to the accident site, usually by either an operator at SOS Alarm or a dispatcher at a fire and rescue service.

Which resources should be dispatched to an accident are usually based on the information received from the incoming call or, alternatively, on the incoming information from an automatically received alarm. It might be several people involved in the decision making about which resources to dispatch to a specific accident. It is, however, always a chief fire officer at each fire and rescue service organization who is ultimately responsible for the allocation of resources (Räddningstjänsten Storgöteborg, 2011). It is also the chief fire officer who decides which alarms the fire and rescue service should respond to. To facilitate the initial dispatching of resources, alarm plans may be developed in order to assist in such decisions. Alarm plans give the decision-makers predefined suggestions of what kinds of resources should initially be sent to a particular type of accident. When developing alarm plans for specific accidents, the accident's magnitude, urgency, expected development process, and estimated consequences are considered (Björnberg and Melin, 2003).

In Östergötland County, the alarm plan for a traffic accident consists, for example, of four firefighters, one fire officer and one basic fire vehicle (Räddningstjänsten Östra Götaland, 2011:3). Also, a special cutting tool to assist in the release of trapped and injured victims is normally included in the alarm plan for traffic accidents. Similarly, for a building fire, the alarm plan established in Östergötland County includes four firefighters, one fire officer and one basic fire engine. The alarm plan for building fires must then also ensure that there are enough firefighters with BA (breathing apparatus) skills to be able to perform rescue operations in smoke-filled spaces. If there is a fire in a high-rise building, the alarm plan must also include a ladder unit (Räddningstjänsten Östra Götaland, 2011:3).

Alarm plans are developed on a local level, which means that they can vary between the organizations and the municipalities in Sweden. It is not necessary to have alarm plans, so there might be municipalities that do not use any alarm plans. If municipalities do have alarm plans, they are normally established by the chief fire officer at the local fire and rescue service, who is also responsible for the allocation of resources for the fire and rescue service. Alarm plans do not exist for every type of accident, and every possible accident cannot be classified as a particular type of accident. This means that specific judgment from decisionmakers may also be needed when dispatching resources to different emergencies.

If an organization does have alarm plans for a certain accident type, the resources specified in the corresponding alarm plan must always be dispatched to the accident site initially as the minimum quantity of resources (Räddningstjänsten Storgöteborg, 2011). The operator at SOS Alarm, the dispatcher at the fire and rescue service or the chief fire officer can always dispatch additional resources to the accident site as needed. It is, however, only the chief fire 
officer who can recall resources from an accident site if, for example, too many resources have been dispatched or if resources are no longer needed.

\subsection{Response Time and Response Capability}

Response time may be one of the most important factors in survivability of an accident. To reduce suffering and negative consequences from an accident, the faster the response, the better. Undesirable consequences from an incident might increase if the time between notification of the emergency and actual response is protracted. The severity of negative consequences may also increase if there are too few resources to conduct an efficient response at the accident site. For example, a fire has more time to spread while an emergency response is being coordinated, or if any significant resource for extinguishing the fire is missing. Damages and injuries due traffic accidents may also increase with protracted response time. A neck injury can, for example, be exacerbated before treatment if the response time is extended. Traffic accidents may also sometimes cause danger to other passengers. They may, for example, obstruct road accessibility, and in the worst case also cause more people to become injured or in some way involved in the accident. There are also types of incidents where the consequences do not become worse with longer response times, although these types of incidents are quite rare (Andersson et al., 2005). One example may be if there is a traffic accident involving one vehicle where the driver does not become injured at all. Another example may be if the driver dies immediately, and where the car in which the victim was travelling does not cause any traffic congestion.

The response time is the time from the point an alarm is received by a rescue unit, until the firefighters can start working at the accident site. The response time includes preparation time, travel time and intervention time (Andersson et al., 2005); see Figure 3.

The preparation time is the time from when an alarm is received by a unit until the unit is on its way to the incident site; the travel time is the transportation time for travelling from the unit's current position to the incident site; and the intervention time is the time from when the unit has arrived at the accident site until the rescue work can begin. The response time is then directly related to the unit's current position, the unit's speed and the firefighters' preparation time. 


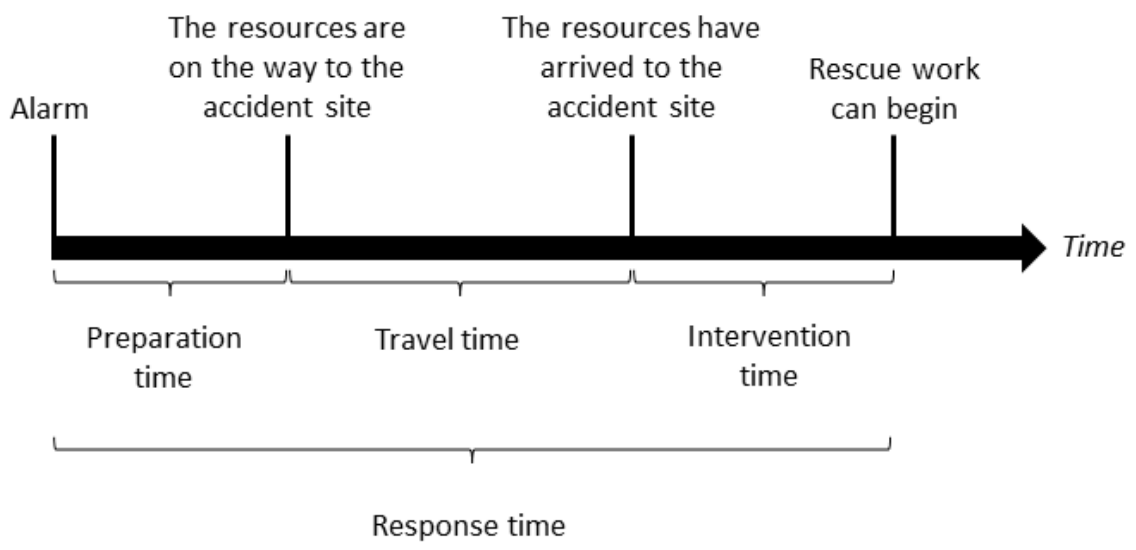

Figure 3. Illustration of response time.

The response time is also related to response capability, which is the ability of the deployed emergency personnel and resources to manage the accident site. As previously discussed, it is important that the right type and the right amount of resources arrive at the accident site in order to conduct an efficient response. Sometimes it is not possible to start the response work as intended before all the requested resources have arrived at the accident site. Some resources can, for example, only work efficiently together with other specified resources. The equipment of a standard fire engine is, for example, needed in order to utilize a tank unit's entire capacity (Räddningstjänsten Kinda Kommun, 2011). Therefore, the response capability may deteriorate if some or all of the requested resources' response time increases, since the firefighters might not be able to work efficiently until all the required resources have arrived. The correlation between accidents, response time and response capability is presented in Figure 4. 


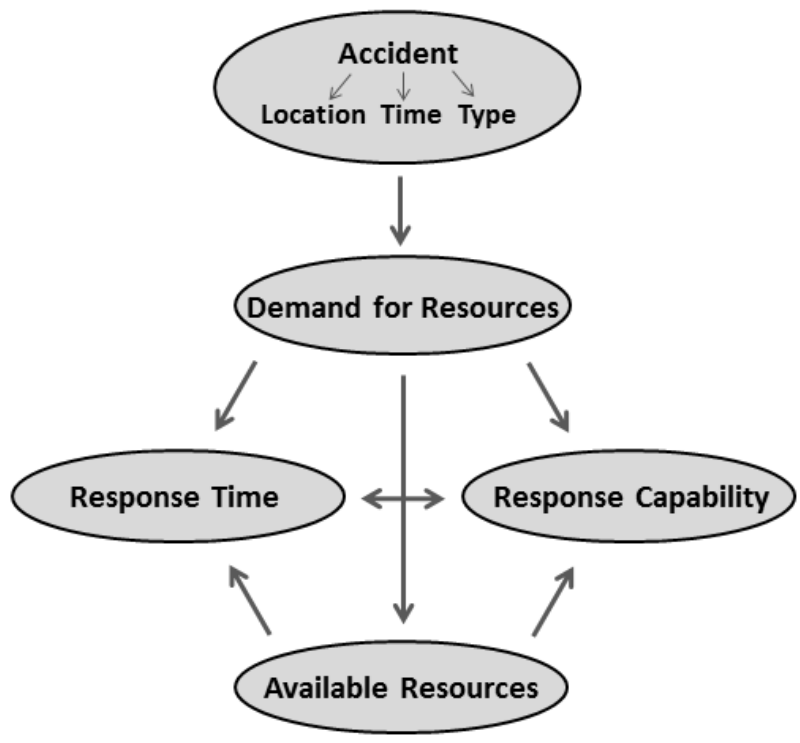

Figure 4. The correlation between accident, response time and response capability.

Both response time and the response capability are related to the demand for resources as well as the current available resources. Response capability usually decreases if the demand for resources increases, since more resources will then become utilized, i.e. the available resources decrease. In these circumstances, the response time typically increases. The demand for resources usually depends on the type of accident and on when and where the accident occurs. In the planning process for fire and rescue services, it is thus very important to strive for the most efficient usage of the resources in order to always reach incidents within a reasonable period of time.

\subsection{Operational Changes}

Working methods and procedures within the fire and rescue service in Sweden have recently started to change in order to improve emergency response service quality and efficiency. As the demand for fire and rescue service evolves, changes to routines and working methods are also sometimes necessary in order to manage all types of needs and ideally prevent and mitigate the consequences of emergencies.

Statistical analysis shows, for example, that accidents are more likely to occur in such places where accidents have previously occurred, i.e. mostly on high-traffic roads, in cities, villages and other areas where people can be found (see; for example, Martinsson, 2013). Therefore, fire stations are normally located in cities and urban areas (close to the population). But, the statistical analysis also shows that emergency response needs vary with time and changes during the day and with season. It is, therefore, important for the fire and rescue service to adapt the rescue service to the same pattern in order to ensure its availability as and where needed (Räddningstjänsten, Jönköping 2011). 
It has also been shown that the incidence of less severe accidents and fires is relatively high in comparison to more extensive accidents and fires (Björnberg and Melin, 2003). It may, thus, not always be necessary to send a full rescue team of, for example, five firefighters to an accident. Sometimes two or three firefighters may be enough, such as on an emergency response to extinguish a fire in a litter bin. The fact that the incidence of less severe accidents and fires is higher may also be due to the increasing focus that has been placed on the initial phase of an accident. If accidents and fires can be resolved within a few minutes, before a flashover or a critical condition occurs, the negative effects can usually be reduced. An essential challenge is, thus, to be able to begin the rescue work as early as possible by dispatching the closest available and most suitable resources (Räddningstjänsten Jönköping, 2011).

The importance of coordinating and dispatching fire and rescue resources in relation to current demand and current risk situation has forced the working approach to become more dynamic in order to increase the flexibility and adaptability of emergency operations with respect to current needs. Accordingly, the trend in the fire and rescue services in Sweden seems to be moving towards the usage of more and smaller tactical units, i.e. units that consist of individual firefighters or groups of two to three firefighters. The smaller units would likely be more widespread, but they can be merged and form rescue teams in any combination at an accident site (Räddningstjänsten Storgöteborg, 2011). Resources can, thus, be dispatched from different places and fire stations depending on specific demands at an accident site. In this way, the currently available resources that are needed at and most suitable to a particular accident can be dispatched (Södertörns Brandförsvarförbund, 2011).

In order to utilize smaller units that are also more widespread in the most efficient way, the alarm process would be modified and adapted to the structural changes. Today, fire and rescue service alarms are still normally received by the fire stations, and then resources are in turn dispatched from the stations to the accident site (Södertörns Brandförsvarförbund, 2011). Since the smaller units would not typically be located at fire stations, it would be more beneficial if resources could directly receive the alarm and be dispatched to an accident and not necessarily via a fire station. This would also likely reduce response time since the closest and most suitable resources could be dispatched directly.

The assistance given by the personnel at SOS Alarm is also something that is about to be developed and improved for fire and rescue service operations. With better assistance from the personnel at the emergency service center, it should be easier to take advantage of each individual's capability (at the accident site) to take action in specific situations. The personnel should be able to provide the person who initiated the call with more targeted instructions and advice for the specific situation (Björnberg and Melin, 2003, SOS Räddningscentral Norrköping, 2011).

Figure 5 presents simplified overviews of how the fire and rescue services in Sweden mostly work today and how they are expected to change when introducing the new aforementioned working methods and procedures. The pictures show how resources (tactical units) are available in an area and how a 112 emergency service center (SOS Alarm or a dispatcher at a 
fire and rescue service) can initiate the deployment of the resources. The latter is indicated by the arrows in the pictures. The picture to the left shows the current structure, the way in which the resources work together in teams, and that they are usually located at fire stations. Alarms are typically initiated by an incoming call from someone at the accident site and the resources are alerted via a fire station, which is indicated by the arrows. The picture to the right shows that the resources are divided into smaller crews and that they are more spread out strategically. The resources can directly and individually (as well as via a fire station) be dispatched to an accident by the emergency center. The two-way arrow between the accident site and the emergency center indicates that the dispatcher at SOS Alarm should also be able to give advice and better assistance to the person who initiated the call depending on the accident and the specific situation.
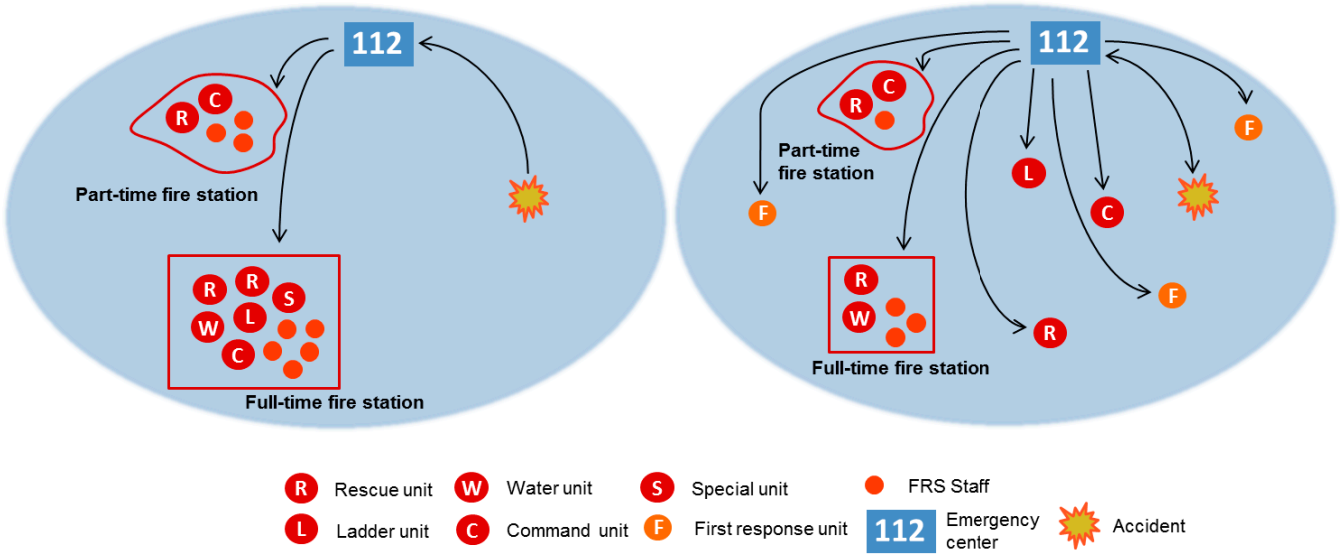

R Rescue unit
L Ladder unit

S Special unit

First response unit

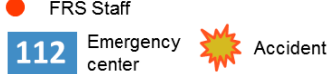

Figure 5. An illustration of how the Swedish fire and rescue service works today (to the left) and how it is expected to change when new working methods are implemented (to the right).

To meet the challenge of taking care of accidents in an early stage, so-called "first responders" have been introduced in some fire and rescue services, and they have become important and also a key asset for the fire and rescue service. A first responder is the first resource to arrive at an accident site. This person is a special first response person who is trained to take care of emergencies in the initial phase. First responders can, however, be anyone, e.g. firefighters, fire officers or daytime fire and rescue service personnel. A first responder can also for example be medical personnel, security guards or home care personnel with first response competence (Weinholt and Andersson Granberg, 2013). First responders provide basic equipment to be able to perform basic rescue work and lifesaving care. Since there almost always are important tasks that can be performed by one or a few persons in the initial phase of an accident, even in complicated situations, the quick arrival of first responders has been shown to be beneficial. First responders can, for example, perform essential preparatory work while waiting for the ordinary rescue team to arrive at the accident site. Such tasks may include investigation of the accident site and the surrounding area, performance of cardiopulmonary resuscitation (CPR), submission of status reports to 
upcoming firefighters, initiation of the rescue effort, extinguishing of small fires and support of the injured (Weinholt and Andersson Granberg, 2013). First responders may sometimes also assist in the rescue work after the ordinary rescue team has arrived at the site. For example, they can provide support with equipment and help to secure the accident site (Björnberg and Melin, 2003). Depending on the competencies and the training of the first responders, they may in some cases also be members of the ordinary rescue team formed at the accident site (Räddningstjänsten Jönköping, 2011). If the first responder is a firefighter, they are normally always included in the ordinary rescue team.

Another thing has also been recently recognized as important and introduced in some fire and rescue services is responding to emergency medical alarms in situations when it may take some time before an ambulance could be dispatched, i.e. waiting for the ambulance (i väntan på ambulans - IVPA) alarms. When personnel from the fire and rescue service respond to IVPA alarms, they perform basic pre-hospital work before healthcare personnel arrive at the accident site. Responding to IVPA alarms by the fire and rescue service has increased recently (Räddningstjänsten Östra Götaland 2011:3).

Something that would be beneficial for the fire and rescue service, which the fire and rescue service staff who were interviewed during this study also think might be changed in the future, is if part-time firefighters could be sometimes dispatched directly to an accident site instead of making a stopover at the fire station. This would be particularly beneficial if the part-time firefighter is already close to the accident site. This will probably have a positive effect on the response time and then also on the initial rescue work during an emergency response. The reason why part-time firefighters still normally make a stopover at the fire station when they are alerted is probably due to traditions and historical decisions. The firefighters are used to forming a rescue team at the station and then travelling together to an accident site.

The operational changes that have started to be introduced in order to improve fire and rescue service quality and efficiency are summarized in Table 1. 
Table 1. A summary of operational changes.

\begin{tabular}{ll}
\hline Operational Changes & Explanation \\
\hline \hline Using smaller tactical units & $\begin{array}{l}\text { Firefighters are working } \\
\text { individually or in groups of two } \\
\text { or three firefighters. Several } \\
\text { smaller units can converge and } \\
\text { form a rescue team at accident } \\
\text { sites. }\end{array}$ \\
\hline
\end{tabular}

Directly and individually dispatching of resources

Better assistance from the emergency service center

Using first responders

Responding to IVPA (waiting for ambulance) alarms

Directly dispatching of part-time firefighters
Resources can be alerted and dispatched directly to accidents, and not necessarily through or via a fire station.

The personnel at emergency service centers should be able to better support the person who initiated the call with more targeted instructions and advice for the specific situation.

Special first responders can make quick first responses to accidents. The persons are trained to take care of emergencies in the initial phase.

Fire and rescue service personnel can respond to emergency medical alarms when it may take some time before an ambulance could be dispatched.

Part-time firefighters can be dispatched directly to an accident site without making a stopover at a fire station.

\section{Benefits}

Firefighters at various

locations reduce the response time and, thus, also increase the coverage and the preparedness level. It might also improve work during the initial phase of an accident.

The closest and most suitable resources can likely be dispatched to an accident. Also, it might improve the response time and emergency work during the initial phase of an accident.

It should be easier to take advantage of people at the accident site. They may likely be able to take action while waiting for the fire and rescue service.

First responders can likely perform essential preparation work, basic rescue work and lifesaving care before the ordinary rescue team arrives.

Fire and rescue service personnel can perform basic pre-hospital work and lifesaving care before healthcare personnel arrive.

Improved usage of part-time firefighters. It might have positive effects on the response time and the emergency work during the initial phase of an accident.

The operational changes, summarized in Table 1, have already started to be introduced in some fire and rescue service in Sweden. Some organizations are more interested in introducing such changes than others. The process of adaptation to the changes also varies between the different fire and rescue services. Since the fire and rescue services in Sweden differ quite significantly both in composition and in operation, all the changes might not be appropriate for all types of organizations. Which fire and rescue service organizations that have started to change their operation and what kind of changes they have introduced are not examined in this thesis. This information is worthy of further interrogation to establish a clearer picture of the fire and rescue services in Sweden. 


\subsection{How the Operational Changes Influence the Fire and Rescue Service}

The main differences that arise from the operational changes described in the previous section are that firefighters will likely be more widespread in an area, both individually and in smaller groups, as well as the ability to be directly dispatched to accidents. This may provide better flexibility to respond to incidents. With resources positioned at multiple locations, the probability that resources are available nearby in case of an accident will increase. That means an improvement in coverage, i.e. the ability to reach a population and property in a certain period of time (Räddningstjänsten Jönköping, 2011). Introducing smaller units and first responders as well as efficient utilization of part-time firefighters may improve response time to emergency incidents.

Alerting the closest rescue team of five firefighters (which is the standard protocol of most fire and rescue services today) ensures a suitable and safe response, but not always the quickest, and it may not always provide the right competencies based on the specific needs (Björnberg and Melin, 2003). If resources are more widespread, the workforce will be more flexible and can be customized to each specific type of accident. The specific expertise that is needed could then be provided from the most suitable location. Sometimes it may, for example, be more beneficial to dispatch two firefighters from one location and others from another location in order to ensure a quick response and the right competencies of firefighters.

If the fire and rescue services use more flexible tactical units and smaller groups of firefighters compared to predefined rescue teams of, for example, four or five firefighters, one major difference is that the units usually must be able to form a team at the accident site instead of at the fire station. When a team is formed at the fire station, the firefighters included in the team usually drive together to the accident site. During the trip, the team may undertake some preparation work in order to ensure the most efficient emergency response. A disadvantage of the new approach could be that the tactical unit would not be able to undertake such preparation during the trip to the accident site. However, focus has increasingly been directed towards the initial phase of an accident, and an accident's scope and magnitude are not normally known and, therefore, hard to define until the first resource/resources have arrived at the accident site. It would, therefore, probably be better if someone could quickly reach the site, and then provide accurate information about the accident to the rest of the dispatched resources instead of trying to prepare a rather uncertain rescue effort when driving to the accident site. The interviewed fire and rescue service staff also mean that it would be better if one or a few firefighters reach an accident site early, and that the response team gradually is built up, than if all resources arrive later at the same time. Perhaps it would also be more valuable if one firefighter arrives within seven minutes, three within eight minutes, and three within twelve minutes, than if all arrive within ten minutes. When all the resources have arrived, a rescue team can be formed at the accident site. The timing of when a particular unit arrives at an accident site is critical to the emergency response operation. If a fire, for example, can be extinguished early, the undesirable consequences may not be as destructive as if the fire spread. Only a few seconds or a minute can make a great difference. 
As mentioned before, the coverage and the response time normally improve by drawing resources from multiple places. Accordingly, it is beneficial for the preparedness, i.e. the ability to respond to new incoming calls. However, since only a small portion of the fire and rescue service working tasks consist of responding to urgent calls, it is also important to consider non-urgent missions and other tasks, e.g. inspections, supervisions, education, training sessions and other important preventative work. Since such tasks can usually be performed by only one or a few firefighters, the planning of non-urgent missions also benefits due to a more flexible working approach with smaller units or combinations of units.

There are different opinions among the fire and rescue services in Sweden regarding the operational changes. Some fire and rescue services believe there are definitive advantages with the new approach while others are more uncertain. Mostly, it is the division of tactical units that still seems unfamiliar to many fire and rescue services. Currently, a rescue team is not often divided into smaller groups unless it is absolutely necessary. According to interviews with rescue staff, dividing rescue teams in smaller groups is also something that firefighters themselves mostly consider as a disadvantage. Since firefighters in Sweden normally have worked in that manner and feel comfortable by working in specific teams, it is not surprising that they are uneasy about changing working conditions.

Another central aspect is that fire and rescue resources are traditionally located at fire stations. The mentality within the Swedish fire and rescue services is still dominated by the notion that resources are not likely located or relocated to places other than fire stations. Such a routine may be difficult and time-consuming to change within the fire and rescue services. However, the way of thinking within the fire and rescue service has slowly begun to change into believing that it may not always be appropriate, in relation to the demand and the service quality, that fire and rescue resources are predominantly located at fire stations. But still, the idea is quite foreign to most of the fire and rescue service organizations.

Even if the fire and rescue services in Sweden mostly have the same traditional structure and generally handle resources in a similar manner, they mostly use working approaches according to their own organization's conditions, guidelines and activities. For example, fire and rescue services have different strategies when dispatching resources, they use different alarm plans and alarming processes, firefighters' working shifts and how they are divided in working teams varies, planning systems and software differ and also names and titles of resources and tactical units sometimes vary. When fire and rescue services have started to introduce changes into their operations, a desire has according to the interviews escalated to also implement more uniform working approaches and guidelines regarding resource planning. To change the working approach, one thing could for example be to let fire and rescue services obtain insights and deeper knowledge about other fire and rescue services' working strategies and how they work under various circumstances. The interviewed fire and rescue services believe it would be inspiring and interesting to get ideas from other organizations in order to introduce the same working approach into their own operation and organization. Also, when something works well in one organization, it is easier to believe that it probably will work within one's own organization as well. If a new working approach and new working strategies should be designed and implemented within a fire and rescue service, 
many factors and parameters must first be carefully investigated. How this should be done and how the approach and the strategies should be designed are not considered in this work. However, it might be a large process. When changing a working approach, organizations must usually be changed gradually. The working approach and the strategies must also be suitable for all types of fire and rescue service organizations, both small and large. It might take several years to change the culture within and traditionally rooted patterns in order to achieve changes in working procedures.

When introducing new working approaches or operational changes, new requirements and new situations may also arise. As fire and rescue services in Sweden have started to use smaller groups of firefighters, first responders and begun to respond to IVPA alarms, they have for example found that the planning process has become more complicated. When resources are divided in smaller groups and are more widespread, it is more challenging to ensure an adequate overview of all the resources. For example, in case of an accident, it may not always be obvious which resources to dispatch to the accident site. It is furthermore just as difficult to decide how to relocate resources, if necessary, in order to maintain adequate preparedness. In such situations, it might be essential with efficient planning tools that, for example, can support the dispatcher in order to coordinate and allocate resources. Decision support tools that can be valuable and used in various planning situations regarding resource management have been developed and are described in Chapter 7. If all fire and rescue services in Sweden implement such support tools, the fire and rescue services are more likely to use a more uniform working approach, since they all use the same support tools. By getting support from the same system, it should also for example be possible to similarly evaluate and identify the preparedness, which today is something that commonly is perceived differently by fire and rescue service staff; see Chapter 5 . 


\section{Planning of Fire and Rescue Services}

In this chapter, planning problems that exist within the fire and rescue service, with particular focus on resources management, are reviewed and structured. Studies are structured in order to describe the types of planning problems that previously have been studied and the planning challenges that face the fire and rescue service today. The review has also been conducted to identify which planning problems might be interesting to study in the future.

In general, this chapter is based on a selection of research papers and reports. It should be noted that the intention is not to review all the research that has been performed within the subject, but rather to encompass the entire area. That is, it aims to describe what the planning problems are today and which challenges may crystallize tomorrow.

The examined papers cover many types of problems and aspects of fire and rescue resource planning. However, all the papers are not dealing with a specified problem. Most of the papers review fire and rescue service, but where the studied planning problem is applicable to this subject area, other papers are also discussed.

The research work that has been reviewed mostly applies an Operations Research (OR) methodology to the planning problem that is considered. Thus, usually some sort of mathematical model is used for quantitative analysis. In this chapter, the focus is on planning aspects, but some models will also be discussed and compared.

The planning problems studied in this chapter are reviewed and structured according to decision level and type of planning described in the next section. The subsequent section describes the planning challenges within the fire and rescue service area, and the chapter will then discuss different planning problems according to the decision levels as well as the type of planning. In Section 3.6, all the studied problems are summarized in a table according to their decision level and planning characteristics. Before the chapter ends with some final conclusions and recommendations for further studies, input data requirements for solving such planning problems as discussed are considered.

\subsection{Decision Levels and Planning Character}

The studied planning problems are structured according to their decision level, i.e. whether it is a strategic, tactical or operational planning problem. The characteristics for each decision level are given in Table 2. At a strategic level, the decisions usually concern a large part of the organization, and the planning may have long-term effects. Where to locate fire stations or how to design the entire fire and rescue service organization are typical problems planned at a strategic level. Tactical decisions concern short- or medium-term activities. The tactical level normally involves decisions about how to efficiently use the existing infrastructure and how to organize the operation according to strategic objectives. Operational planning concerns very short-term and day-to-day operations. This, for example, relates to decisions on how to dispatch firefighters and fire vehicles. At the operational level, plans are made for current and next-day activities, usually with respect to daily variations in demand. The characteristics related to each decision level are summarized in Table 2. 
Table 2. Characteristics related to each decision level (Leyva, 2011).

\begin{tabular}{lll}
\multicolumn{1}{c}{ Decision level Characteristics } \\
\hline \hline - & Have long-term effects. \\
Strategic & Normally concern a large part of the organization. \\
& - & Tend to have a major financial impact. \\
& organizations. \\
\hline Tactical & For short-term or medium-term activities. \\
\hline & Usually involve how to effectively and efficiently use the existing \\
& infrastructure. & How to organize operations according to the strategic objectives. \\
\hline Operational & For short-term or day-to-day activities. Real-time decisions. \\
\hline & For responding to very short-term forecasts. \\
\hline & Require deep knowledge of the activities and detailed information of \\
& the situation.
\end{tabular}

The studied planning problems are also structured according to the type of planning, i.e. if the study incorporates static or dynamic planning; see Table 3. The reality is dynamic in nature. However, when planning and making decisions, the planning procedure usually tends to exhibit a static or dynamic character. Typically, for static planning, all the information relevant to the specific planning problem is assumed to be known and that the information does not change during the planning process. This means, if solving a planning problem with a static character, the input data does not change after the solution procedure has begun. In dynamic planning, all the information that is relevant to the specific planning problem must not, but can, be known. In dynamic planning, the information may typically change over time, and time is a very significant component. This means that if solving a planning problem with a dynamic character, all the input data may not be known before the solution process begins, or may be changed after the solution process has begun. A summary of the characteristics for static and dynamic planning is presented in Table 3. 
Table 3. Characteristics of static and dynamic planning (Larsen et al., 2007).

\begin{tabular}{|c|c|}
\hline Type of Planning & Characteristics \\
\hline Static & $\begin{array}{l}\text { - All the information relevant to the planning problem is assumed to } \\
\text { be known before the solution process begins. } \\
\text { - Information (input data) does not change over time. } \\
\text { - The time dimension is usually not important. } \\
\text { - Decisions are usually made for long-term planning. } \\
\text { Decisions do not normally change depending on daily } \\
\text { circumstances and conditions. }\end{array}$ \\
\hline Dynamic & $\begin{array}{l}\text { - All information relevant to the planning problem must not be } \\
\text { known when the solution process begins. } \\
\text { - Information (input data) may change over time. } \\
\text { - The time dimension is always essential. } \\
\text { - Decisions are usually made based on the current situation, i.e. for } \\
\text { short-term or real-time activities. } \\
\text { Decisions may change during the planning period, and the plan } \\
\text { may constantly be updated. }\end{array}$ \\
\hline
\end{tabular}

\subsection{Planning Challenges within Fire and Rescue Services}

Planning of fire and rescue services is a complex endeavor. It involves strategic decisions like how the service should be organized and where fire stations should be built. Tactically, it is necessary to decide how many and which type of firefighters and fire vehicles are needed at each station. Finally, operational planning and control includes deciding which resources to send to a specific accident, or how to relocate resources to ensure adequate preparedness in an area for any and every type of alarm. Preparedness for the fire and rescue service is defined and described in greater detail in Chapter 5.

Traditionally, the planning of fire and rescue service has been regarded as a rather static process. Fire stations have, for example, been built at strategic locations, with the aim of ensuring good coverage of a certain area and of the population in that area, i.e. locations so the fire and rescue service can reach the population within a reasonable period of time. Personnel, vehicles and equipment are allocated to these stations. Each station is usually assigned to a primary response area around the station. Whenever there is an accident in the response area, the firefighters in the primary station will jump into their fire engines and rush towards the accident site. Afterwards, they usually go back to the station.

The process just outlined above is highly simplified, but serves as an illustration of the setting for most of the planning problems studied within the field. However, planning fire and rescue service is not static in reality. For example, the demand for the service may vary over time, and the resources are not always available at the fire stations. These factors should be taken into account in a dynamic view. A typical example of dynamic planning is to dispatch resources to accident sites depending on the current situation in the area, and if necessary, dispatch firefighters from different locations. Another example is to relocate resources to 
empty stations or other appropriate locations in order to always be prepared for taking care of new incoming calls.

There have been a number of surveys published within the subject area, although none deals explicitly with the fire and rescue service. Some of them have a broader context like, e.g. the chapter about the deployment of emergency services by Swersey (1994). Swersey discusses planning problems for emergency medical services (EMS) and police, in addition to fire and rescue service, and covers organizational issues, location problems, staffing problems, scheduling problems and dispatching problems, among other elements of the emergency response operation. Goldberg (2004) focuses on the deployment of EMS and fire service, and how to model and analyze these kinds of problems. Goldberg also considers system operations and performance criteria as well as applications and software systems. Larson (2004) spans a broader area, including hazardous materials, bio-terrorism and private sector response into the discussion. Simpson and Hancock (2009) take somewhat of a historical perspective and describe how the operational research work on emergency responses has shifted focus over time. Apart from fire, ambulance and police services, they also consider disaster services, terrorism, earthquakes and pandemics. Emergency responses within the context of disaster operations management are also considered by Altay and Green (2006), who do not focus on daily events, but draw parallels between disaster response operations and the everyday emergency response, making the survey relevant as well.

Furthermore, there exists a number of surveys covering emergency medical services, e.g. Ingolfsson (2013) and Brotcorne et al. (2003). Brotcorne et al. (2003) classify models used to locate and plan ambulance services into two main categories: deterministic and probabilistic models. Deterministic models ignore stochastic considerations regarding the availability of ambulances and probabilistic models consider the ambulances as servers, in a queuing system, which are not always available to respond to a call. The paper considers both early models dealing with static location problems and more recent models dealing with dynamic location and relocation problems. The fire and rescue services have, as mentioned earlier, traditionally been regarded as static, and even today it may be a valid assumption when performing strategic and tactical planning that the resources will most likely be available, often at the station, if there is a new call. However, with the increased desire to use the resources for preventative work outside the station, dynamic location and relocation models that have been used for EMS could also be applicable to the fire and rescue service.

\subsection{Strategic Planning}

Of the three decision levels, the strategic level is the top level. At this level, decisions are usually taken on a long-term basis and may affect the entire organization. Normally, the top management and other important management functions are involved in the decision process. Examples of strategic decisions are to construct emergency facilities, determine service areas or define resource and equipment standards. Strategic decisions tend to have a major financial impact. For example, when building a new fire station, the cost does not only involve the cost of materials and the work to construct the building; it also includes costs for analyzing and forecasting the expected demand for service in the area, planning the station capacity, determining the expected travel times and environmental costs (Leyva, 2011). To facilitate 
good decisions at the strategic level, it might also be necessary to gather information about government incentives, community requirements, local and environmental regulations, etc. Decisions of a strategic character are not likely to change very often.

Much of the research performed within the fire and rescue service area considers strategic planning, focusing on the location and allocation of fire resources. The location of fire stations is by far the most frequently studied problem in the subject area.

An early study of how to decide the optimal number of fire stations and where to build them was presented by Hogg (1968). By assuming that the resources are always available and present at stations, the duration between receiving an alarm and the arrival of emergency personnel at the incident is directly affected by the location of the fire station. Thus, selecting the optimal number of fire stations and strategically positioning them in relation to each other and to areas with high demand will mitigate the effects due to fire. Hogg developed a method for minimizing the total response time to fire accidents for any given number of fire stations. The problem is basically designed as a p-median problem (Hakimi, 1965). However, with the p-median model, areas with low demand can get a quite long service distance to the nearest station. To handle that, Toregas et al. (1971) consider an upper limit on the response time or the distance to all the demand nodes in their model for locating fire stations. This means, that there must be a fire station located within that specified time or distance for each demand node, i.e. they formulated the problem as a Location Set Covering Problem (LSCP). The developed model minimizes the number of fire stations to be located within the predefined service requirements. Schreuder (1981) also used a location set covering model for locating fire stations. Compared to Toregas et al (1971), Schreuder (1981) included that double coverage, i.e. that an area is covered by more than one fire station, is necessary for certain high risk areas. It may be noted that the reason for dual coverage was to reduce the risk of prolonged response times caused by traffic congestion, i.e. not to cover for the fact that a station might be empty. Dual coverage models can also be used to increase the probability that at least one nearby station will have available resources when an accident occurs.

One disadvantage of the LSCP is when the number of resources available to be located is less than the number needed to cover all demand nodes. To deal with this situation Church and ReVelle (1974) extended the LSCP and introduced the Maximal Covering Location Problem (MCLP), which is to maximize the population covered within a specified service standard, e.g. distance or time, by locating a fixed number of facilities. But since the MCLP cover the maximum population possible, no considerations for those who are not covered within the specified service standard are taken. Basically, this means that it does not matter how long it takes to reach them. To ensure that the population not covered by the predefined service standard is not too far away from a station, the authors also formulated the MCLP with mandatory closeness constraints. This extends the problem to cover as many as possible within a desirable service distance $S$, but at the same time ensure that no one is further away than $T$ to its closest facility, where $T>S$.

Schilling et al. (1980) extended the MCLP with a multi-objective formulation of the covering model that considers both population and property values in the demand areas. They also 
describe how other objectives can be incorporated when locating fire stations. In reality, it is not only the stations that should be considered when planning for an efficient emergency response. At the stations, there are also a number of vehicles of different types, e.g. pumpers, ladders or fire trucks that should be taken into account. Shilling et al. (1980) discuss how the covering model can be adapted if two types of fire engines are available at the stations, and also how the fire engines can be relocated to the stations to improve the coverage.

Covering location models for emergency situations that consider multiple response units have more extensively been discussed by Schilling et al. (1979), Batta and Mannur (1990), Marianov and ReVelle (1992) and ReVelle and Snyder (1995). A limitation of the original LSCP and MCLP is that they do not allow different types of resources, e.g. different types of fire vehicles. Batta and Mannur (1990) have extended both the LSCP and the MCLP to locate response units in an area, where some parts of the area require multiple types of units to be within an acceptable distance or travel time to achieve coverage. However, Batta and Mannur (1990) only considered one type of response unit, and all of them are also treated equally, in contrast to Schilling et al. (1980), which consider different types of response units. Schilling et al. (1979) also developed extensions of the LSCP and the MCLP, in particular the FacilityLocation, Equipment-Emplacement Technique (FLEET) model. By developing the FLEET model, they made it possible to also consider various types of vehicles and crews, e.g. both primary and specialized units that can either be located in tandem or by themselves. Basically, they first locate a fixed number of fire stations, and then allocate a fixed number of various fire resources to the stations, for example primary and specialized units. Each station may then provide either a primary or a specialized unit or both. The goal of FLEET is to maximize the population covered according to the units' specific service standards, for example within a special time or distance, which may vary depending on the type of resource to be located. The MCLP is also extended by Marianov and ReVelle (1992). They developed a model to maximize the population or the number of fire calls covered by at least three fire engines and two ladder engines, which they define as the standard response to a fire alarm (within specified standard response distances). In their model they also consider the probability that a fire engine may be busy when receiving a call. The fire stations had a limited capacity, defined by the maximum number of vehicles that could be sited at each station. ReVelle and Snyder (1995) developed a model to locate fire stations where they also considered ambulances. Ambulances are considered in their model, since it was quite common to locate ambulances and fire vehicles together, however, often without any specific analysis of the ambulance coverage. In their model they located fire stations and ambulance stations, subject to a budget constraint limiting the total number of stations and vehicles that could be located. At a fire station, fire engines, fire trucks and ambulances could be located, while at ambulance stations, only ambulances were allowed. The objective was to maximize the population covered by the emergency resources within specific service standards.

As observed from the studied planning problems, it is important to decide how many fire stations to have in an area, and also to make sure that the firefighters can reach the expected incidents within a reasonable period of time or distance. A connection between the number of stations in an area and the response distance has been developed by Kolesar and Blum (1973). 
They found that the average response distance in an area is inversely proportional to the square root of the number of locations where fire engines are available to respond to incidents. They mean that their developed model can be used for determining the required number of places from where fire units should be available to respond at any time, in a predefined area, while maintaining a desired level of response time. Given specified resource constraints and response time standards, it should be possible to find optimal resource allocations. And also, certain response times can then be determined from the proposed allocation plan. It should additionally be possible to use the developed model by Kolesar and Blum (1973) in tactical context. If the purpose is to coordinate resources in the shorter terms, for example to coordinate fire vehicles instead of locating fire station, the resource planning will then likely consider more tactical decisions where the planning will change more often. Tactical decisions normally consider planning for a couple of weeks or month instead of several years as strategic planning; see Section 3.4.

Different costs, such as for procuring vehicles and equipment or for building new stations are important in strategic planning of fire and rescue service. Coskun and Erol (2010) included such costs in their covering model for locating two types of EMS stations with different capacity levels. This problem is relatively similar to the ones presented by Schilling et al. (1979). When locating the stations, the demand nodes must be covered by a certain station under some service constraints, e.g. within a specific travel time or travel distance. Beyond that, Coskun and Erol (2010) also minimized the total cost of the system. In the fire and rescue service area, there are however different types of stations with different characteristics, e.g. full-time and part-time stations. Chevalier et al. (2012) developed a similar model as Coskun and Erol (2010), but for locating fire stations. In their model they also consider both staff and equipment allocations. More exactly, they have developed a decision-aid tool that would help to plan for a better use of emergency resources. As well as determining the fire stations' locations, the developed tool would support to also allocate resources (staff, equipment, engines etc.) and define the stations' service areas. The resource planning is therefore in this problem also to some degree on a tactical level when it deals with the question of how to utilize and allocate the staff, the engines and the equipment. The objective is to minimize the setup costs while the stations will be located to meet a predefined level of service, e.g. to ensure that a given proportion of expected emergencies can be reached within a given time. The setup cost depends on the number of fire stations as well as on the number of vehicles and crews present at each station. Another way to consider costs has been investigated by Halpern (1979). He developed a method to compare the cost effectiveness of installing detection-alarm systems in dwellings with adding fire stations in order to improve the fire protection in an area. He means that instead of adding one more fire station in an area, it can be more cost effective to install detection-alarm systems in houses. The advantage of installing a detection-alarm system is that a fire might be detected in an earlier state. Additional is the provision of more and better information that automatically could be transmitted via the alarm, e.g. correct address and exact location of the fire in the house. This may reduce the response time, the search time for finding the fire and the property losses from the fire. The relation between fire property losses and response time is discussed more in 
detail by Mattsson and Juås (1997). They have made a cost benefit analysis to measure the benefits and the costs for the society depending on the fire and rescue service response time.

The priority levels of the incoming emergency calls are something that quite often is taken into account when locating emergency medical resources. Silva and Serra (2008) presents a location covering model with priority queuing theory, which suggests locations to a given number of facilities to provide a service standard for the different priorities. A demand node can then be allocated to a center for one priority and to another center for other priorities. The objective is to maximize the population covered in all priorities. In Sweden and also in many other countries, the calls for EMS are prioritized depending on the urgency of the alarm. However, this is not usually the case for fire and rescue service. In Sweden, the alarms for fire and rescue service are mostly treated equally, i.e. with the same priority. However, it would be interesting and in many cases beneficial to also prioritize the incoming calls for fire and rescue service. This is an area worth further study.

An assumption in the earlier covering location models is that the resources always are expected to be available. This assumption is still common and in fact quite valid for fire and rescue service, where the resources normally are not as busy as ambulances. Of course, multiple simultaneous calls happen also in fire and rescue service which makes it interesting to consider probabilistic models and taking into account the fact that a unit might be busy when the next call arrives. These are quite common consideration for EMS and other response services. Geroliminis et al. (2009) for example, present the Spatial Queuing Model (SQM) for locating emergency vehicles in a congested system. The model is developed for locating vehicles in urban networks considering both spatial and temporal characteristics of demand and the probability that a server is not available when required. This paper is further discussed in Section 3.4, since it is mainly applicable when considering decision making and planning at a tactical level. Another probabilistic model for locating emergency stations, is presented by Beraldi and Bruni (2009). They focus on the uncertainty of the demand and assume that the main source of uncertainty comes from the emergency call process. Congestion occurs when the requirements of resources from service calls, arising from an area during a given time interval, exceed the number of available resources. Similarly as in Geroliminis et al. (2009), the average traveling distance is used to determine if a station can provide service to a demand point in time. When locating the stations they also include a fixed cost for opening a station and a vehicle capacity cost per station, similarly to Coskun and Erol (2010). In the model developed by Beraldi and Bruni (2009) emergency response vehicles will also be assigned to the stations in a manner that ensures an adequate coverage and a quick response time to the incidents. Thus, the model presented by Beraldi and Bruni (2009) is also used for tactical planning.

When planning fire and rescue service, the most common objective is to reach the demand, or the population, as quickly as possible. However, there are many more things that can be considered during the planning process. Badri et al. (1998) for example, consider a total of eleven strategic objectives in their model for locating fire stations. Their multi-objective model includes among other objectives, minimizing average and maximum response time, minimizing average and maximum distance traveled, maximizing service to areas where most 
fire accidents occur, minimizing station overlaps and avoiding locations where water availability could be a problem. The model also considers different costs for providing the service. Another multi-objective method to determine the optimal number of fire stations and where to locate them is developed by Yang et al. (2007). Focus of this paper is to consider the various fire risk categories of a given area and to establish a practical fire station location model to fire service authorities. To determine the optimal number of fire stations to locate in a region, they have formulated a couple of objectives such as minimizing the total setup and operating costs of fire stations and the total loss cost of accidents in a given area. The fire stations locations are then determined by minimizing the longest distance from a fire station to any expected accident site by using a fuzzy multi-objective model.

It is also important to make sure that the right type of equipment and expertise arrive at the accident site in time. This is considered by Berman et al. (2005). They present a methodology for determining the optimal locations of specialized response team stations to maximize their ability to respond to incidents in a region within a threshold time. For locating the specialized stations they use a maximal arc-covering location model. It means that each road link requires emergency response coverage and a set of arcs make up the transport network rather than a set of points. Normally, when planning fire and rescue service response, the population density is considered and not the road links. It could however sometimes be interesting for the fire and rescue service to include the road links, since it can e.g. be important for regions with busy roads or for roads where a lot of hazardous substances are transported. Furthermore, incidents at roads may also sometimes need specific equipment or expertise from the fire and rescue service. Lately, the fire and rescue service also responds more frequently to traffic accidents than a few years ago.

\subsection{Tactical Planning}

The time horizon for tactical planning is in general less than a year and tactical decisions are likely to be changed or reviewed periodically. Tactical planning of fire and rescue services may include personnel planning, resource allocation, resource utilization, equipment management or how to manage an incident. Typical costs may be related to new resources, new equipment, fleet size changes, route diversions or other changes in the operations. A tactical question could for example be how to best utilize the available fire resources for obtaining and maintaining control of incidents (Svensson et al., 2005). In some studies, strategic and tactical planning is combined. The combination between the strategic question of where to locate fire stations and the tactical concern of how to equip the stations are presented in e.g. Schilling et al. (1979), Beraldi and Bruni (2009), Coskun and Erol (2010) and Chevalier et al. (2012). These studies are mainly described in Section 3.3.

A simulation model that works as a support tool for resource allocation and management of an emergency service system is presented by Zaki and Cheng (1997). The area under consideration is divided into a number of zones and data such as the expected number of incoming calls, the zone's geographical size, population density and the time of the day are used. The model considers response priorities which are assigned to the incidents depending on its degree of severity and urgency. According to Zaki and Cheng (1997), by using the developed support tool, it should be possible to evaluate different tactical decisions like how 
to allocate available resources and how resources should be dispatched to incidents. When Andersson and Särdquist (2007) present a model for locating fire and rescue service resources, they also consider the fact that it may be beneficial to relocate resources with respect to temporal demand changes like Zaki and Cheng (1997). However, Andersson and Särdquist (2007) do not consider as many factors as Zaki and Cheng (1997). On the other hand, Andersson and Särdquist (2007) consider multiple accident types and multiple resources e.g. different fire vehicles, and they link an accident type to a specific set of required resources. The model makes it possible to analyze and compare different ways of organizing the fire service. Typical scenarios to analyze can be the effect of mixing traditional groups of $4-5$ firefighters with smaller groups of firefighters, or considering locations where the demand of service may vary daytime from nighttime. They have used three different models to find optimized locations for a set of specified resources. The first one is a $p$-median model, the second one is a p-center model and the third one is a maximal covering location model. By using different models, three different types of solutions are obtained and compared. The overall purpose is to facilitate and support the tactical planning process. This purpose is also noticed by Dahlgren et al. (2009). They present a method for planning where non-stationary units are considered, i.e. resources that are not confined to a certain location but may move freely within a specific area. In the paper they evaluate fire and rescue resources capabilities and availability when non-stationary units also are available. The nonstationary units typically consist of smaller units that also were considered by Andersson and Särdquist (2007). In the used method, the non-stationary units could be located at various places in an area, while the stationary units only could be located at the fire stations. Different scenarios of possible locations for the non-stationary units are tested and presented in the paper by Dahlgren et al. (2009). In order to measure the geographic accessibility they evaluate the risk level in an area, the mean response time and the concentration (the number of persons that currently can be at the incident location as a function of time). They found that, by using non-stationary units, a more flexible and efficient use of rescue units may be obtained, and also a decreased response time. Another model to tactically coordinate emergency resources is presented by Schmid and Doerner (2010). To ensure continuous good coverage they consider time-dependent travel times. The travel speed can thus be different for different street segments, during peak hours and it can vary considerably during the day. The objective is to provide the decision maker with time-dependent location plans, such that the resulting coverage can be kept at a required level during the planning horizon. The problem they describe is to locate and relocate ambulances, but it should be directly applicable for fire and rescue service as well.

As discussed in the previous section, it may also sometimes happen that resources are not available when requested. This is more commonly considered when locating ambulances, e.g. like in Geroliminis et al. (2009), due to the frequent alarm rate in EMS. The model developed by Geroliminis et al. (2009) aims to tactically locate vehicles in an urban network considering both spatial and temporal demand characteristics such as the probability that a server is not available when required. The objective is to minimize the mean response time while the coverage in the area has to be greater than a predefined value. Beraldi and Bruni (2009), whose work is further described in Section 3.3, also consider uncertainty in the availability of 
the resources, foremost when strategically locating emergency stations. However, they also consider the tactical assignment of allocating emergency vehicles to the stations.

A common way to facilitate dispatch decisions, i.e. to decide which resources to send to an incident, is to define response areas for each station or resource. A response area represents the area of responsibility for a specific fire station or a specific resource in case of an emergency. Response areas might differ in size and population density but also depending on resources availability. For example, if the resources at one station are busy, another station might have been assigned a larger response area. A model that considers the tactical challenge of finding response areas for fire stations is developed by Yang et al. (2004). Their goal is to find primary response areas for fire stations with two different capacities of available fire companies (stations with two or four companies). The objective is to minimize the average response time to incidents and also to balance the workload of the fire stations. Carter et al. (1972) also developed a model for finding response areas, in their case for units instead of stations, although the results are easily transferable to stations. They show that if the alarm frequencies between two response areas differ, it may be beneficial to expand the area with the lower frequency to balance the workload and decrease the risk that the busier unit is unavailable when a new call arrives. Thus, for some calls, the closest unit will not respond, and this will minimize the response time in the long run.

Another example of tactical planning is how to perform the actual firefighting at the scene. Firefighting consists of many different activities and strategies that usually depend on the characteristics of the incidents. Halpern et al. (1982) present a method for the analysis of manning strategies in firefighting operations. They break the operations down into distinct and well-defined activities so that the fighting of any fire can be described in terms of those activities and the precedence relationships between them. The sequence of activities may also be affected by the number of firefighters needed on the scene. When resources are limited, it may for example be necessary to delay the execution of an activity due to a lack of manpower. Selection of activities should be made in order to minimize the total execution time. The objective is to find a relationship between the number of firefighters on the scene and the time required to extinguish a fire, and the model can be used to evaluate the effectiveness of different manpower strategies. A different way to evaluate the effectiveness of firefighting is made by Bishop et al. (1971). They have developed a model to compare the response capabilities in form of manpower, the firefighters' quality, the vehicles' performance, available equipment and the response time with the demand for fire and rescue service within a service area. The developed model is constructed to estimate the probable use of fire services and then measures the capabilities of providing it. The purpose is to facilitate the resource planning by evaluating the effectiveness of firefighting. The evaluating is made in order to utilize fire resources in a given area in the most effective manner. The authors mean that when a certain level of effectiveness is determined, the evaluation can be used to efficiently allocate the available resources in relation to expected incidents. Both Halpern et al. (1982) and Bishop et al. (1971) have developed methods for determining the effectiveness of firefighting, but they use different definitions. Halpern et al. (1982) deals with the actions 
on the accidents site while Bishop et al. (1971) compare the requirements for an effective protection with the present available response ability.

In order to adequately respond to emergencies and maintain a good geographical coverage, manpower planning is an important tactical component for fire and rescue service. It is essential to always have enough resources to manage expected fires and other emergencies. Manpower planning is a rather complex operation since shifts for example can vary in length and starting times. Church et al. (2001) discuss the manpower planning problem for emergency services. They focus on how to determine the best combination of staffing and equipment over time and space in order to effectively meet the demand. To solve the manpower problem, they divide it in two main components. First is identifying the appropriate level of staffing by crew-shifts over hours of the week and then locating and allocating appropriate type of equipment to meet service demands over time. The problem is somehow associated with identifying just what is needed in terms of equipment and personnel in order to meet some desired set of service standards, e.g. in order to be able to respond to accidents within a certain period of time or distance. The personnel scheduling problem can become rather complex, however, when shifts vary according to number of days, duration of working hours, and starting times. The number of potential solutions also grows with the concomitant increase in the number of shift types and starting times. Church et al. (2001) present two examples of models for solving the personnel scheduling problem.

\subsection{Operational Planning}

Normally, operational decisions have a time-range of days, hours or minutes. It is decisions made for the daily operations. New technologies have made it easier to make decisions in real time, provided that the required information can be obtained by various online systems. The decisions usually influence the organizational areas currently involved in the actual operational work.

Costs associated with decisions at the operational level are mainly consumables and usage costs like extra fuel costs, costs for using additional resources, overtime costs, costs for handling additional equipment or costs for handling online information. The costs for planning and organizing operational decisions are usually not as significant as for strategic and tactical decisions. Operational decisions normally require much more detailed information than strategic and tactical planning. The relevant information must be updated regularly, since it may change frequently. Also, a rather small part of the organization is involved in the decision-making process compared to the other two decision levels.

For fire and rescue service, operational planning consists of, for example, decisions about how to dispatch, allocate or relocate fire and rescue resources depending on the current situation, the demand of service and the ongoing rescue operations. Operational planning includes the planning of resources, i.e. how they should be utilized and organized in different areas and how they should be used at the accident site. At the operational level, the decisions are likely to change over time because they are usually only effective for a certain period of time. In order to facilitate decision making at this level, the dispatcher should access real-time information regarding available and occupied resources, the situation at the accident site, or if 
an area lacks resources. Such information makes it easier to decide which resources to dispatch to an incident or to find the most appropriate resources to relocate if necessary. It is important to both conduct and coordinate efficient responses to accidents as well as to be prepared for new incoming alarms all the time.

To decide which resources to send to an incident, models for determining response areas have, for example, been developed by Carter et al. (1972) and Yang et al. (2004); see Section 3.4. These developed models are, however, aimed at making tactically decisions. Carter et al. (1972) and Yang et al. (2004) consider developing response areas for midterm planning. But since response areas sometimes vary in size depending on alarm frequency, it should be possible to also develop and use response areas for short-term planning, e.g. for dispatching resources. Such response areas will then be updated and changed depending on the current conditions and available resources. In that way, it should be possible to use the models developed by Carter et al. (1972) and Yang et al. (2004) for operational planning of fire and rescue resources.

One of the first papers considering emergency planning at the operational level was written by Kolesar and Walker (1974). They developed an algorithm for the dynamic relocation of fire companies. If fire companies have left the fire stations to fight one or several fires, it may result in a sharp degradation in fire protection in one or several areas. If that is the case, it might be beneficial to relocate some available resources to the unprotected area. By storing up-to-date information about the status of all fires and other accidents in progress as well as the locations and the activities of all fire resources, the algorithm developed by Kolesar and Walker (1974) can evaluate, generate and compare many alternative relocation suggestions. The model gives suggestions about when relocations should be made, which empty fire stations should be filled and which available units should be relocated. In the study, they describe that the relocation procedure depends on the expected duration of the current incidents, the expected response time to new incoming alarms, the risk situation, the coverage criterion, the expected duration of the relocation, the need for relocation and the travel distance to a possible relocation site.

Another important operational problem is to decide which and how many fire vehicles to send to an incoming fire alarm. Swersey (1982) has developed a decision rule for how many vehicles to send to a certain incident. The decision rule considers the urgency of the incident, the expected alarm rate in the surrounding area and the number of available vehicles in the area. The objective is to minimize the response time to the incidents. The model only answers the question of how many vehicles to send to an incident and does not answer specifically which one or what type of vehicle/vehicles. An extension to this model is presented by Ignall et al. (1982). They describe an initial dispatching algorithm based on Swersey's insights but consider both how many and which vehicle to send to a fire incident. The objective is likewise to minimize the response time to incidents. The decisions are based on the seriousness of the incident, the alarm history, the time of the day and season, the expected loss of an incident, expected loss at other alarms in the near future and the workload cost. Andersson and Värbrand (2007) have also developed a model that gives suggestions about which unit to dispatch to an accident, and also a model to give suggestions about when relocations should 
be made, similarly to Kolesar and Walker (1974). The models developed by Andersson and Värbrand (2007) are developed for managing ambulances, but since the operations are similar to fire and rescue service, the concept may also be applied there. Which ambulance to dispatch in Andersson and Värbrands' model depends on the priority of the emergency call, the travel time and the change in the preparedness i.e. how prepared the current resources are to handle a certain type of incident. If the preparedness level drops below a certain threshold value, the relocation algorithm tries to find a new location for one or more resources in order to improve the preparedness. The purpose of the relocations is to all the time strive to maintain a good preparedness in an area. The developed decision support tools will give the dispatcher recommendations and suggestions of resource assignments and relocations in real time. Relocations and dispatching decisions for emergency vehicles are also considered by Haghani and Yang (2007). They present an integrated emergency response fleet deployment system which assists the dispatcher in assigning emergency vehicles to emergency calls. Compared to Andersson and Värbrand (2007), the dispatching strategies allow dispatched emergency vehicles to switch route and be dispatched to a new emergency call that is more urgent as well as to change route to a destination based on the real traffic information. The relocation strategy is to relocate the idle vehicles in order to maintain a proper coverage for the expected demands and to achieve shorter response times. Assigning and relocation are considered simultaneously as considering the priority of emergency calls.

Operational planning also considers decisions regarding the rescue effort on the accident site. Dimopoulou and Giannikos (2004) have for example developed a framework for forest fire control and $\mathrm{Ng}$ and Raff (2007) have been discussing the firefighter problem. The system developed by Dimopoulou and Giannikos (2004) consists of a combination of simulation based technique with optimization models and a geographical information system (GIS) module which can be utilized as a flexible tool to assist planners in the design of a complete strategy for forest fire management. The mathematical programming module is used to determine the number of needed resources, where they must be placed, or how they should be rearranged in order to control the fire effectively and to achieve the maximum possible coverage. The simulation module is used to experiment with alternative scenarios and to decide how the firefighters should be relocated according to the behavior and the development of a particular fire. The GIS application stores, updates, retrieves and displays geographic information related to the forest area in question. The idea of the firefighting problem presented by $\mathrm{Ng}$ and Raff (2007) is to determine if it is possible to position firefighters in a way that prevents the fire from spreading any further. The goal is to position the firefighters so that the fire can be controlled after $t$ time periods, where $t$ is minimized.

\subsection{Static Planning}

Static scenarios are almost always a simplification of the real situation, but since the real world is dynamic, constant changes are unavoidable. However, in many cases where the dynamic aspects are not dominant, this is an acceptable simplification, and static planning can yield very useful results. Real world problems are often solved in a static environment and most of the reviewed papers in this survey consider decision making for static planning. 
Something that has a static character does not change in the near future. In static planning it is assumed that all the relevant information needed is known before the planning process begins. That means, in a static approach, all the parameters are assumed to be constant, e.g. travel times, demands, service times and vehicles' availability. All the input data is thus considered to be known in advance and will not change during the planning process.

Relevant information for solving a fire station location problem may for example include a number of possible candidate locations where stations can be placed, the number and types of available fire units at each station, the travel time or the distance between all relevant places and the expected demand in the area. Information such as how the resources can be utilized and moved and other possible resource restrictions must also be known. In static planning, this kind of input information will be considered constant, i.e. it will not change during the planning process. The planning process is also normally bounded, i.e. it starts and ends at some point/time. When solving a planning problem with static character, it may likely result in a high quality solution in a few hours and in some cases also an optimal one. Since problems with a static character also have static objectives, such as minimizing the total distance traveled or maximizing the population covered, it is also easy to compare solutions with different characteristics, for example, solutions with different number of available resources.

All the reviewed papers regarding planning at a strategic level are also aimed for static planning. The papers mostly consider different kinds of location problems where the purpose is to determine where to locate different numbers and types of emergency resources in order to utilize the resources more effectively. The solutions from these problems are applicable for long term planning, where the conditions do not change very often. When for example plans are made for locating emergency facilities, the facilities' locations would not be changed in the near future. The input information for all studied location problems that consider strategic planning is known before the solution process begins and the information does not change or has to be updated after the solution process has begun. When the problem is solved, the resources' location will not be changed. However, some problem characteristics can be changed and different solution can be produced and compared for the same fundamental problem e.g. the number of stations to be located can be varied. The problems presented by Kolesar and Blum (1973) and Halpern (1979) that considers different connections and relationship to find the number of fire stations needed in an area, depending on different conditions, are also structured for static planning when things do not change very often.

The papers that are categorized regarding decision level and type of planning are presented in Figure 6. It should be noticed that some of the papers consider multiple levels and planning characteristics, as discussed below. In this case it will be represented in all relevant groups.

The resource allocation problems presented by Bishop et al. (1971) and Zaki and Cheng (1997), the manning level problem presented by Halpern et al. (1982), the manpower planning problem presented by Church et al. (2001), the districting problems presented by Carter et al. (1972) and Yang et al. (2004), the location problems presented by Andersson and Särdqvist (2007) and Geroliminis et al. (2009), the rescue service planning problem presented by 
Dahlgren et al. (2009) and the ambulance location problem presented by Schmid and Doerner (2010) consider decision making at the tactical level. When solving these problems, no conditions or any information required for solving them will be changed during the solution process. The input information is also known in advance, before the solution process begins. The main purpose of the results of these problems are for a mid-long term decision making and for static planning, i.e. when a result is found it will not be changed in the near future. Thus, all the studied problems that consider tactical planning are also useful for static planning. However, some of these papers - as indicated in Figure 6 - can probably also be used for dynamic planning, which will be further discussed in Section 3.7. Furthermore, the tactical districting problems presented by Carter et al. (1972) and Yang et al. (2004) could certainly also be used for operational planning as discussed in Section 3.5.

The combination of static planning at an operational level means basically it is already predetermined how short-term decisions should be made given a certain situation. This issue does not seem to be well-studied in the literature. Thus, no papers that directly consider operational decision making for static planning have been found.

However, examples that consider static planning at an operational level have been discovered during the interviews made with Swedish fire and rescue service; see Chapter 2. It is for example possible to have different predefined rules regarding which fire station to alert depending on the specific incident, or which resources, e.g. vehicles, to send to a specific incident depending on the situation and where the accident occurred. Different stations might for example be alerted for different situations, different types of accidents and/or depending on when and where the accident occurred. Also how resources should be dispatched to incidents in various situations can be predefined. For example, there can be different predefined guidelines e.g. alarm plans; see Section 2.5, of how resources should be dispatched depending on the type of competency that is needed or how many resources that is needed for a certain type of accident but also depending on which resources that currently is available in the certain response area. Predefined rules can also be made for which emergency resources to relocate in different situations and depending on different circumstances. For example, if one station's resources are busy and the preparedness in that specific area is inadequate, it is quite common that it is predetermined which and how other stations' available resources should be relocated (usually to a fire station) to improve the preparedness (i.e. if they should be relocated). It may also be predetermined if part-time firefighters should be called in, due to a certain situation, for being standby at any particular fire station. Fire and rescue services might use such different predefined rules and guidelines in their operational planning. 


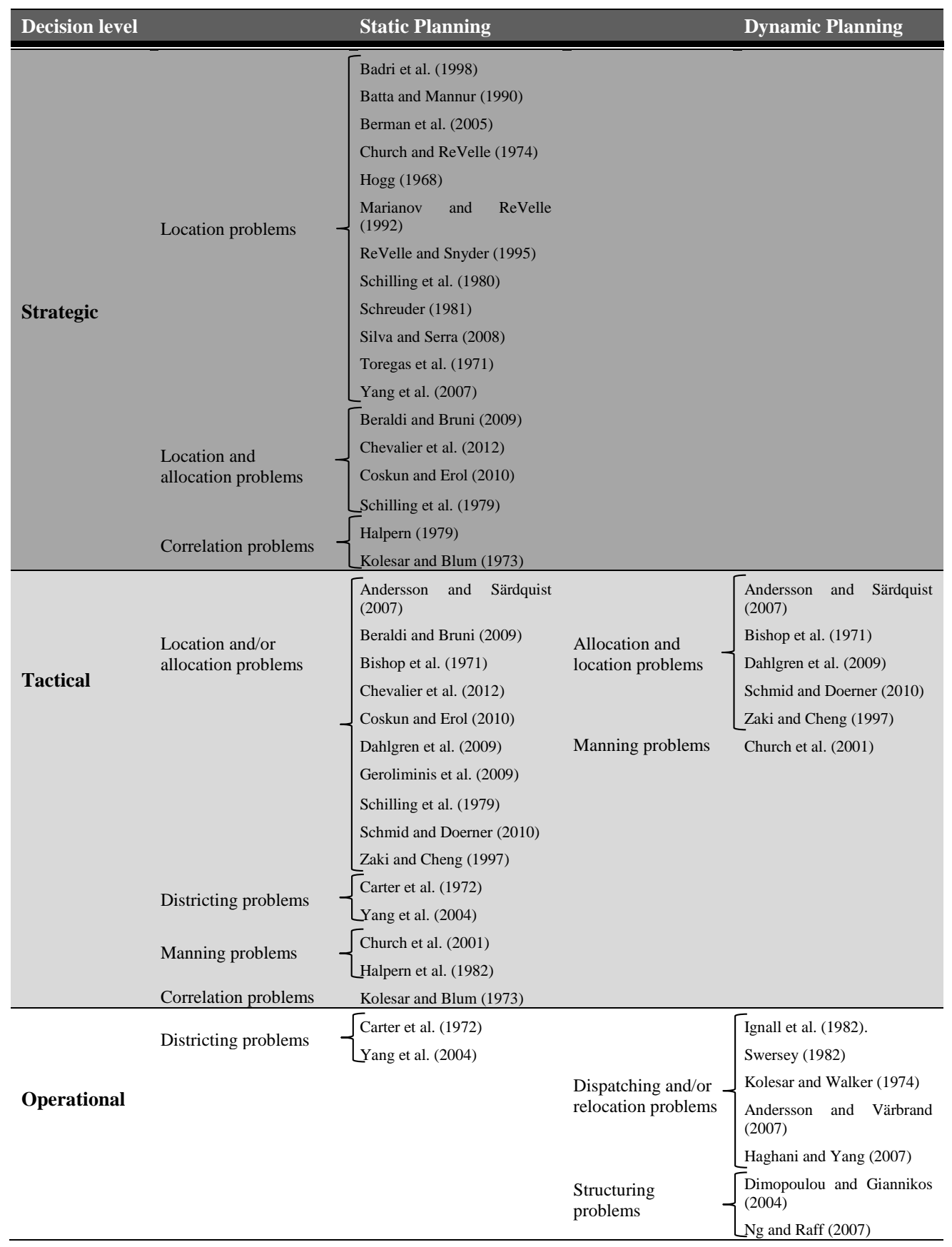

Figure 6. How the surveyed studies are associated to the decision levels and the types of planning.

\subsection{Dynamic Planning}

In dynamic planning the plan will be updated as conditions for the planning have changed. Input data is revealed over time and parameters such as travel times, demand, available 
vehicles, traffic conditions and available equipment might change during the planning period. An example of dynamic decision making can be to decide which resources to dispatch to an accident or decide how to relocate resources. Another example is if a dispatcher has sent a specific fire vehicle to an incident, and in the meantime a new alarm is received, the fire vehicle just sent to the first accident can then be redirected to the new incoming alarm. Another vehicle will then be sent to the first incident.

When solving a problem in a dynamic setting, immediate requests may appear in real-time after the solution process has begun and the information will generally be updated continuously. The input information is normally just valid for a short time. Therefore, it is important that the information update mechanisms are integrated into the solution method. The user usually wishes to know the solution to the current problem as soon as possible, preferably within seconds or minutes. Real time problems as dispatching, rerouting and reassignment problems are for that reason often solved by using different heuristic methods, to get a solution quickly. A problem has dynamic nature if one or more of its parameters are a function of time, i.e. it is a problem with time-dependent data. It means that a corresponding model explicitly includes interaction of activities over time and that the model is usually solved repeatedly as new information is received.

Some of the studied papers in this survey consider dynamic planning. They are not as numerous as those involving static planning, but are increasingly common. All the papers concerning planning at an operational level include also examples of dynamic planning. These operational problems consider short term decision making where information constantly is changing, and the current situation is a base for the planning process.

The operational problem presented by Swersey (1982) describes a dynamic decision rule for deciding how many fire resources to dispatch depending on a number of factors. A similar problem is presented by Ignall et al. (1982). They also describe an algorithm for dispatching fire resources. The algorithm gives suggestions about how many resources to send to an accident but also which fire resources to send to the accident. The algorithm is designed to be implemented in a real-time dispatching system which is based on the information available when the decision must be taken. For both of these problems the time dimension is very important and the information need to be updated frequently since the situation is constantly changing and the dispatcher must make quick decisions when requested.

Another problem that consider dynamic planning, is presented by Kolesar and Walker (1974). They describe an operational method for dynamic relocation of fire companies. Here it is very important to store up-to-date information about the status of all the incidents in progress and the locations of all the fire companies in order to be able to generate and compare different alternative relocation plans quickly. The same applies to the dispatching and relocation problem presented by Andersson and Värbrand (2007). This is also a problem with dynamic settings and up-to-date information about the location of resources. The urgency of the calls and the preparedness in the area is also needed in their algorithm for planning ambulance dispatch and relocation. Furthermore, the problem about assigning emergency vehicles to incoming alarms presented by Haghani and Yang (2007) consider dynamic planning. They 
also discuss the access to real-time information, which makes it possible to avoid congestions, reroute vehicles to avoid delays and reroute dispatched emergency vehicles on route to an incident to a new emergency call that is more urgent.

Real time information is also needed in the system for forest fire control described by Dimopoulou and Giannikos (2004). This study considers decision making at an operational level where online information is important to plan the firefighting and the firefighters' reallocations effectively. The system they describe gets dynamic input data about how the fire spreads and simulates the behavior of the fire depending on speed and direction of the wind (which might change with the time). It is then possible for the system to give suggestions on how and where the firefighters should be placed and sometimes rearranged in order to control the fire effectively. The firefighting problem presented by $\mathrm{Ng}$ and Raff (2007) is also about fire control and to determine if it is possible to position a number of firefighters in different places to prevent the fire from spreading. In this problem, it is very important to always have information about the number of firefighters available, the condition of the fire and how it is expected to spread.

There are no papers considering decision making for planning at a strategic or a tactical level that are intended for dynamic planning. Since strategic planning normally consider decision making for a long period of time (usually several years), information and input data relevant for the planning is usually known or forecasted for the entire time period long before the planning process begins. Conditions and input information does not frequently need to be updated since the information does not change in the short term, time is thus not normally a significant component in strategic decisions. Strategic decisions are basically not made when planning dynamically. As mentioned in Section 3.6 it is probably possible for some of the tactical problems studied in this chapter to also be basis for dynamic planning where the input information is continuously changing. In this case, it should be possible to resolve the models with the new obtained information in order to make new plans according to the new situation. For example, it is possible to find new locations for vehicles and relocate them according to new situations and conditions, and also to make new dynamic schedules for firefighters. Compared to fire stations, vehicles and firefighters can be moved quite easily, and staffing schedules can also be changed in the short term. Solutions to some of the tactical problems could then probably be used for shorter term decision making where conditions and input information might change in the near future. Therefore, the resource allocation and/or location models presented by Bishop et al. (1971), Zaki et al. (1997), Andersson and Särdqvist (2007) and Schmid and Doerner (2010) as well as the manpower planning model presented by Church et al. (2001) and the resource planning tool presented by Dahlgren et al. (2009) could also be used for dynamic planning. Situations when these tactical problems can be used for shorter term planning and more dynamic decision making is for example when extra resources are needed for some reasons, e.g. when there are special events to consider, or to prepare for extreme weather conditions. In such situations, factors like for example travel times, demands, available resources and traffic conditions might change compared to the normal state. This means that new decisions need to be taken and new plans have to be made in order to be prepared for the new situation. 


\subsection{Input Data Requirements}

When making plans for emergency services, different types of input data and information are necessary. The information may depend on the type and the characters of the plan to be made, or the problem to be solved.

Typically, the input data required for almost all the studied problems is some kind of demand in order to know where in the area fire protection is needed. To facilitate the solution process, the area is usually divided into a number of zones or nodes. The demand for fire services then implies the need in the particular zone or node. The demand can for example consist of the number and types of resources that is needed for an expected accident (Batta and Mannur, 1990; Marianov and ReVelle, 1992; ReVelle and Snyder, 1995). The nodes can also include the population, the frequency of calls, risks or the number of expected incidents. The type of information required in the demand nodes depends on the specific problem and the importance of information for solving the problem. In covering problems, the purpose is basically to cover as much demand as possible within a certain time or distance (Schilling et al, 1980; Church and ReVelle, 1974). When solving other problems, as for example manpower planning it might be important to also consider different schedules, shifts and combinations of teams (Church et al., 2001). Other factors that may be needed as input for problems when planning for an efficient fire protection are the capacity of the resources, age of the vehicles, the vehicles' condition, the firefighters' experience and other performance conditions (Bishop et al., 1971). Also, the different types of activities needed to be performed at the accident site are sometimes requested (Halpern et al., 1982).

Similarly to the demand nodes, a set of nodes denoting where fire stations and fire vehicles can be located needs to be defined. These nodes are often the same as the demand nodes, although when locating stations, it may be beneficial to use a smaller subset of nodes as potential location candidates, since there often exist a number of restrictions for where stations can be located.

Since it is important to quickly reach accident sites, the travel time and the distance are significant factors when planning fire and rescue resources. This information is for example used to analyze which of the nodes that can be covered within a certain time or distance, or to minimize the mean travel time to every node. When solving optimization problems, the travel information is usually pre-calculated, i.e. a matrix of travel times or the distances between all nodes is constructed. Since the demand and the potential facility nodes certainly are known, the travel time between them can be found for example by shortest-path calculations on a suitable road network, or by obtaining them from any organization providing road databases. Other options for obtaining the travel times, adapted for fire engines, has been explored by Kolesar et al. (1974). They investigate the relationship between fire engines travel times and the travel distance and then develop an expression for travel time as a function of distance. The expression is derived by using basic mathematical relations and assuming constant acceleration and deceleration during the initial and final phases of travel and a constant cruising velocity during the middle phase. 
Input data might also consist of the number and types of resources, e.g. fire stations, vehicles, firefighters or various equipment available to locate, reallocate or dispatch (Silva and Serra, 2008; Schilling et al., 1979; Yang et al., 2007). For some problems it is essential to also know where the resources currently are located, e.g. for relocations and dispatching problems (Kolesar and Walker 1974; Ignall et al., 1982). Sometimes, the problem is instead to determine the number of resources to locate and decide where to locate them (Toregas et al., 1971; Batta and Mannur, 1990) or decide where to locate a predefined number of resources (Church and ReVelle, 1974; Schilling et al. 1980). For the latter types of problems, various service standards are often used, e.g. requirements on response time, travel distance or coverage of the population. An example could be that the expected response time within an area never should exceed 30 minutes or that the travel distance never should exceed 10 kilometers.

When planning for an efficient fire protection, costs can sometimes also be included, for example set up and operating costs of fire stations. This is then also an input parameter when solving fire resource location problems in order to minimize costs (Badri et al., 1998; Yang et al., 2007; Coskun and Erol, 2010). When planning in a short term perspective, as for tactical and operational problems, it is also important that the input information constantly is up to date. Other information that can be necessary for solving various planning problems, especially in the short term, can be the time of the day, traffic congestion, weather conditions, expected operation time, the accidents' behavior, the firefighters' and the fire vehicles' location etc. (Dimopoulou and Giannikos, 2004; Zaki and Cheng, 1997; Schmid and Doerner, 2010).

\subsection{Conclusion}

Planning of fire and rescue service can be strategic, tactical or operational. It is also possible to make a distinction between static and dynamic planning. In the work reviewed here, decision making at a strategic or tactical level is normally intended for static planning, just as can be expected. For example, the most commonly studied problem of locating fire stations is a typical strategic problem, where the input data needed to solve the problem is not expected to change in the short term. Similarly, operational decision making - including dispatching and relocation decisions - is mostly done for dynamic planning, where continuous changes in the setting and the input data are to be expected. However, some of the tactical problems that is primarily intended for static planning can also be used for dynamic planning. One example is manpower planning, where a static base plan can be constructed, but the same model can be used for adapting this plan when required by a change in demand. Likewise, it is also possible to find operational problems that consider decision making for static planning, for example predetermined dispatch rules, although in the work reviewed here, these are not common.

From the selection of work reviewed, it is evident that most of the research support a strategic and static fire and rescue service planning. The reason might be that the fire and rescue service traditionally has been characterized by relatively static planning and resource management. Emergency resources have been strategically located in populated areas and the 
firefighters have been waiting at the station until they have received an alarm, and when they have completed a mission, they return back to the station and wait for another call. In these situations, the most evident planning challenges are to determine how many fire stations there should be in an area, where the stations should be located, their capacity and amount of resources required at the stations, and other strategic decisions.

However, the conducted interviews described in Chapter 2, as well as the increasing amount of research concerning dynamic planning, indicate that a new way of approaching the management of fire and rescue resources is gaining ground. As mentioned in Chapter 2, many of the interviewed fire and rescue service state that the fire and rescue services in Sweden have started to deploy smaller units instead of only relying on the traditional fire units with five firefighters. The reason is to be able to use these units for preventative work as well as maintaining adequate first response coverage in the area. Using smaller units that cannot be expected to be found at fire stations when there is an alarm, gives rise to an increasing complexity in the planning process. It becomes important to keep track of both the vehicles, and the individual competencies of the firefighters who are manning the vehicles. If, for example, a certified operator of a ladder truck has been assigned to a small unit to make an inspection, it may be necessary to route him or her first to the fire station to pick up the ladder truck, before proceeding to an accident. These type of decisions can be somewhat supported by the research performed in the reviewed work, concerning tactical and operational planning, but more work is needed to facilitate such decisions.

Planning of fire and rescue service today is moving toward a similar situation as planning emergency medical service (EMS). In EMS planning it has long been assumed that it is never certain that an ambulance will be available where there is a new a call. Much of the EMS research also assumed that the ambulance may be available outside of the ambulance station, on its way to a patient, or back from the hospital. The same is starting to become true for fire and rescue service, but with the added complexity that the personnel cannot be assumed to all have the same competencies, and the vehicles have different capabilities. Furthermore, different combinations of personnel competencies and vehicle types are required for the wide range of events that the fire and rescue service are expected to handle.

In conclusion, more research is needed concerning dynamic planning of fire and rescue service; operationally to support decisions about for example which resources to dispatch and how available resources should be relocated to maintain an adequate level of preparedness, but also strategically and tactically to adapt the fire and rescue service operation when it is no longer possible to assume that all resources will wait at the fire station for an alarm. Other exiting research opportunities exist also in the planning the use of fire and rescue service resources for preventative work, which could be done more with respect to the preparedness level in the area. Also how other non-traditional response resources can be utilized in fire and rescue service responses is an interesting research issue like e.g. how home care nurses or security guards can be used as first responders to support the fire and rescue service (Weinholt \& Andersson Granberg, 2013). 


\section{Strategic Location of Fire and Rescue Resources}

The time that elapses between receiving an alarm and the arrival at an accident site is directly affected by the location of fire stations (Hogg, 1968). By optimizing the location of fire and rescue resources, it is possible to shorten the time it takes to reach an accident site, i.e. the response time, when an incident has occurred. This can reduce loss of lives, injuries and property damage. Thus, it is essential that resources are available where they are needed.

In previous studies, the problem of where to physically locate fire stations, fire and rescue vehicles and firefighters has often been studied. The location problem typically aims to ensure a short response time, an adequately covered population or a reduction of the total losses from an accident as well as the cost of providing the service (Yang et al., 2007).

In earlier models; see Chapter 3, it is usually assumed that all the stations possess the same equipment and personnel. The individual firefighters' capability or the number of firefighters needed at an accident site is normally not considered. If a station can be found within a certain distance or time, the need for coverage has often been regarded as satisfied. However, in reality there are different types of stations with different numbers and types of firefighters and vehicles. Depending on the type of accident, the number and types of resources requested to the accident may also vary. To achieve an efficient location of resources needed to take care of certain accidents, it is thus essential to include all the resources that are needed for the accidents.

In order to evaluate locations of a given number of fire and rescue resources, an optimization model that gives suggestions on where the resources should be located is developed in this chapter. The model explicitly takes into account the connection between different types of accidents and the resources needed to handle them, i.e. the model can be used for different types of demand. The model locates a number of given fire and rescue resources in order to minimize the response time to expected accidents in a given area. The response times for both the resource that first arrives at the accident site as well as the response time for the last arriving required resource are considered in the model. The response times can also be weighted differently in order to obtain solutions with different characteristics with respect to the response times.

The location model has been solved for a test area where two types of accidents and three types of resources are considered. It is shown how the model can be used for locating resources according to different requirements. Solutions to the model are produced by a reduced variable neighborhood search heuristic (Hansen and Mladenović, 2001). Focus is on producing and analyzing results for scenarios with different characteristics rather than evaluation of the solution method. Results for seven scenarios are presented and the results may serve as a basis for strategic decisions concerning resource management. 


\subsection{A Location Model}

An optimization model has been developed to suggest locations for a given number of different types of response units. A Unit, more described in Section 2.4, consists of both a fire vehicle and one or more firefighters, e.g. one fire vehicle and four firefighters. The units are located with respect to expected accidents in the specific area. In the model it is possible to consider multiple types of response units, i.e. different combinations of vehicles and firefighters, but also multiple types of accidents.

There are no limitations on the number of accidents or units that can be included in the model, but it must be known in advance. For each modeled accident, certain values must be specified, for example in an alarm plan, how many units and firefighters that are required to handle the accident. The model, thus, can be used for various types of fire and rescue service organizations.

For the fire and rescue service, it is important that resources can quickly respond to accidents. The objective function of the model is, thus, to minimize the response time to the expected accidents in a given area. (The response time for a unit is the preparation time plus the travel time for the unit.) However, it is also essential to make sure that all the required resources arrive at the accident site. Therefore, both the first response time and the full response time are considered in the objective function of the model. The first response time is the time when the first resource has reached the accident site and the full response time is the time when all the requested resources have reached the accident site. The full response time, thus, can be equal to the first response time. That happens when all the requested resources arrive at the accident site at the same time.

Furthermore, it is not obvious that both of the response times have the same importance in all contexts, or that one of the times is more important than the other. The time for first responses and the time for full responses, thus, can be weighted differently in the objective function depending on how the user wants the times to affect the result. Weight parameters for the response times are therefore included in the objective function. The weight parameters can be varied to obtain solutions with different characteristics. For example, if it is considered more important that some resources quickly can respond to an accident, than that all the required resources quickly can reach the accident site, the time for first responses can be weighted higher than the time for full responses and vice versa. Via input parameters, it is stated which units that are permitted to perform first responses.

The resources that are available for the model are expected to always be available, i.e. that resources might be busy is not considered.

If the given area is divided into a number of zones, the model is formulated as follows:

Sets:

$O:$ the set of accident types

$I, J$ : the set of zones

$U$ : the set of units

$K$ : the set of unit types 


\section{Variabels:}

$x_{u i}=\left\{\begin{array}{l}1 \text { if unit } u \text { is located in zone } i \\ 0 \text { otherwise }\end{array}\right.$

$y_{u o j}=\left\{\begin{array}{l}1 \text { if unit } u \text { responds to accident of type } o \text { in zone } j \\ 0 \text { otherwise }\end{array}\right.$

$q_{u o j}=\left\{\begin{array}{l}1 \text { if unit } u \text { makes a first response to accident of type } o \text { in zone } j \\ 0 \text { otherwise }\end{array}\right.$

$m_{\text {uioj }}=\left\{\begin{array}{l}1 \text { if unit } u \text { located in zone } i \text { responds to accident of type } o \text { in zone } j \\ 0 \text { otherwise }\end{array}\right.$

$r_{u i o j}=\left\{\begin{array}{l}1 \text { if unit } u \text { located in zone } i \text { makes a first response to accident of type } o \text { in zone } j \\ 0 \text { otherwise }\end{array}\right.$

$F_{o j}=$ response time for a first response to accident of type $o$ in zone $j$

$T_{o j}=$ response time for a full response to accident of type $o$ in zone $j$

\section{Parameters:}

$s_{u k}=\left\{\begin{array}{l}1 \text { if unit } u \text { is of type } k \\ 0 \text { otherwise }\end{array}\right.$

$z_{u}=\left\{\begin{array}{l}1 \text { if unit } u \text { can make a first response } \\ 0 \text { otherwise }\end{array}\right.$

$d_{o j}=$ expected number of accidents of type $o$ in zone $j$

$p_{u}=$ number of firefighters included unit $u$

$t_{i j}=$ travel time between zone $i$ and zone $j$

$a_{u}=$ preparation time for unit $u$

$B_{o}=$ required number of firefighters to accident of type $o$

$E_{k o}=$ required number of units of type $k$ to accident of type $o$

$\alpha=$ the weight parameter for first response

$\beta=$ the weight parameter for full response

Min $\quad \sum_{o \in O} \sum_{j \in J}\left(\alpha F_{o j}+\beta T_{o j}\right) d_{o j}$

S.t.

$$
\begin{array}{ll}
\sum_{i \in I} x_{u i}=1 & \forall u \in U \\
\sum_{u \in U} s_{u k} y_{u o j} \geq E_{k o} & \forall k \in K, o \in O, j \in J \\
\sum_{u \in U} y_{u o j} p_{u} \geq B_{o} & \forall o \in O, j \in J \\
\sum_{u \in U} z_{u} q_{u o j}=1 & \forall o \in O, j \in J
\end{array}
$$




$$
\begin{array}{ll}
r_{u i o j} \geq\left(x_{u i}+q_{u o j}-1\right) & \forall u \in U, o \in O, i \in I, j \in J \\
m_{u i o j} \geq\left(x_{u i}+y_{u o j}-1\right) & \forall u \in U, o \in O, i \in I, j \in J \\
F_{o j} \geq \sum_{i \in I} r_{u i o j}\left(t_{i j}+a_{u}\right) & \forall u \in U, o \in O, j \in J \\
T_{o j} \geq \sum_{i \in I} m_{u i o j}\left(t_{i j}+a_{u}\right) & \forall u \in U, o \in O, j \in J \\
F_{o j}, T_{o j} \geq 0 & \forall o \in O, j \in J \\
x_{u i}, y_{u o j}, q_{u o j}, r_{u i o j}, m_{u i o j} \in\{0,1\} & \forall u \in U, o \in O, i \in I, j \in J
\end{array}
$$

The objective function (1) of the model is the sum of a weighted combination of the time to the first response and the time to the full response multiplied by the expected number of accidents. The weight parameters, $\alpha$ and $\beta$, can be varied to obtain solutions with different characteristics.

Constraint (2) guarantees that each unit is located in exactly one zone. The requirement of units of different types to a certain type of accident ensures by Constraint (3). Constraint (4) ensures that the required number of firefighters responds to each accident. That there is a first responding unit to each accident is ensured by Constraint (5), and the constraint also ensures that the unit is permitted to make a first response.

Constraint (8) expresses the first response time for a certain accident in a certain zone. The first response time is the first responding unit's travel time plus the preparation time for the unit. Similarly, the full response time is the sum of the travel time and preparation time for the last arriving required unit. The full response time for a certain accident in a certain zone is presented by Constraint (9). Constraints (6) and (7) are supplementary constraints to Constraints (8) and (9). Constraint (10) ensures non negativity and Constraint (11) confirms binary variables.

\subsection{Solution Method}

The location model has been solved optimally by Gurobi Optimizer 5.6.0 by using a PC laptop, with an Intel Core i5-2520M, $2.50 \mathrm{GHz}$ processor, and $8 \mathrm{~Gb}$ RAM. However, optimal solutions have only been found for very small problems with limited number of units and demand zones. In an area with 30 zones, optimal solutions can be found within seconds for one or two units. However, the solution time depend on the type of units that are included as well as on the requested number of units and firefighters. Including three units, no optimal solution has been found within six hours. However, within six hours, feasible solutions could be found with a gap of $15 \%$ (including three units). For larger problems no optimal solutions have been generated by Gurobi. 
For larger problems, solutions are obtained using a reduced variable neighborhood search heuristic, (RVNS), which has been proved to be efficient for large p-median problems (Hansen et al, 2001). Variable neighborhood search, (VNS), is a metaheuristic for solving combinatorial and global optimization problems. The basic idea of VNS is to use a systematic change of neighborhoods combined with a local search algorithm. Assuming that a start solution can be found, a VNS would typically start with randomly selecting a new solution from one of the neighborhoods. Using this new solution as a starting point, a local search is used to find a local optimum. In the local search procedure, small local changes are made to find a better solution. When a local optimum is found in the current neighborhood, the process moves to a new neighborhood, and a new local search is made to find a local optimum in the new neighborhood. In the end, the best local optimum with respect to all neighborhoods is kept as the global optimum. Sometimes the local search procedure can be too costly. The search space can be very large and thus the local search procedure too time consuming. The reduced VNS (RVNS) is a simplified VNS, where the local search procedure has been removed in order to improve the search efficiency. Thus, in RVNS, the search procedure consists of searching for new solutions by a systematic change of neighborhoods. When a solution in a neighborhood is found and the new solution is better than the current solution, the search moves to the new solution, and a new random neighbor is selected from the neighborhoods. If the new solution is worse than the current solution, the current solution is kept, and the search iterates to a new neighborhood. A new neighbor is then randomly generated from the included neighborhoods. The search continues until a stop condition is satisfied (Hansen and Mladenović, 2001).

In the RVNS heuristic used to solve the developed model, three different neighborhood types, i.e. moves, for the resources are used in order to explore the solution space and find better solutions. Consequently, $\mathrm{k}_{\max }=3$. The neighborhood types that are used are: "interchange", "local interchange" and "swap", which results in a total of nine different neighborhoods. In the interchange move, one, two or three units are moved to new locations. In local interchange, one, two or three units are moved to new locations within a maximum predefined distance $d$, in travel time, from the units' current position. In the swap move, the locations of one, two or three pairs of units are swapped, i.e. one or several pairs of units swap location with another pair of units.

The method starts from an initial feasible solution, which from the beginning of the solution process is the best solution. During the solution process, given the current best solution $x$, a new candidate solution is randomly generated from one of the neighborhoods. If the new solution, $x^{\prime}$, is better than the current best found solution, $x$, the search moves to this solution (i.e. $x=x^{\prime}$ ). The same neighborhood structure is then used to generate a new candidate solution originating from the new best found solution, $x$. If $x$ ' is worse than $x$, the algorithm iterates to one of the other neighborhoods. The search continues for a pre-specified number of iterations. The pseudo code for the reduced variable neighborhood search procedure used for solving the model is presented in Table 4. 
Table 4. Reduced variable neighborhood search for locating fire and rescue resources.

The pseudo code for the Reduced Variable Neighborhood Search

Set $k=1$; neighborhood type $=1 ; x=x^{\prime}=$ the current best solution; $\mathrm{k}_{\max }=3 ; d=$ maximum travel time While (stop condition)

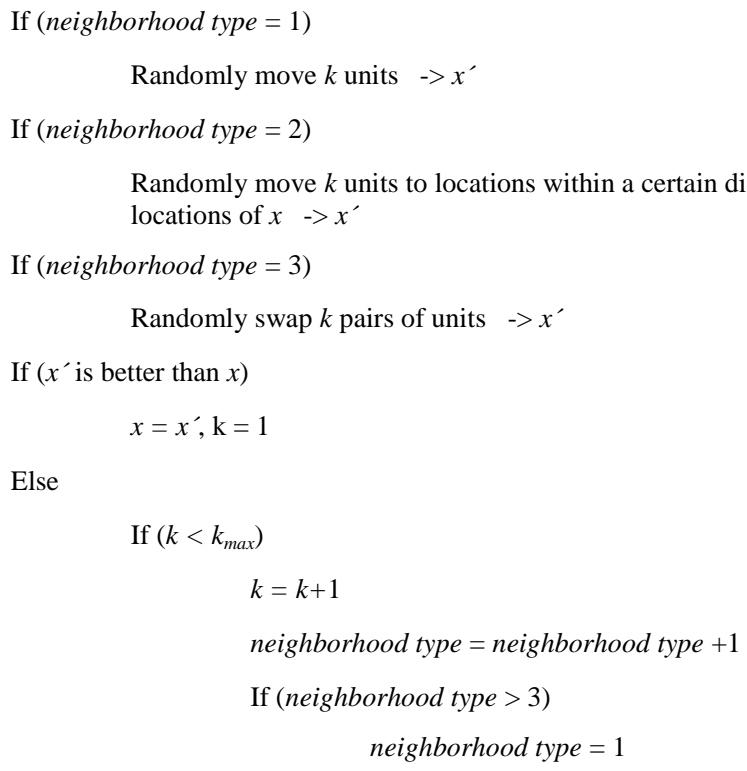

\subsection{Test Area}

The location problem has been solved for a test area. The test area is the Östergötland County in Sweden.

The fire and rescue service respond to many different types of accidents. However, the accidents in Sweden that cause the most serious injuries and losses of life are housing fires and traffic accidents (Norrköpings Brandförsvar, 2008). It is thus usually important to reach these types of accidents as quickly as possible and also to have resources suitably located so that they can make efficiently responses to these types of accidents. Consequently, the accidents that are considered for locating resources in the test area are housing fires and traffic accidents.

For urgent alarms, the initial phase is usually the most essential. It is desirable with a quick response time for the resources that are needed in the initial phase, and thus their locations are important. The location for back-up resources for prolonged operations and for non-urgent missions is in general of less importance. Consequently, it is only the first (initial) phase of the response that is modeled and, thus, the resources that initially are required for an incident 
that are included. For housing fires and traffic accidents, this means that base units, ladder units and small units are the included resources for Östergötland County.

Base units are used for all accidents and provide the base equipment needed to take care of an accident. Ladder units are primarily used for fires in high-rise buildings. Small units are basically used to perform first responses, but they can also be used to provide the requested staffing to any accident. The small units carry less equipment than the base units and can therefore only perform basic rescue work before the base unit has arrived. Since it is only the first phase of the response that is modeled, it is not taken into account that an accident may sometimes need special or additional resources or equipment.

In the county, there are 13 municipalities and together they have 23 base vehicles manned by four or five firefighters, six ladder vehicles manned by one firefighter and nine small vehicles manned by two or three firefighters. A unit is a combination of a fire vehicle and one or more firefighters. Furthermore, each unit also has a specific preparation time, which is the time from receiving an alarm until the unit leaves the station. This time varies between $90-360$ seconds depending on whether it is a full-time or a part-time unit. Full-time units have a preparation time of 90 seconds while part-time units have a preparation time of more than 90 seconds. A more detailed presentation of the number of the different types of units included in the county is presented in Table 5. For example, there are eight base units with five firefighters who have a preparation time of 90 seconds.

Table 5. The number of units in Östergötland County

\begin{tabular}{|c|c|c|c|c|c|}
\hline \multirow[b]{3}{*}{ Unit } & \multirow[b]{3}{*}{$\begin{array}{l}\text { Number of } \\
\text { firefighters }\end{array}$} & \multicolumn{4}{|c|}{ Number of Units in Östergötland County } \\
\hline & & \multicolumn{4}{|c|}{ Preparation Time (in seconds) } \\
\hline & & 90 & 240 & 300 & 360 \\
\hline \multirow{2}{*}{ Base Units } & 4 & \multirow{4}{*}{8} & \multirow{4}{*}{1} & 2 & 1 \\
\hline & 5 & & & 9 & 2 \\
\hline \multirow{2}{*}{ Small Units } & 2 & & & 2 & \\
\hline & 3 & & & 6 & 1 \\
\hline Ladder Units & 1 & 5 & & 1 & \\
\hline
\end{tabular}

The need for firefighters and units varies depending on the type of accident, i.e. housing fires or traffic accidents. To provide a full response to any accidents in Östergötland County, at least one base unit with five firefighters are required. In case of a fire in an area with high-rise buildings, a ladder unit with one firefighter is also requested. Thus, multiple units are sometimes needed for an accident to ensure the requirements of resources, both in terms of vehicles and firefighters.

A base unit is required for all considered accidents, and, thus, can respond to all accidents. First responses can only be provided by base units or small units. Small units can also be used to provide the required staffing (five or six firefighters) to achieve a full response. Ladder 
units can only be used for fires in high-rise buildings, i.e. they cannot be used to provide first responses or contribute with personnel resources to other types of accidents. A ladder unit must also be located together with at least one base unit. The information about how the resources can be used was provided from the fire and rescue service in Östergötland County.

To solve the problem for the Östergötland County, necessary input data consists of forecasts of the included accidents, expected travel times for the units and the location of high-rise buildings in Östergötland County. The forecasts are based on regression analysis-type models provided by Karlstad University for fire accidents and by the Swedish National Road and Transport Research Institute (VTI) for traffic accidents.

The forecasts were delivered in 250 meter squares, which resulted in approximately 45000 demand zones for Östergötland County. Both the forecasts for housing fires and traffic accidents indicate the expected number of accidents per zone and year. To be computationally tractable, the data is aggregated to 3000 demand zones by using the K-means clustering aggregation method (Erkut and Bozkaya, 1999). The forecasts have been validated against historical data in the Norrköping municipality obtained from Räddningstjänsten Östra Götaland. A positive correlation can be shown with the historical data for both housing fires and traffic accidents, and the conclusion is that the forecasts are good enough for the intended purpose. In total, the forecasts predict 270 fire accidents and 700 traffic accidents per year for the county.

Travel times from each demand zone to every other demand zone are provided by the Swedish agency for growth policy analysis. The travel times consist of shortest-pathcalculations, based on data from the National road database (NVDB) with speed ratings appropriated for emergency vehicles.

Fires in high-rise buildings can only be expected in areas where high-rise buildings can be found. In the County of Östergötland, high-rise buildings can be found in the five largest cities, i.e. Linköping, Norrköping, Söderköping, Mjölby and Motala. In addition to a base unit and five firefighters, a ladder unit and an extra firefighter are also needed for fires in high-rise buildings.

\subsubsection{Model Modifications}

To solve the location problem for Östergötland County, the developed location model has been adapted to the conditions in the area. There are 38 various units to locate and the area is divided into 3000 demand zones. Two types of accidents, housing fires and traffic accidents, are considered in addition to three types of units, base units, ladder units and small units. The included sets thus become as follows;

$O$ : the set of accident types $\in\{1,2: 1=$ fire accidents, $2=$ traffic accidents $\}$

$I, J:$ the set of zones $\in\{3000\}$

$U$ : the set of units $\in\{38\}$

$K$ : the set of unit types $\in\{1,2,3: 1=$ Base unit, $2=$ Ladder Unit, $3=$ Small Unit $\}$ 
In case of a fire accident in a high-rise building, a ladder unit and an extra firefighter are required. Therefore, a parameter that indicates if there are high-rise buildings in a zone is included in the model:

$h_{j}=\left\{\begin{array}{l}1 \text { if there are highrise buildings in zone } j \\ 0 \text { otherwise }\end{array}\right.$

A constraint that ensures that a ladder unit and an additional firefighter respond to fires in high-rise buildings is also included:

$$
\sum_{u \in U} s_{u k} y_{u o j} p_{u} \geq h_{j} \quad \forall o=\{1\}, k=\{2\}, j \in J
$$

Since a ladder unit must be located together with at least one base unit, the following constraint is included:

$$
x_{v i} s_{v l} \leq \sum_{u \in U} x_{u i} s_{u b} \quad \forall b=\{1\}, l=\{2\}, v \in U, i \in I
$$

Since the ladder unit can only be used to make responses to fire accidents in high-rise buildings, i.e. they cannot be used to contribute with personnel resources to other types of accidents. Therefore, Constraint (4) in the model is changed as follows;

$$
\sum_{u \in U} s_{u k} y_{\text {uoj }} p_{u} \geq B_{o} \quad \forall k=\{1,3\}, o \in O, j \in J
$$

\subsection{Test Scenarios}

The model has been solved for a number of scenarios with different conditions to produce suggestions on how the resources can be located in the Östergötland County. The area and the resources' current location are shown in Figure 7. Various scenarios are designed to evaluate how different aspects and characteristics affect the result when solving the problem. Examples of aspects and characteristics that are changed in the scenarios are how the resources can be used, the importance of a first and a full response, how the resources can be located and the number of available resources in the area. The location problem is solved for the scenarios to investigate if any improvements can be found compared to if the resources are located as in the current situation.

The scenarios are presented in Table 6. In the first three scenarios, the weight parameters are weighted differently. In Scenarios 4 and 5, a penalty fee is added if a unit locates away from its original location. It is a penalty fee that depends on the distance the unit is moved. The penalty fee is added in order to avoid moves that are too costly.

For Scenarios 4 and 5, the following adjustments have to be made:

1. Let $W(u) \in I$ be the zone where unit $u$ is originally located. 
2. Let $c=$ the penalty fee added to the distance (in seconds) if a unit is located away from its original location

3. Add the following to the objective function of the model: $\left(-c \sum_{u \in U} \sum_{i \in I} t_{W(u) i} x_{u i}\right)$

For Scenario 6, the restrictions on the ladder units are removed, i.e. the ladder units can be used for deployment to any accidents, and they do not need to be located together with a base unit. That means, Constraints (II) and (III), which are described in the previous section, should not be included in the model.

Table 6. Descriptions of the scenarios.

\begin{tabular}{|c|c|}
\hline Scenario & Description \\
\hline Current & The resources are located as they are in the current situation. \\
\hline Scenario 1 & $\alpha=0.9$ and $\beta=0.1$ \\
\hline Scenario 2 & $\alpha=0.1$ and $\beta=0.9$ \\
\hline Scenario 3 & $\alpha=0.7$ and $\beta=0.3$ \\
\hline Scenario 4 & $\begin{array}{l}\alpha=0.7 \text { and } \beta=0.3 \\
c=0.1\end{array}$ \\
\hline Scenario 5 & $\begin{array}{l}\alpha=0.7 \text { and } \beta=0.3 \\
c=1\end{array}$ \\
\hline Scenario 6 & $\begin{array}{l}\alpha=0.7 \text { and } \beta=0.3 \\
\text { The ladder unit can respond to any type of accident, be used as a first response and does not } \\
\text { need to be located in tandem with a base unit. }\end{array}$ \\
\hline Red 1 & $\begin{array}{l}\alpha=0.7 \text { and } \beta=0.3 \\
\text { This scenario contains a reduced number of resources. Two base units, one ladder unit and } \\
\text { three small units are removed compared to the current situation. }\end{array}$ \\
\hline
\end{tabular}

\subsection{Computational Results and Discussion}

The scenarios described in the previous section are solved by using the reduced variable neighborhood search heuristics described in Section 4.2. $K_{\max }$ is set to 3, since initial testing showed that using a larger value will increase the computational times without giving a better result. For the neighborhood type 2, the local interchange move, the maximum distance $d$ in travel time from the units' current position is set to 500 seconds. The algorithm has been run using a HP ProLiant DL385. For each scenario one million iterations have been run, which takes about three to four hours. The location problem has been solved once for each scenario. When using stochastic methods, a number of solutions are usually produced for variations of the problem to evaluate the method. But, since focus is to produce and evaluate solutions to various types of scenarios with different characteristics, the solution method has not been further evaluated. 
Table 7. Computational results

\begin{tabular}{lccccccc}
\hline Scenario & $\boldsymbol{\alpha}$ & $\boldsymbol{\beta}$ & $\begin{array}{c}\text { Penalty } \\
\text { fee - }\end{array}$ & $\begin{array}{c}\text { Obj } \\
\text { function }\end{array}$ & $\begin{array}{c}\text { FiR mean } \\
\text { time }\end{array}$ & $\begin{array}{c}\text { FuR mean } \\
\text { time }\end{array}$ & Improvement \\
\hline \hline Current & 0.9 & 0.1 & & 502529 & 514 & 556 & - \\
Scenario 1 & 0.9 & 0.1 & & 396922 & 406 & 440 & $21 \%$ \\
Scenario 2 & 0.1 & 0.9 & & 419261 & 412 & 435 & $22 \%$ \\
Scenario 3 & 0.7 & 0.3 & & 402170 & 406 & 435 & $21 \%$ \\
Scenario 4 & 0.7 & 0.3 & 0.1 & 409728 & 409 & 439 & $20 \%$ \\
Scenario 5 & 0.7 & 0.3 & 1 & 430141 & 422 & 458 & $16 \%$ \\
Scenario 6 & 0.7 & 0.3 & & 381064 & 365 & 458 & $25 \%$ \\
Red 1 & 0.7 & 0.3 & & 436657 & 440 & 474 & $22 \%$ \\
\hline
\end{tabular}

The computational results for each scenario are presented in Table 7. $\alpha$ and $\beta$ are the weight parameters for the first and full responses, respectively. They indicate how the time for the first response and the time for the full response will affect the objective function value. It may be natural to use the same weight (0.5) for the response times for both full and first responses. However, this makes the model automatically promote full responses, because the time for the full response is always higher than or at least as high as for a first response. By using a slightly higher weight for the first response, the response times' influence becomes well balanced. The penalty fee $c$ is a cost that is placed on the resource's distance in seconds from its original location. The cost increases with the distance. The Obj function is the objective function value of the solution. FiR mean time is the mean time for a first response to reach an accident and FuR mean time is the mean time for a full response to reach an accident within the area. The column Improvement states the solution's improvement as a percentage compared to if the same scenario is evaluated for the current situation, i.e. the resources are located as they are in the current situation. The mean times are presented in seconds. 
Table 8. Degree of coverage

\begin{tabular}{lcccccc}
\hline Scenario & FiR 10 min & $\begin{array}{c}\text { FuR 10 } \\
\text { min }\end{array}$ & Area 10 min & FiR 20 min & FuR 20 min & Area 20 min \\
\hline \hline Current & $74 \%$ & $64 \%$ & $33 \%$ & $98 \%$ & $96 \%$ & $89 \%$ \\
Scenario 1 & $84 \%$ & $78 \%$ & $37 \%$ & $98 \%$ & $97 \%$ & $89 \%$ \\
Scenario 2 & $83 \%$ & $81 \%$ & $37 \%$ & $98 \%$ & $97 \%$ & $88 \%$ \\
Scenario 3 & $84 \%$ & $79 \%$ & $38 \%$ & $98 \%$ & $97 \%$ & $89 \%$ \\
Scenario 4 & $83 \%$ & $80 \%$ & $39 \%$ & $98 \%$ & $97 \%$ & $90 \%$ \\
Scenario 5 & $81 \%$ & $78 \%$ & $38 \%$ & $98 \%$ & $96 \%$ & $89 \%$ \\
Scenario 6 & $87 \%$ & $79 \%$ & $42 \%$ & $99 \%$ & $97 \%$ & $90 \%$ \\
Red 1 & $81 \%$ & $76 \%$ & $32 \%$ & $97 \%$ & $96 \%$ & $84 \%$ \\
\hline
\end{tabular}

Table 8 presents the degree of coverage of the accidents in the area for different response times as well as the coverage of the county's area within different times. FiR $10 \mathrm{~min}$ states the proportion of the accidents that can be reached with a first response within 10 minutes while FuR 10 min states the proportion of the accidents that can be reached with a full response within 10 minutes. Area $10 \mathrm{~min}$ shows the proportion of the county's area that can be reached by any response unit within 10 minutes. FiR $20 \mathrm{~min}$, FuR $20 \mathrm{~min}$ and Area $20 \mathrm{~min}$ states the same as the items just declared, but for a response time of 20 minutes.

The results of the scenarios' produced solutions indicate that it is possible to improve the response times to expected accidents in the county of Östergötland by up to $25 \%$ compared to if the resources are located as today. The results for Scenarios 1 and 2 clearly show that the mean times for first and full responses depend on how the responses are weighted. When comparing Scenarios 1 and 2, it appears that when focusing on the first responses (Scenario 1) the model wants to reduce the first response time, while focusing on the full responses (Scenario 2) the model wants to reduce the full response time. The selection of parameter settings for $\alpha$ and $\beta$ in Scenario 3 has been proven to be a good balance between a first and a full response ( $\alpha=0.7$ and $\beta=0.3$ ). The settings are attractive and reasonable from a user point of view, and Scenario 3 produces very competitive results when studying the mean response times and the coverage results. 


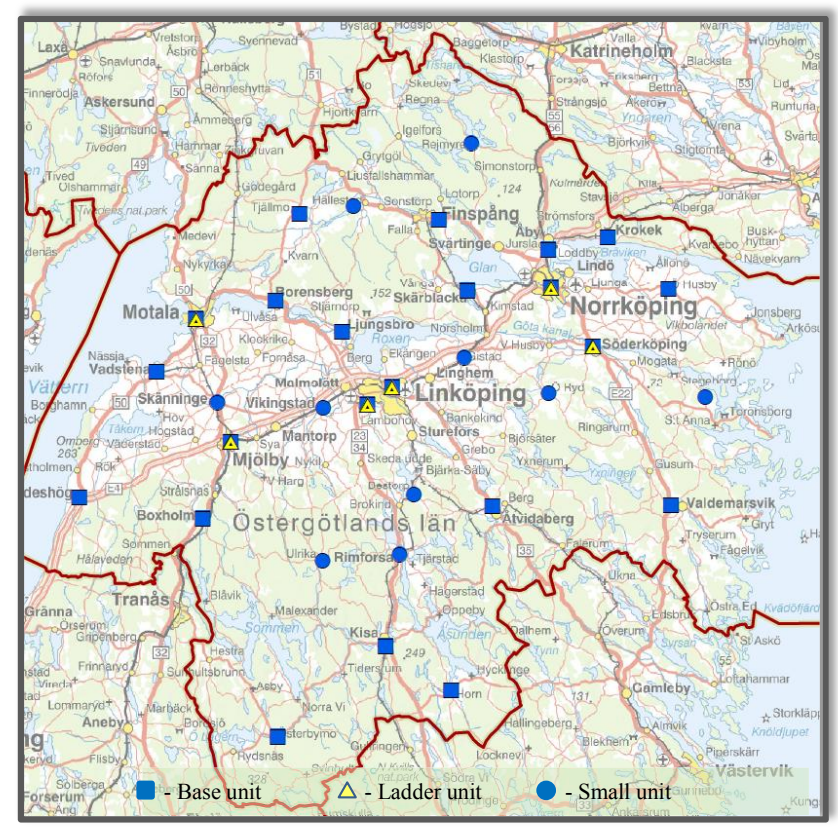

Figure 7. The resources' present locations in Östergötland County

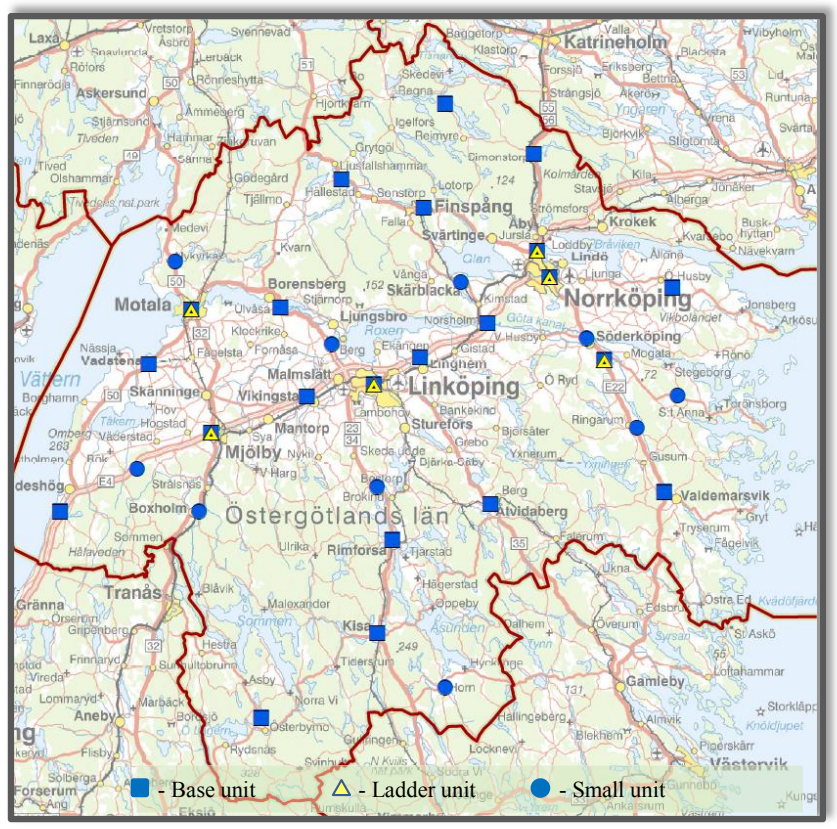

Figure 8. The resources' suggested locations in Scenario 3.

For the studied area, i.e. Östergötland County, the resources' current location and the resources' suggested locations in Scenario 3 are visualized in Figure 7 and Figure 8. The main 
changes between the locations in the current situation and the suggested locations in Scenario 3 are that the units are located closer to main roads (to high-risk areas for traffic accidents). For example, two base units are located along the highway between Norrköping and Linköping (which are the two largest cities in the county) compared with one small unit in the current situation. In the produced solution, some of the base units have also moved closer to the larger cities, while some of the small units have moved further out in the countryside or been placed where they more easily can make quick first responses.

Another noticeable change from the current situation is that one of the ladder units, currently located in Linköping, has moved to Norrköping in the produced solution. Furthermore, one of the base units, also currently located in Linköping, has changed location and is in the produced solution placed along the highway a bit outside the city. Still, it is notable that even though the two solutions look quite alike, the expected response times have decreased by approximately $20 \%$. One could suspect that the similarity between the two solutions is due to the fact that the resources' present locations are used as the start solution. However, similar locations are obtained even when using randomized start solutions.

When a penalty fee is added to the distance between the resources' suggested locations and their current location, as in Scenarios 4 and 5, the solutions are as expected not as good as without the penalties. The results from these scenarios, when the resources are forced to be located closer to their current positions, are, however, interesting from a practical point of view. Since the penalty fee makes some moves too costly, only the most beneficial changes will be implemented. Thus, the penalty fee gives solutions where many resources keep their old location, which makes the solution easier to implement from a practical point of view. And the improvement in response times and coverage are significant.

In Scenario 6, the ladder units can be used as resources for any type of accident. They can also be used as first responses and they do not need to be located together with a base unit. It means that the ladder units can be utilized just as small units. This gives the model incentive to focus more on the first response component in the objective function, i.e. the first response time. The result shows a high coverage for the first response and the lowest mean response time for first responses, while the mean response time for full responses are a bit higher compared to the other solutions produced by the model. The motivation for this is that the model utilizes the ladder units as first responses and also to complement base units with less than five firefighters. The results also show that the ladder units are located closer to high-risk areas for traffic accidents rather than locating them close to high-rise buildings. This may be due to the fact that the number of traffic accidents and other housing fire accidents vastly outnumber the expected fire accidents in high-rise buildings.

In Scenario Red 1, where the units are reduced by six (to 32 units) from the current situation (38 units), the results show that the units' location do not differ much from the units location in Scenario 3, except from those that are removed. Even when a significant amount of resources are removed from the area, the model is able to produce results that are superior to the current situation. The response times are improved by more than $14 \%$. 
The results in Table 8 show that all the scenarios' produced solutions provide a better coverage within 10 minutes compared to the current location. Hence, a larger proportion of the accidents can be reached within 10 minutes with both first and full responses. The produced results regarding the coverage do not differ very much among the scenarios apart from Scenario 6, where a first response can reach $87 \%$ of the expected accidents and $42 \%$ of the county's area can be reached within 10 minutes. In the other scenarios, a first response reaches in general around $83 \%$ of the expected accidents and around $37 \%$ of the county's area can be reached within 10 minutes. In the case of 20 minutes, the results show that the fire and rescue services in Östergötland already have a good coverage within 20 minutes. $98 \%$ of the accidents can be reached by a first response, $96 \%$ by a full response and $89 \%$ of the county's area can be reached within 20 minutes. Since the coverage within 20 minutes already in the current situation has proven to be at a good level, the produced solutions have not been able to show any significant improvements in that respect.

\subsection{Conclusions}

By using the developed model for locating resources, the results show that the response times to housing fires and traffic accidents can be reduced by around $20 \%$. Furthermore, a significantly larger proportion of the accidents can be reached within 10 minutes compared to the current location of resources. In the produced solutions, the units are located slightly closer to high-risk areas for traffic accidents. But in many cases, the locations suggested by the model are also similar to the units' current locations. Although, if allowed, the model will utilize the ladder units for first responses and to complement base units with less than five firefighters rather than placing the ladder units close to areas with high-rise buildings.

In the model, there is no prioritization between fire accidents and traffic accidents; it is just as important to quickly reach a traffic accident as it is to reach a fire accident. Since the traffic accidents outnumber the fire accidents in the input data by a factor 2.6, it becomes natural for the model to partially focus on locating resources close to high-risk areas for traffic accidents, i.e. the main roads in the county. However, fire accidents are in general more costly than traffic accidents, which is why it may be interesting to also weight the response times for the different types of accidents in order to find more balanced solutions regarding the different types of accidents. 


\section{Preparedness for Fire and Rescue Services}

In this chapter, the term preparedness in the context of the fire and rescue service in Sweden is described. This section clarifies how the fire and rescue service currently estimates preparedness and defines the main indicators that affect the preparedness. Considering the indicators, a measure in order to quantitatively determine the preparedness for the fire and rescue service is developed. The purpose of the measure, i.e. to quantitatively determine the preparedness, is to support the fire and rescue service with a more efficient basis for decision making and resource planning.

To use the developed measure, the resources that contribute to the preparedness must be known. Therefore, an optimization model for solving the resource selection problem has been developed. The problem has been solved both exactly and by a developed greedy algorithmbased heuristic for different situations. The heuristic is explained and solutions obtained from the heuristic are compared to optimal solutions in order to validate its utility.

Further, the preparedness measure has been validated and calibrated in order to identify thresholds that specify if the measured value indicates good or inadequate preparedness. The validation and calibration process was performed using preparedness evaluations conducted by experts from the Swedish fire and rescue service. The evaluations were compared to results calculated by the measure.

The chapter, thus, describes the entire development and validation process of the preparedness measure, and it also gives examples of how the measure can be utilized by the fire and rescue service.

\subsection{Preparedness}

The word preparedness is commonly used in the fire and rescue service. Preparedness describes the ability to respond to accidents at present and in the future. It is an estimation of how well prepared the fire and rescue service is to manage a certain type of accident. The fire and rescue service usually estimates the preparedness in order to plan their daily activities. It is not obvious how to measure preparedness, determine the factors that affect the preparedness, or to identify what can be considered good or poor preparedness. You will probably get different answers from different professionals depending on their individual points of view, experience and personality traits.

According to the fire and rescue services in Sweden, preparedness is useful in estimating urgent incidents, because these events are unplanned, i.e. they include accidents that can occur anywhere at any time. It is these kinds of accidents for which the fire and rescue service must be able to maintain preparedness. However, the fire and rescue service is not able to maintain good preparedness for all types of emergencies. Accidents that rarely occur or require very specialized resources, or more extensive and resource-consuming incidents, are too costly to continuously prepare for. Accidents that the fire and rescue service should be able to handle and be prepared for at all times are, rather, the more frequent everyday accidents that often occur and cause significant damage. The Swedish fire and rescue services are, for example, particularly interested in maintaining adequate preparedness for building fires and traffic 
accidents, since these are the accidents that cause most of the serious injuries and loss of life in Sweden (MSB, 2013).

When the fire and rescue service estimates the preparedness for an accident, it is usually the resources that are needed in the first phase that are considered because these are so integral to an emergency response operation. The initial phase is also generally the most critical in order to reduce loss of life and property damage (Björnberg and Melin, 2003). The initial phase of an accident is also something that the fire and rescue services in Sweden have recently started to place more focus on. This is discussed in more detail in Chapter 2. The fire and rescue service does not usually maintain preparedness for more extensive or resource-intensive incidents, or incidents that need special resources.

If the preparedness could be quantified and measured, it should be possible to effectively determine the specific preparedness for handling a certain type of accident. The preparedness can then be used to get an effective basis for decision making and planning of fire and rescue resources. The preparedness value can for example be used to get a sufficient overview of the whole response area, which is appropriate to be able to make quick and adequate decisions when planning for emergency incidents. If the preparedness is known, it is also possible to quickly and effectively be able to determine if there are enough resources to handle a particular type of accident. For example, if the preparedness has degraded in an area, it might be necessary to call for additional resources, or to relocate resources from other positions closer to the affected area. A clear overview of the preparedness may facilitate the planning process in such situations and also make it easier to revise plans and decisions if that are necessary.

Previously, no measurements have been developed in order to quantitatively measure the preparedness for the fire and rescue service. The fire and rescue services in Sweden estimate today the preparedness for different types of accidents by various rules of thumb according to their own organization's regulations and guidelines. They have currently no support system or support tool in order to estimate or measure the preparedness.

\subsubsection{Utilization of the Preparedness}

If the preparedness can be quantified, it should be possible to measure the preparedness and use the obtained value to achieve a more efficient planning of fire and rescue resources. The preparedness can for example be measured for various positions or zones in an area. If the preparedness then can be graded in order to specify if it is good, acceptable or inadequate, it would be possible to give a dispatcher a good view of the preparedness for a particular type of accident in the area. With a good overview of the preparedness, the dispatcher can determine how prepared the fire and rescue service is to respond to an accident, and also if any actions must be made to maintain a good level of preparedness.

An example of how the preparedness can be graded and visualized is showed in Figure 9. In the figure, an area is divided into squares and the preparedness for a certain accident is measured for each of these squares. The large squares, numbered 29, 23 and 21, represent fire stations in the area. When the preparedness is measured, the squares are colored in different 
colors depending on the value of the measured preparedness in each square. Green represents good preparedness. The orange squares represent acceptable preparedness, while red squares represent inadequate preparedness.

Such a map provides a visual overview of the preparedness and it can be used to present the current situation in an area, and it may facilitate the allocation and coordination of fire and rescue resources. The resources in the area could also preferably be visualized in the map in order to know what type of resources that currently are available and where the resources currently are located. If conditions change, the preparedness can also easily be re-measured, and the user will get a new visualization of the preparedness. Such a support would therefore possibly be valuable in the daily operational planning process when conditions often change and quick decisions must be made.

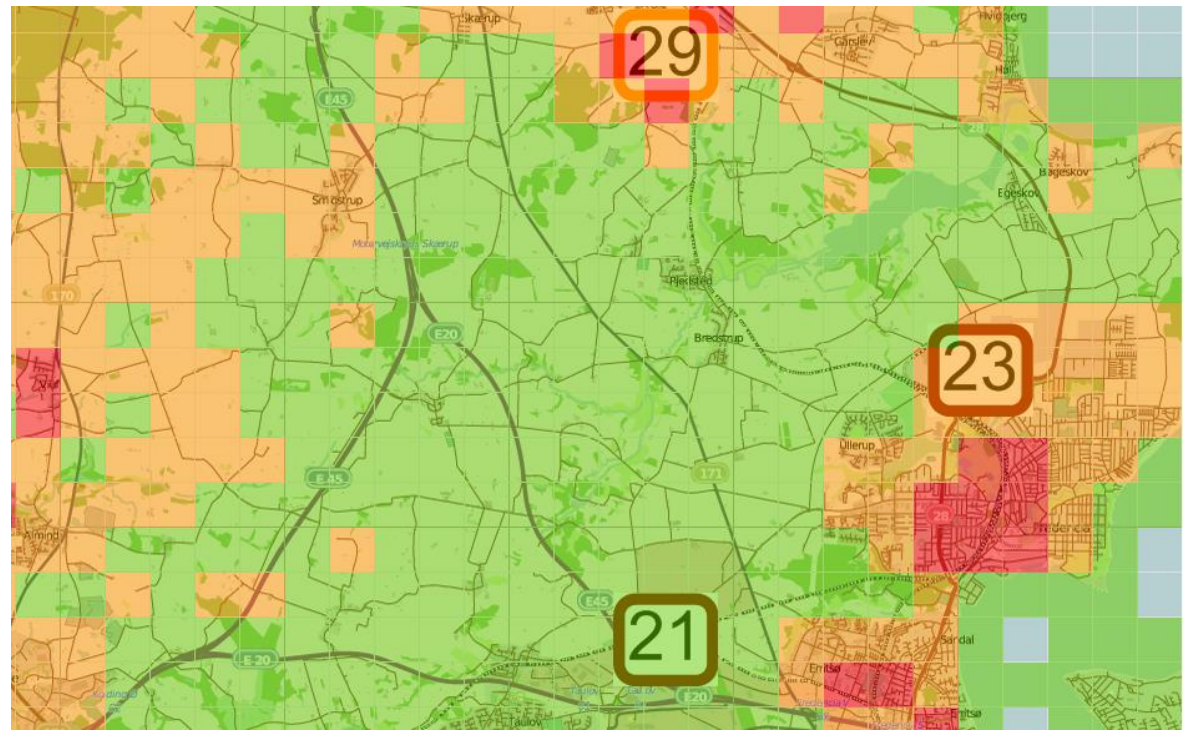

Figure 9. Example of how the preparedness can be visualized

It might also be of importance to have a sufficient overview of the preparedness when resources are divided into smaller groups. When resources are spread out at different places and not only located at fire stations, as discussed in Chapter 2, it becomes a tougher challenge for the dispatcher to get an adequate overview of the situation and all the resources, i.e. how the various teams of firefighters and fire officers are composed and where all the resources currently are located. If the dispatcher continuously can visualize the preparedness and all the resources in the response area, it is possible to visually identify the current situation and whether any type of resource is missing or if any resource has to be relocated. By being able to measure and visualize the preparedness, it may give the dispatcher good support in many important decisions in the planning process. They can for example determine whether the preparedness has deteriorated to an unacceptable level in any region, and if so, the dispatcher can utilize the preparedness visualization to make potential actions to improve the preparedness level. With such a support tool, it should also be possible to visualize how the 
preparedness would change if the dispatcher makes different actions. The dispatcher can thus make various tests before the real decisions must be made. For example, the dispatcher can visualize the preparedness both before and after a specific relocation of resources, and then decide if the resources should be relocated or not.

\subsubsection{Preparedness Estimation and Indicators that affect the Preparedness}

According to the fire and rescue services in Sweden, one of the main indicators for estimating preparedness is the availability of resources that are able to respond to upcoming incidents. The preparedness for a particular type of accident in a specific area is for example considered better the quicker the resources required for handling the accident can reach the area. The preparedness is thus related to the required resources response times and is therefore considered to deteriorate with an increase of the response times. Accordingly, to estimate the preparedness for an accident, the resources required to handle the specific accident must be known. To handle an accident, an amount of resources are required, which usually varies depending on the specific accident.

The preparedness is also directly affected by the demand for fire and rescue service, i.e. the number of accidents that is expected to occur in an area that require fire and rescue resources. Fire stations are therefore normally located in populated areas or close to populated areas to be able to reach the areas where accidents normally occur. It is typically considered better to quickly be able to reach areas where the expected demand is high, than to quickly be able to reach areas where accidents rarely occur. Thus, it may likely be acceptable with a slightly longer response time when the expected demand of fire and rescue service is considered low. For example, if the preparedness is estimated in two areas with different expected demand, the response time to the area with the lower expected demand can thus be longer than to the area with the higher expected demand, even if the same preparedness is obtained in both areas. 


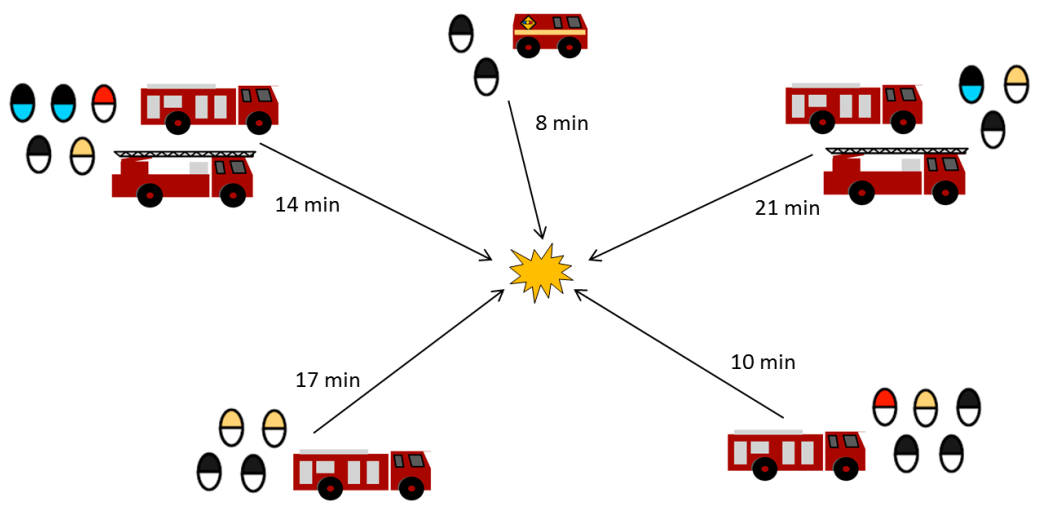

Resources

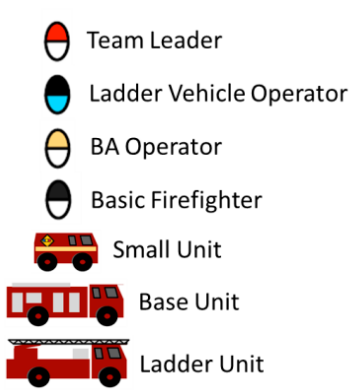

Figure 10. Example of how available resources can respond to an incident and contribute to the preparedness

Consider the example in Figure 10. Different resources are available to respond to an accident and the resources can reach the accident in the figure within different response times. All the resources are thus available to contribute to the preparedness for the given accident. As already mentioned, the preparedness is considered better in a certain area the quicker the required resources can reach the area. Also, if not all the requested resources can reach the area within the same time, it is normally considered better if a few resources can reach the area for example within 8 minutes and the remaining within 12 minutes, than if all can arrive within 10 minutes, as discussed in Chapter 2. All resources' individual response time is thus essential and considered when estimating the preparedness. However, according to the fire and rescue service in Sweden, they cannot say if it is preferable that a particular type of resource arrive quicker than another type. Thus, for handling an emergency, all required resources have the same importance, whether it regards different types of firefighters or fire vehicles. The main indicators that affect the preparedness are presented in Table 9.

Table 9. The main indicators that affect the preparedness

\begin{tabular}{ll}
\hline Indicator & How it affect the preparedness \\
\hline \hline Available resources & $\begin{array}{l}\text { To estimate the preparedness for a specific accident, the required } \\
\text { resources must be available in order to respond to emergencies. }\end{array}$ \\
Response time & $\begin{array}{l}\text { The preparedness is usually considered better the quicker the } \\
\text { required resources can reach the specific accident site. } \\
\text { The preparedness normally gets worse if the demand increase. }\end{array}$ \\
\hline
\end{tabular}

When fire and rescue services estimate the preparedness for a specific area or position, they estimate it for the current situation. When situations and conditions are changing, the preparedness may also change. The fire and rescue services must therefore always be 
observant towards changes in the operation in case they have to reorganize and repair poor preparedness.

\subsubsection{Quantification of Preparedness}

One way of quantifying the preparedness could for example be to consider the indicators that are found to be the most relevant for the fire and rescue service when they estimate the preparedness. The indicators that directly seem to affect the preparedness are defined as the forecast of the expected demand of service and the average response time for the resources that are required to handle a specific type of accident. When estimating the preparedness for a specific type of accident, it is thus the resources with the shortest response time that are required to handle the specific accident that also contribute to the preparedness.

If the preparedness should be quantified for a specific type of accident for the situation in Figure 10, the requested resources for handling such an accident must first be considered. Assume that the requested resources for the accident in this example are one fire vehicle, one ladder vehicle, one team leader, four basic firefighters, two ladder vehicle operators and one BA (Breathing Apparatus) team leader, i.e. a total of ten resources. The resources that will contribute to the preparedness for the specified accident would then be selected regarding to the available resources response times. It is the resources with the shortest response time that are contributing to the preparedness. The resources will thus be selected as follows: two basic firefighters can arrive within eight minutes; the team leader, two basic firefighters, the BA operator, and the base vehicle can arrive within ten minutes; and the remaining two required ladder vehicle operators and the ladder vehicle can arrive within fourteen minutes. If the expected demand for the specific type of accident also is known (for this example it is assumed to be 1.6 accidents per year) the preparedness can be quantified by multiplying the expected demand with the average response time for the contributing resources. The expression to measure the preparedness $P$, for the specific accident in Figure 10, becomes as follows:

$$
P=1.6\left(\frac{2 * 8+5 * 10+3 * 14}{10}\right) \approx 17.28
$$

The preparedness value itself does not express if the preparedness is adequate or not. To be able to interpret the preparedness value, the value must thoroughly be tested, calibrated and validated. It should however be noted that a low preparedness value corresponds to better preparedness. The preparedness value will probably be interpreted differently depending on the type of accident, situation, fire and rescue service organization, response area or country the preparedness is measured for. Also the unit of the forecast for the expected demand can affect the interpretation of the preparedness value. For example, the preparedness value might be interpreted differently if the number of expected accidents is 3 per year than if it is 3 per week. It is therefore important and necessary to systematically calibrate and validate the preparedness value for each specific situation to be able to determine if the preparedness value indicates good or poor preparedness.

During the development of the expression to measure the preparedness, there has been continuous communication with the fire and rescue service in Sweden in order to ensure an 
adequate quantification of the preparedness. During the process, fire and rescue service experts found that the expected demand factor had too much impact on the preparedness in relation to the response time. For a very low expected demand, the preparedness measure indicated a good level of preparedness even if the response time was set to several hours, while for a regular expected demand the preparedness measure indicated an extremely bad preparedness even if the response time was set to only a few minutes. To solve that problem and in order to get a more balanced measure, a parameter $\gamma$ was added to the expected demand factor. The expression to measure the preparedness $P$, for the specific accident in Figure 10, will then be revised as follows:

$$
P=1.6^{\gamma}\left(\frac{2 * 8+5 * 10+3 * 14}{10}\right) \approx 12.15 \quad(\gamma=1 / 4)
$$

Various parameter settings of $\gamma$ were tested together with the fire and rescue service. Finally, for the input data used in this thesis, given that the demand is expressed as number of accidents per year, an adequate preparedness measure was found when $\gamma=1 / 4$.

\subsubsection{Formulation of the Preparedness Measure}

The quantified preparedness value is intended to be used to indicate how prepared the fire and rescue service is for responses to a specific type of accident in a particular position or zone in an area. The preparedness measure was developed for application to multiple emergency circumstances. That also means it should be possible to use the measure to calculate the preparedness for any type of accident. The general expression for calculating the preparedness for handling a particular accident of type $a$ in a specific zone $j$ can then be formulated as follows:

$$
P_{a j}=d_{a j}{ }^{\gamma} \frac{F_{a j}}{N_{a}} \quad \forall a \in A, j \in J
$$

where $F_{a j}$ is the total response time for the requested resources that contribute to the preparedness for accidents of type $a$ in zone $j . N_{a}$ is the number of resources that are needed to handle the accident $a$, i.e. the requested resources that contribute to the preparedness, and $d_{a j}$ is the expected number of accidents of type $a$ in zone $j . \gamma$ is a parameter related to the expected demand factor.

Since the requested resources consist of both firefighters and fire vehicles, the total response time $F_{a j}$ for the requested resources can be expressed as follows:

$$
F_{a j}=\sum_{p \in P} V_{p a j}+\sum_{u \in U} T \quad \forall a \in \in \in, j \in J
$$

where $V_{p a j}$ is the response time for a requested personnel resource $p$ that contributes to the preparedness for handling accidents of type $a$ in zone $j . T_{u a j}$ is the response time for a requested vehicle $u$ that contributes to the preparedness for handling accidents of type $a$ in zone $j$. 
As mentioned, a low value indicates better preparedness. It may, however, be argued that good preparedness reflects a high value, and indeed it is possible to construct a measure that has that quality, although at a risk of introducing non-linearity. However, since the two main components in (A), the resources' response times and the expected demand of service, improve the preparedness when decreasing, the preparedness value $P_{a j}$ also share that feature.

When measuring the preparedness, the contributing resources should be selected so that the response time of deployment of fire and rescue resources is minimized, i.e. in order to minimize the resources' total response time $F_{a j}$. The response time should be minimized because it is always the requested resources with the shortest response time that contribute to the preparedness. Since the personnel may have multiple skills, e.g. a firefighter might be able to both work in smoke-filled spaces as well as manage ladder vehicles, but can only utilize one skill at a time, it is not always obvious how the resources should be selected. For that reason, to be able to select the most suitable available resources, a mathematical resource selection model has been developed; see Section 5.2. The model minimizes the required resources' response times. This also means that the preparedness $P_{a j}$ will be minimized. Thus, in order to measure the preparedness by using (A), include also solving the resource selection model presented in Section 5.2.

Measuring the preparedness requires, besides access to the available resources, access to forecasts for the type of accident for which the preparedness should be measured. The forecast should estimate the number of accidents expected to occur in a specific area at a certain time or within a certain time window. Forecasts may vary over time, which makes it important to also ensure that the user have forecasts for the desired period of time or moment the preparedness should be measures for.

\subsection{A Resource Selection Model}

To be able to determine the most suitable available resources that are requested in order to measure the preparedness, a resource selection model is developed and presented in this section. The requested resources are selected by the model from the resources that are available depending on their geographical locations and capabilities to respond to accidents. The model can, thus, be used to select appropriate resources to any type of accident, without necessarily measuring the preparedness.

The model minimizes the response time for the resources that are requested (in order to measure the preparedness) for a given type of an accident in a certain position or zone in an area. The model can be used for any type of demand for different types of vehicles and personnel competencies, given that the requested types of resources are available. The model selects resources for a given accident in a specific zone and it is formulated as follows:

Sets:

$U=$ the set of vehicles

$P=$ the set of persons

$K=$ the set of vehicle types

$R=$ the set of personnel competencies 


\section{Variables:}

$x_{p r}=\left\{\begin{array}{l}1, \text { if person } p \text { contributes with competency } r \\ 0, \text { otherwise }\end{array}\right.$

$y_{u}=\left\{\begin{array}{l}1, \text { if vehicle } u \text { contributes to the specific accident } \\ 0, \text { otherwise }\end{array}\right.$

$z_{p u}=\left\{\begin{array}{l}1, \text { if person } p \text { is assigned to vehicle } u \\ 0, \text { otherwise }\end{array}\right.$

$f_{p u}=\left\{\begin{array}{l}1, \text { if person } p \text { drives vehicle } u \\ 0, \text { otherwise }\end{array}\right.$

$V_{p}=$ the response time for person $p$ (if the person is requested)

$T_{u}=$ the response time for vehicle $u$ (if the vehicle is requested)

\section{Parameters:}

$E_{p u}=\left\{\begin{array}{l}1, \text { if person } p \text { can be assigned to vehicle } u \\ 0, \text { otherwise }\end{array}\right.$

$G_{p u}=\left\{\begin{array}{l}1, \text { if person } p \text { can drive vehicle } u \\ 0, \text { otherwise }\end{array}\right.$

$Q_{p r}=\left\{\begin{array}{l}1, \text { if person } p \text { posesses competency } r \\ 0, \text { otherwise }\end{array}\right.$

$S_{u k}=\left\{\begin{array}{l}1, \text { if vehicle } u \text { is of type } k \\ 0, \text { otherwise }\end{array}\right.$

$W_{u}=$ the number of seats in vehicle $u$

$t_{u}=$ travel time for vehicle $u$

$a_{p}=$ preparation time for person $p$

$B_{r}=$ the demand for persons with competency $r$

$D_{k}=$ the demand for vehicles of type $k$

Minimize $\sum_{u \in U} T_{u}+\sum_{p \in P} V_{p}$

Subject to

$$
\begin{array}{ll}
\sum_{p \in P} x_{p r} \geq B_{r} & \forall r \in R \\
\sum_{u \in U} S_{u k} y_{u} \geq D_{k} & \forall k \in K \\
\sum_{p \in P} z_{p u} \geq y_{u} & \forall u \in U
\end{array}
$$




$$
\begin{aligned}
& \sum_{p \in P} z_{p u} \leq W_{u} \sum_{p \in P} f_{p u} \quad \forall u \in U \\
& \sum_{u \in U} z_{p u} \geq \sum_{r \in R} x_{p r} \quad \forall p \in P \\
& \sum_{u \in U} z_{p u} \leq 1 \quad \forall p \in P \\
& \sum_{r \in R} x_{p r} \leq 1 \quad \forall p \in P \\
& x_{p r} \leq Q_{p r} \quad \forall p \in P, r \in R \\
& z_{p u} \leq E_{p u} \quad \forall p \in P, u \in U \\
& \sum_{p \in P} f_{p u} \leq 1 \quad \forall u \in U \\
& \sum_{u \in U} f_{p u} \leq 1 \quad \forall p \in P \\
& f_{p u} \leq z_{p u} \quad \forall p \in P, u \in U \\
& f_{p u} \leq G_{p u} \quad \forall p \in P, u \in U \\
& T_{u} \geq\left(t_{u}+a_{p}\right)\left(z_{p u}+y_{u}-1\right) \quad \forall u \in U, p \in P \\
& V_{p} \geq t_{u}\left(z_{p u}+\sum_{r \in R} x_{p r}-1\right)+ \\
& a_{q}\left(z_{p u}+\sum_{r \in R} x_{p r}+z_{q u}-2\right) \\
& \forall p, q \in P, u \in U \\
& \forall p \in P, r \in R, u \in U, k \in K \\
& \forall p \in P, u \in U
\end{aligned}
$$

The objective function (1) is the sum of the response times for all vehicles and all persons that are selected to contribute to the preparedness in a certain zone in an area for a specified type of accident. To select the most appropriate resources, the response times for the requested resources are minimized.

Constraint (2) in the model ensures the minimum manning level of personnel competencies needed for the specified accident, and Constraint (3) ensures the need of a certain type of vehicles requested to the accident. Constraint (4) ensures that there always must be at least one person assigned to a vehicle if it should be possible to use the vehicle. Since a vehicle only has a specified number of seats available, only a maximum number of persons can be assigned to each specific vehicle. Constraint (5) therefore ensures that the vehicle seating capacity is not exceeded, and also that a driver is assigned to each responding vehicle. Then, 
if a person should be able to contribute to the preparedness for the accident, it must be a vehicle available to make sure that the person can go to the accident site. That condition is ensured by Constraint (6).

Furthermore, a person can be assigned to only one vehicle, only one of a person's competencies can be utilized at a time, and a person can only contribute with a certain competency if the person possesses the competency. These conditions and restrictions are ensured by Constraints (7), (8) and (9). Constraint (10) guarantees that a person only can be assigned to a vehicle if the person is available and permitted to be assigned to that vehicle. A person can for example only be assigned to a vehicle if they are located at the same place. Also, a vehicle can only be driven by one person at a time, and a person can only drive one vehicle at a time. This is ensured by Constraints (11) and (12) respectively. The person who drives a vehicle must also always be assigned to that vehicle, i.e. the person travels in that vehicle to the accident site. That condition is ensured by Constraint (13). Since persons sometimes must have specific qualities or skills to be able to drive certain vehicles, Constraint (14) is included in the model. This condition ensures that a person only can drive a certain vehicle if the person is permitted and allowed to drive the vehicle.

Constraint (15) and (16) represent the response time for the vehicles and the persons that are contributing to the preparedness, i.e. the selected resources. The response time for a vehicle is the vehicle's travel time plus the preparation time for the person assigned to the vehicle with the longest preparation time. The response time for a person is the travel time for the vehicle, which the person is assigned to, plus the preparation time for the person assigned to the same vehicle that has the longest preparation time. Constraint (17) confirms binary variables and Constraint (18) ensures non negativity.

\subsection{Solution Method for the Resource Selection Model}

Solutions to the resource selection model have been produced by the mathematical programming solver Gurobi Optimizer 5.6.0 for different areas and different scenarios. For the different scenarios, the demand for resources, the number of demand zones and the available resources have been varied. When the resource selection model has been solved optimally by Gurobi Optimizer 5.6.0 for various problems, the solution time has proven to be too long. The solution time for the produced results has been somewhere around 1.5 to 8 seconds when using a PC laptop, with an Intel Core i5-2520M, $2.50 \mathrm{GHz}$ processor, and $8 \mathrm{~Gb}$ RAM. To solve the model for approximately 2500 demand zones, will thus take around 1 to 5.5 hours. 2500 demand zones are not unusual for these types of problems. The solution time is consequently unacceptably long for the model to be useful.

To reduce the solution time for solving the resource selection model, a heuristic using a greedy based algorithm is developed.

\subsubsection{The Heuristic Developed for Solving the Resource Selection Model}

The developed heuristic for solving the resource selection model consists of a greedy based search procedure that search for the specific requested resources from the present available 
ones. The heuristic searches for the closest available resources that are requested since the objective is to minimize the response time.

In the search procedure, lists are created for both the requested and the available resources. Lists are created separately for vehicles and firefighters, and are created in order to find the requested resources from the available resources. For example, the algorithm searches the requested firefighters from the available firefighters.

The requested firefighters are stored in a list $L_{\text {Firefighters }}^{r}$. First, the list of the requested firefighters is sorted depending on the competency $r$ that is requested. The scarcest competency is found at the top of the list, and the most common in the bottom of the list. The scarcest competency is the one that is most difficult to find among the firefighters. That means the algorithm will always start to search for the scarcest requested competencies. The requested vehicles do not need to be sorted in any particular order, since a vehicle can only be of a certain type. The requested vehicles are stored in a list $L_{V e h i c l e s}$. A summary of the used lists for the requested resources are found in Table 10.

Table 10. The used lists for the requested resources

Lists for requested resources Sort order

List of the requested firefighters, $L_{\text {FireFighters }}^{r}$

Sorted based on the requested competency $r$. The scarcest competency is found at the top of the list and the most common competency in the bottom of the list.

(Only one competency can be requested at a time)

List of the requested vehicles, $L_{V \text { ehicles }}$

No sort order is needed

The lists of available resources, both firefighters and vehicles, are sorted in ascending order according to their expected response times. This means, the available resources with the quickest response time are found at the top of the list. However, if several available firefighters have the same response time, e.g. if they are located at the same fire station, they are then also sorted depending on $c$, the number of competencies $r$ each firefighter possesses (since they can have multiple competencies). If also the number of competencies is the same for several firefighters, they are sorted in availability order based on which main competencies $r$ the firefighters have. The firefighters are sorted in a way so that the firefighters with the most common competencies will be selected before firefighters with scarcer competencies. The available firefighters are sorted in a list $L_{\text {Firefighters }}^{\text {tcr }}$ The list of available firefighters are thus first sorted in ascending order according to their response time $t$, then in ascending order according to $c$, their number of competencies, and then in availability order according to the main competency $r$. The firefighters with the fewest and the most common competencies are thus found at the top of the list. Firefighters with scarce and many competencies are found in the bottom of the list. The available vehicles are stored in a list $L_{\text {Vehicles }}^{t}$, and the vehicles with the quickest response time will be found at the top of the list. A summary of the lists used for the available resources are presented in Table 11. 
The reason why the firefighters are sorted in this way is to prevent that a firefighter with multiple and scarce competencies is selected to assignments it might be over qualified for. For example, assume that the requested firefighters consist of one BA (Breathing Apparatus) team leader and one ladder vehicle operator and the nearest available resources is one firefighter with BA rescue competency and one firefighter with both the BA rescue competency and ladder vehicle competency. In this case, it is obviously better to select the firefighter with the $\mathrm{BA}$ rescue competency to be selected as the BA team leader and the firefighter with both the competencies to be selected as the ladder vehicle operator. If the firefighter with both the competencies should have been selected as the BA team leader, it should be necessary to select the requested ladder vehicle operator from another place, probably a place further away. To avoid the later situations, the firefighters are sorted in specific orders in the heuristic.

Table 11. The used lists for the available resources

\begin{tabular}{ll}
\hline Lists for available resources & Sort order \\
\hline \hline List of the available firefighters, $L_{\text {Firefighters }}^{\text {tcr }}$ & $\begin{array}{l}\text { Sorted in ascending order according to the firefighters } \\
\text { response time } t \text {, then in ascending order according to the } \\
\text { firefighters number of competencies } c \text {, and then in } \\
\text { availability order according to the firefighters main } \\
\text { competency } r .\end{array}$ \\
List of the available vehicles, $L_{\text {Vehicles }}^{t}$ & $\begin{array}{l}\text { Sorted in ascending order according to the vehicles response } \\
\text { time } t .\end{array}$ \\
\hline
\end{tabular}

Finally, the algorithm searches for the requested firefighters in the list $L_{\text {FireFighters }}^{r}$, and starts from the top of the list. When finding the first matching available firefighter in the list $L_{\text {FireFighters }}^{t c r}$, it will be selected. The search continues until all the requested firefighters in the list $L_{\text {Firefighters }}^{r}$ have been found. Then, the algorithm searches for the requested vehicles in the list $L_{\text {Vehicles. }}$. When finding the first matching available vehicle in the list $L_{\text {Vehicles }}^{t}$, it will be selected. However, there must always be available vehicles that can transport the firefighters and available firefighters that can drive the vehicles; otherwise the resources cannot be selected. The search process will in that case continue and the next element in the list of the available resources will be checked.

The pseudo code for the heuristic used to solve the resource selection model is presented in Table 12 . 
Table 12. Pseudo code for the heuristic developed for solving the resource selection model

The pseudo code for the heuristic for solving the resource selection model

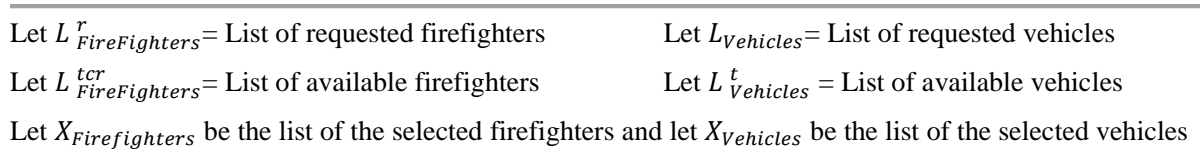

Foreach ( requested firefighter $r f$ in $L_{\text {FireFighters }}^{r}$ )

Foreach (available firefighter af in $L_{\text {FireFighters }}^{\text {tcr }}$

If ( $a f=r f \quad \& \&$ there is an accessible vehicle(and a driver) that can transport the available firefighter af from its current location to the intended destination)

$$
\begin{aligned}
& X_{\text {Firefighters }} \cdot \operatorname{Add}(a f) \\
& L_{\text {FireFighters }}^{\text {tcr }} . \operatorname{Remove}(a f)
\end{aligned}
$$

Else

No matching available firefighter can be found

Foreach (requested vehicle $r v$ in $L_{\text {Vehicles }}$ )

Foreach (available vehicle $a v$ in $L_{\text {Vehicles }}^{t}$ )

If $(a v=r v \& \&$ there is an accessible person able to drive the available vehicle $a v$ from its current location to the intended destination)

$$
\begin{aligned}
& X_{\text {Vehicles }} \cdot \operatorname{Add}(a v) \\
& L_{\text {Vehicles }}^{t} \cdot \operatorname{Remove}(a v)
\end{aligned}
$$

Else

No matching available vehicle can be found

If (All the requested resources are found)

Return $X_{\text {Firefighters }}$ and $X_{\text {Vehicles }}$

$\rightarrow$ The preparedness can be measured for the certain situation.

Else

\section{Return null}

There is no solution to the current resource selection problem.

$\rightarrow$ The preparedness cannot be measured for the certain situation.

\subsection{Validation of the Developed Heuristic}

To validate the developed heuristic, i.e. investigate if the heuristic is adequate to use for solving the resource selection problem in order to measure the preparedness for different situations, solutions produced by the heuristic has been compared to solutions produced by the mathematical programming solver Gurobi Optimizer 5.6.0. In order to validate the heuristic, the resource selection model has been solved both by Gurobi and heuristically for 18 different scenarios in a test setting.

\subsubsection{The Test Setting}

The test setting, used in order to validate the heuristic, consists of a geographical area divided into 2584 zones, á 733x733 meters. The area is based on a region in Denmark, which has been somewhat altered to better fit the purpose of the tests (e.g. some population were removed 
from border areas to avoid border issues and some vegetation were changed). Travel times between the zones in the area are based on Open Street Map data (http://www.openstreetmap.org/) and were generated using Network Analyst in ArcMap 10. The forecast of expected number of accidents per year in each zone for each accident type has been based on values for corresponding similar areas, and has been validated as reasonable by experts from the fire and rescue service. More information on how the test setting was constructed can be found in Lundberg et al. (2012).

A total of 43 vehicles of seven different types are included in the test setting. Furthermore, the setting includes 61 firefighters (persons included in the model), which can have one or more of seven personnel competencies. Most firefighters have more than one competency, and they can have up to four. A firefighter can however only manage and be utilized for one competency at a time (but, if a firefighter is the first responding person (FiP) to an accident, it can also assist with its regular competency as well). It is thus only one competency that can be requested at a time. The personal competencies available in the test setting are incident commanders (IC), external commanders (EC), team leaders (TL), BA team leader (BA), ladder vehicle operators (LO), basic firefighters (BF) and first responders (FiP). That firefighters may have multiple competencies is the major factor that contributes to the problem complexity of assigning firefighters to an accident. The different types of firefighters, their competencies and the number of firefighters that are included in the test setting are presented in Table 13.

Table 13. The firefighters included in the test setting

\begin{tabular}{llll}
\hline Firefighters included in the test setting & The competencies $\boldsymbol{r}$ the \\
and their main competency & firefighter possesses & $\begin{array}{l}\text { Number included in } \\
\text { the test setting }\end{array}$ \\
\hline \hline 1 & Incident Commander (IC) & (IC) (EC) (FiP) & 1 \\
2 & External Commander (EC) & (EC) (TL) (FiP) & 3 \\
3 & Team Leader (TL) & (TL) (BA) (BF) (FiP) & 9 \\
4 & BA Team Leader \& Ladder vehicle Operator & (BA) (LO) (BF) (FiP) & 3 \\
& (BA \& LO) & (LO) (BF) (FiP & 6 \\
6 & Ladder vehicle Operator (LO) & (BA) (BF) (FiP) & 15 \\
5 & BA Team Leader (BA) & (BF) (FiP) & 14 \\
7 & Basic Firefighter (BF) & (FiP) \\
\hline
\end{tabular}

According to Table 13 there are 47 persons that can respond as a basic firefighter (BF), 18 that can respond as a BA team leader (BA), 12 that can respond as a team leader (TL), 9 that can respond as a ladder vehicle operator (LO), 4 that can respond as an external commander (EC) and one that can respond as an incident commander (OC). In Table 14, the personal competencies $(r)$ included in the test setting are listed in ascending order according to their availability. The most unusual competencies among the fire service staff are found at the top 
of the table and the most common in the bottom. Notice that this list varies depending on the resources that are included.

Table 14. Sort order for the personal competencies $r$ that are included in the test setting

\begin{tabular}{ll}
\hline Sort order & Personal Competency \\
\hline \hline & \\
2 & Incident Commander (IC) \\
3 & External Commander (EC) \\
4 & Ladder vehicle Operator (LO) \\
5 & Team Leader (TL) \\
6 & BA Team Leader (BA) \\
7 & Basic Firefighter (BF) \\
& First Responder (FiP) \\
\hline
\end{tabular}

The different types of vehicles and the number of different types of vehicles that are included in the test setting are presented in Table 15. To drive or manage certain vehicles sometimes requires a specific competency or competencies; e.g. to drive a ladder vehicle one ladder vehicle operator is required, and two ladder vehicle operators are needed to use the ladder vehicle at an accident site. Furthermore, first responders (FiP) can only drive first response vehicles (FiV) and ladder vehicles can only be driven by ladder vehicle operators. Also, command vehicles (CV) can only be driven by external commanders (EC) and incident commanders (OC). All firefighters can be transported as passengers in any vehicle, and all the additional vehicles (except ladder vehicles and command vehicles) can be driven by any personnel resource.

Table 15. The vehicles included in the test setting

\begin{tabular}{|c|c|c|c|}
\hline & Vehicles included in the test setting & $\begin{array}{l}\text { Type of vehicle (number of seats in the } \\
\text { vehicle ) }\end{array}$ & $\begin{array}{l}\text { Number included } \\
\text { in the test setting }\end{array}$ \\
\hline 1 & Fire Vehicle (FV) & Vehicle with base equipment. (5) & 10 \\
\hline 2 & Ladder Vehicle (LV) & $\begin{array}{l}\text { Vehicle with access to a } 30 \text {-meter ladder. } \\
\text { (2) }\end{array}$ & 3 \\
\hline 3 & First Response Vehicle (FiV) & Vehicle with first response equipment. (4) & 19 \\
\hline 4 & Command Vehicle (CV) & $\begin{array}{l}\text { Fire officer's vehicle with first response } \\
\text { equipment. (2) }\end{array}$ & 3 \\
\hline 6 & Tanker (TV) & $\begin{array}{l}\text { Vehicle with extra-large water supply, } \\
\text { complement to the fire vehicle. (2) }\end{array}$ & 5 \\
\hline 5 & Special Vehicle (SV) & $\begin{array}{l}\text { Vehicle with special equipment for } \\
\text { different types of extraordinary } \\
\text { emergencies. ( } 2 \text { ) }\end{array}$ & 2 \\
\hline 7 & Special Chem Vehicle (ChV) & $\begin{array}{l}\text { Vehicle with special equipment for } \\
\text { handling chemicals and hazardous } \\
\text { emissions. ( } 2 \text { ) }\end{array}$ & 1 \\
\hline
\end{tabular}


The resource selection model has been solved for three different types of accidents - traffic accidents, building fire accidents and high-rise building fire accidents. The required resources for each type of accident are presented in Table 16. Even if not all the types of resources are required, the additional personnel resources might be used to sometimes drive required vehicles, and the additional vehicles might be used to transport required personnel resources.

Table 16. Resource requirements for the different types of accidents.

\begin{tabular}{lll}
\hline Type of Accident & Requested personnel & Requested vehicles \\
\hline \hline \multirow{2}{*}{ Traffic Accidents } & $1 \times$ Team Leader & 1 x Fire Vehicle \\
& $4 \times$ Basic Firefighters & \\
Building Fires & $1 \times$ Team Leader & $1 \times$ Fire Vehicle \\
& $1 \times$ BA Team Leader & \\
& $3 \times$ Basic Firefighters & \\
& & $1 \times$ Fire Vehicle \\
Building Fires in High-Rise Buildings & $1 \times$ Team Leader & $1 \times$ Ladder Vehicle \\
& $1 \times$ BA Team Leader & \\
& $2 \times$ Ladder Vehicle Operators & \\
& $3 \times$ Basic Firefighters & \\
\hline
\end{tabular}

The test setting consists of 2584 demand zones. However, all the zones do not contain expected number of accidents for all three accident types. There are 2282 zones with expected building fire accidents, 2375 with expected traffic accidents and 99 zones with expected highrise building fire accidents. To produce solutions for a scenario in the test setting, in order to measure the preparedness for the three types of accidents, the resource selection model must be solved for all zones with expected demand and also for each type of accident. This means there are 4756 resource selection problems that must be solved for each scenario.

\subsubsection{Solutions of the Test Scenarios}

The resource selection problem has been solved for 18 different scenarios in the test setting explained in the previous section. The things that differ between the scenarios are the availability of resources and the location of the available resources. That means, all the 43 vehicles and the 61 firefighters included in the test setting are not available in all various scenarios. The numbers of vehicles that are available vary between 25 and 43 in the scenarios, and the numbers of firefighters that are available vary between 24 and 61 . All the resources are only available in one scenario.

Results have been obtained for all 18 scenarios by using a PC laptop, with an Intel Core i52520M, 2.50 GHz processor, and 8 Gb RAM. The solution time for solving the resource selection model for one scenario, i.e. 4756 times, using Gurobi varied between 2 and 10 hours. The corresponding solution time for the heuristic varied between 7 and 10 seconds for the different scenarios. When the contributing resources were determined for each type of accident, for each scenario, the preparedness was measured. Results are presented in Table 17. 


\subsubsection{Computational Results}

Table 17 presents aggregated results for each scenario, where optimal and heuristic solutions are found for all accident types and zones. In most cases the heuristic finds an optimal solution, as indicated by the fourth column in Table 17, which displays the number of times the heuristic fails. In 11 of the 18 scenarios, the heuristic always finds the optimal solution. In Scenario 2 and 12, it delivers inferior solutions in 37-50\% of the problems solved. However, looking at the difference in objective function values, it is only in Scenario 2 that the solutions produced by the heuristic are noticeably worse than the optimal solution. The reason for this is probably that the resources in Scenario 2 are located in a manner that makes it difficult for the heuristic to perform optimal selections. For the other scenarios, the mean value of the preparedness (measured over all zones and accident types) differs less than one percent. Also, since it is the same resource selection problem that is solved for all zones, the same resources are probably selected for many zones in the same surrounding area, i.e. the heuristic make the same selection (error) many times.

Table 17. Computational results comparing the heuristic solutions to the optimal solutions

\begin{tabular}{|c|c|c|c|c|c|c|}
\hline Scenario & $\begin{array}{l}\text { Number of } \\
\text { available } \\
\text { firefighters } \\
\text { (Excluding } \\
\text { FiP) } \\
\end{array}$ & $\begin{array}{l}\text { Number of } \\
\text { available } \\
\text { vehicles } \\
\text { (UB \& UL) }\end{array}$ & $\begin{array}{l}\text { Number of } \\
\text { problems where } \\
\text { the objective } \\
\text { function values } \\
\text { differs }\end{array}$ & $\begin{array}{l}\text { Mean } \\
\text { preparedness } \\
\text { for optimal } \\
\text { solutions }\end{array}$ & $\begin{array}{l}\text { Mean } \\
\text { preparedness } \\
\text { for heuristic } \\
\text { solutions }\end{array}$ & $\begin{array}{l}\text { Difference } \\
\text { [\%] }\end{array}$ \\
\hline 1 & 50 & 13 & 0 & 7.610 & 7.610 & 0 \\
\hline 2 & 14 & 6 & 2383 & 12.610 & 13.435 & 6.5 \\
\hline 3 & 26 & 7 & 33 & 10.369 & 10.385 & 0.1 \\
\hline 4 & 20 & 8 & 0 & 9.483 & 9.483 & 0 \\
\hline 5 & 23 & 8 & 18 & 9.085 & 9.092 & 0.1 \\
\hline 6 & 34 & 9 & 4 & 8.557 & 8.557 & 0 \\
\hline 7 & 33 & 9 & 86 & 8.717 & 8.722 & 0.1 \\
\hline 8 & 14 & 5 & 0 & 10.719 & 10.719 & 0 \\
\hline 9 & 18 & 5 & 0 & 11.540 & 11.540 & 0 \\
\hline 10 & 25 & 8 & 0 & 9.458 & 9.458 & 0 \\
\hline 11 & 28 & 11 & 0 & 8.786 & 8.786 & 0 \\
\hline 12 & 25 & 8 & 1769 & 10.509 & 10.547 & 0.4 \\
\hline 13 & 37 & 11 & 0 & 7.987 & 7.987 & 0 \\
\hline 14 & 24 & 10 & 0 & 9.173 & 9.173 & 0 \\
\hline 15 & 32 & 9 & 91 & 8.794 & 8.796 & 0.0 \\
\hline 16 & 27 & 8 & 0 & 9.530 & 9.530 & 0 \\
\hline 17 & 29 & 9 & 0 & 9.814 & 9.814 & 0 \\
\hline 18 & 23 & 7 & 0 & 10.878 & 10.878 & 0 \\
\hline
\end{tabular}


Furthermore, from the second and the third columns in Table 17 we can see that the number of available resources does not affect the result in any particular meaning. It is probably the resources locations and how they are combined in the area that affect how they are selected in the heuristic compared to how they are selected in the optimal solutions. In the second and in the third columns, it is only the number of resources that can contribute to the preparedness that are included. Also, when the heuristic solutions differ from the optimal, we have seen that it is always the selection of firefighters that differ. The vehicles are always selected in the same way, both in the heuristic and by Gurobi. This is probably due to that the firefighters may have multiple competencies, which also is the reason to the problem complexity.

Calculating means over a large number of zones might dilute the results, so therefore, Table 18 presents only results for the problems where the heuristic does not find the optimal solution.

In Table 18 results are aggregated over zones, but presented separately for the three accident types; Traffic Accidents (TA), Building Fires (BF) and Building Fires in High-rise Buildings $(\mathrm{HBF})$. For Scenario 2, the objective functions for the heuristic solutions have a mean value $11 \%$ higher than the optimal, both when considering building fires and traffic accidents, and slightly higher for the high-rise building fires. Comparing results for the different accident types must be done carefully since each column is an aggregation of different problems sets, e.g. each scenario for HBF contains 99 problems, while BF and TA have over 2000 problems each. With this in mind, HBF seem to offer more of a challenge when selecting resources, as the largest mean difference between optimal and heuristic objective functions values is found in Scenario 5 for high-rise building fires (21.7\%). This is however hardly surprising, as more resources are required for fires in high-rise buildings than for regular building fires or traffic accidents. It also makes sense that the resource selection problem gets more complicated when more resources are required (up to a certain point when you just have to send everything that you have got). This is somewhat contradicted by the fact the maximum difference (31.9\%) between the heuristic and the optimal solutions for any single solution is found for a traffic accident, which has the simplest resource requirement of the three accident types. However, some zones have expected number of traffic accidents, but no expected building fires (e.g. in zones where there are roads but no buildings). It is also possible that zones only have expected building fires, but no traffic accidents. Depending on the resources that are selected, it is the response times that determine the difference between the heuristic and optimal solutions. The zone where the maximum difference is found is a zone that only contains expected traffic accidents and where the closest resources have quite long response times. When only studying zones where both fire and traffic accidents can occur, the heuristic - just as the exact solver - always finds solutions with equal or shorter mean response times for traffic accidents than for fire accidents. This is because the same resources are required for both accident types, but one additional competency (BA operator) is needed for fire accidents. 
Table 18. Results for the zones where the preparedness differ depending on the solution method

\begin{tabular}{lllllll}
\hline Scenario & $\begin{array}{l}\text { Mean } \\
\text { difference } \\
\text { for BF [\%] }\end{array}$ & $\begin{array}{l}\text { Max } \\
\text { difference } \\
\text { for BF [\%] }\end{array}$ & $\begin{array}{l}\text { Mean } \\
\text { difference } \\
\text { for TA [\%] }\end{array}$ & $\begin{array}{l}\text { Max } \\
\text { difference } \\
\text { for TA [\%] }\end{array}$ & $\begin{array}{l}\text { Mean } \\
\text { difference } \\
\text { for HBF [\%] }\end{array}$ & $\begin{array}{l}\text { Max } \\
\text { difference } \\
\text { for HBF [\%] }\end{array}$ \\
\hline \hline 2 & 10.9 & 27.9 & 11.5 & 31.9 & 12.9 & 15.9 \\
3 & & & & & 17.5 & 23.4 \\
5 & & & & & 21.7 & 30.7 \\
6 & 0.6 & 1.2 & & & 1.2 & 1.6 \\
7 & 3.5 & 6.8 & 0.8 & 12.0 & 2.9 & 4.5 \\
15 & 0.7 & 7.0 & 2.2 & 0.7 & 0.9 & \\
\hline
\end{tabular}

In total, 85608 problems were solved heuristically and exactly. In $95 \%$ of the tested cases, the heuristic did find an optimal solution. In the cases where the heuristic did not find an optimal solution, the heuristic solutions were never more than $32 \%$ from the optimal; see Table 18 . Thus, the conclusion is that the heuristic is good enough to be used in order to measure the preparedness, at least for the specific test setting used here. No major deviations in the solution methods are indicated in the results.

However, it should be noted that even if the heuristic most often finds an optimal solution, it is not necessarily the same solution that is found by the exact solver. Several different resources (firefighters) can contribute with the same competency, and therefore there are several resources that can be selected for the same competency. Thus, a problem often has multiple optimal solutions.

\subsection{Calibration and Validation of the Preparedness Value}

To be able to adequately utilize the preparedness measures, for example as shown in Section 5.1.1, the preparedness measure has to be calibrated and validated. In this section, a procedure to calibrate and validate the preparedness measure for the test area described in Section 5.4.1 is proposed.

The preparedness measure has been calibrated and validated for the test setting using preparedness evaluations made by professional staff from different fire and rescue service in Sweden. The evaluated results (the perceived preparedness) have been compared against the measured results from a number of scenarios.

\subsubsection{The Evaluation Process}

In the evaluation process, the 18 scenarios plus an additional six scenarios have been evaluated by fire and rescue service personnel. The process consisted of evaluating the preparedness. For each scenario, the preparedness has been graded for 13 selected zones of the test setting. An example is shown in Figure 11, where seven of the 13 zones is shown and 
marked as A - G. The test setting includes a total of nine fire stations. In Figure 11, three of these are shown, and numbered 20, 26 and 24. Number 26 and 24 are part-time fire stations and number 20 is a full time fire station. In each scenario of the test setting, there are also a number of available resources consisting of different firefighters and vehicles. As mentioned before, the type and number of available resources - as well as their location - are different in each scenario (the resources are therefore not included in the figure).

The assignment for the fire and rescue service personnel was to evaluate the preparedness in each of the 13 zones, taking into account which resources that are available, and where these are located. The experts were provided with maps visualizing the number of expected accidents as well as expected travel times in the area. The fire and rescue service personnel that evaluated the preparedness consisted of incident commanders.

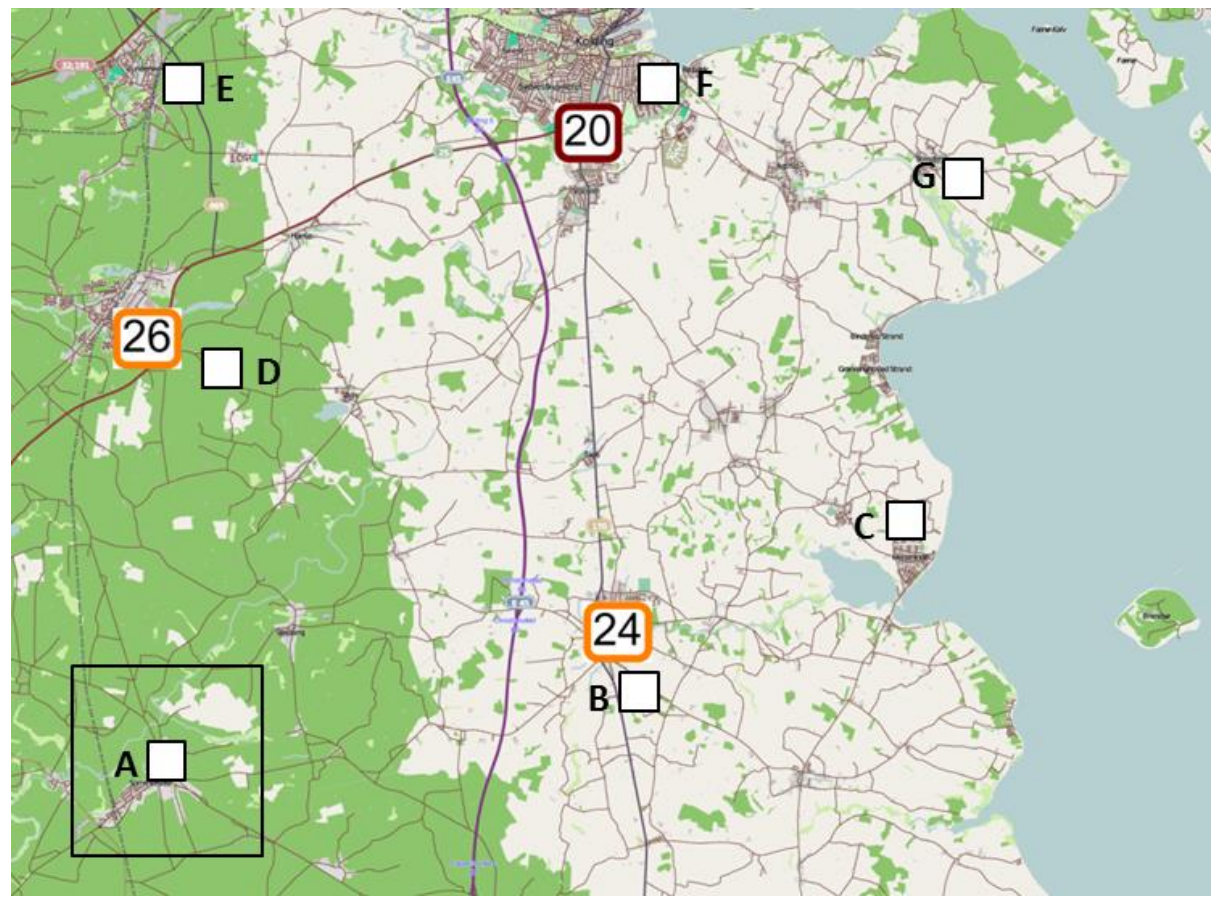

Figure 11. Area of zones included in the evaluation process

For all 13 zones, the experts from the fire and rescue service were instructed to grade the preparedness as good, acceptable or inadequate, taking into account the specific zone, as well as the area surrounding the specific zone. Furthermore, the experts evaluated the preparedness for all accident types (traffic, building fires and building fires in high-rise buildings) together. Thus, all the accident types were considered simultaneously in the evaluations. When the preparedness was measured, separate measurements were made for each of the three accident types. To be able to compare the evaluated preparedness with the measured, the worst (highest) measured preparedness for the three accidents types was assumed to correspond to the overall preparedness evaluated by the fire and rescue service staff. The worst preparedness was considered because an expert was assumed not to consider the preparedness as adequate 
or good if it was inadequate for any of the accident types. Thus, the max measured preparedness $P_{j}^{\max }$ for each zone $j$ was used in the process of comparing the measured preparedness with the evaluated preparedness, i.e. the measured value:

$$
P_{j}^{\max }=\max _{a \in A} P_{a j} \quad \forall j \in J
$$

$P_{a j}$ is the measured preparedness for accident of type $a$ in zone $j$. Furthermore, the preparedness was evaluated for the zones, including their surrounding areas (see an example in Figure 11, around Zone A). But when the preparedness is measured, it is measured for each zone. Therefore, the preparedness measure for the center zone, along with the preparedness measures for two layers of neighboring zones, were included when the evaluated preparedness was compared with the measured. For each layer, the weight of the contribution was also assumed to decrease. The center zone got $50 \%$ of the weight, the first layer $30 \%$ and the second layer $20 \%$. For each zone $j$ in the test setting, the preparedness value that was compared to the expert evaluations was calculated as:

$$
P_{j}^{\text {Comp }}=0.5 P_{j}^{\max }+0.3\left(\frac{\sum p_{k}^{\text {max }}}{8}\right)+0.2\left(\frac{\sum p_{l}^{\max }}{16}\right) \quad \forall j \in J
$$

where the sum over index $k$ is of all max preparedness values in the first layer of neighboring zones around $j$, and the sum over index $l$ is of all max preparedness values in the second layer of neighboring zones around $j$.

\subsubsection{A Model for Finding Thresholds}

One purpose of the calibration process was to find thresholds for the preparedness measure, so that it is possible to say if the measured values correspond to good or inadequate preparedness. To find such thresholds, an integer programming model with the objective of minimizing the deviation between the measured preparedness and the perceived preparedness was constructed and solved.

The objective of the model is to find thresholds $R$ and $S$, so that the number of evaluations where the preparedness as perceived by the fire and rescue service personnel differs from the measured value is minimized. A measured preparedness value above $R$ indicates that the preparedness is inadequate (and would be visualized as red in Figure 9), while a value below $S$ means that the preparedness is good. Values between $R$ and $S$ correspond to acceptable preparedness.

The evaluations made by the fire and rescue service personnel were gathered into three sets, I, $A$ and $G$, where each evaluation corresponds to one assessment of one situation in one zone by one person. Depending on the assessment, the evaluation will end up in one of the sets; e.g. if the expert deemed the preparedness as acceptable, the evaluation belongs to set $\mathrm{A}$.

\section{Sets:}

$I=$ the set of evaluations where the preparedness was considered inadequate

$A=$ the set of evaluations where the preparedness was considered acceptable

$G=$ the set of evaluations where the preparedness was considered good 


\section{Variables:}

$R=$ threshold for when the preparedness is considered inadequate (if exceeded)

$S=$ threshold for when the preparedness is considered good (if below)

$x_{i}^{I R}=\left\{\begin{array}{l}1, \text { if evalution } i \text { has a measured value below the threshold } R \\ 0, \text { otherwise }\end{array}\right.$

$x_{i}^{I S}=\left\{\begin{array}{l}1, \text { if evalution } i \text { has a measured value below the threshold } S \\ 0, \text { otherwise }\end{array}\right.$

$x_{j}^{A S}=\left\{\begin{array}{l}1, \text { if evalution } j \text { has a measured value below the threshold } S \\ 0, \text { otherwise }\end{array}\right.$

$x_{j}^{R A}=\left\{\begin{array}{l}1, \text { if evalution } j \text { has a measured value above the threshold } R \\ 0, \text { otherwise }\end{array}\right.$

$x_{k}^{S G}=\left\{\begin{array}{l}1, \text { if evalution } \mathrm{k} \text { has a measured value above the threshold } S \\ 0, \text { otherwise }\end{array}\right.$

$x_{k}^{R G}=\left\{\begin{array}{l}1, \text { if evalution } k \text { has a measured value above the threshold } R \\ 0, \text { otherwise }\end{array}\right.$

\section{Parameters:}

$r_{i}=$ measured preparedness value for evaluation $i \in I$

$s_{j}=$ measured preparedness value for evaluation $j \in A$

$t_{k}=$ measured preparedness value for evaluation $k \in G$

$M=$ a large number

Minimize $\sum_{i \in I}\left(x_{i}^{I R}+x_{i}^{I S}\right)+\sum_{j \in A}\left(x_{j}^{A S}+x_{j}^{R A}\right)+\sum_{k \in G}\left(x_{k}^{S G}+x_{k}^{R G}\right)$

\section{Subject to}

$R-r_{i} \leq M x_{i}^{I R}$

$\forall i \in I$

$S-r_{i} \leq M x_{i}^{I S}$

$\forall i \in I$

$S-s_{i} \leq M x_{j}^{A S}$

$\forall j \in A$

$s_{i}-R \leq M x_{j}^{R A}$

$\forall j \in A$

$t_{k}-S \leq M x_{k}^{S G}$

$\forall k \in G$

$t_{k}-R \leq M x_{k}^{R G}$

$\forall k \in G$ 
$x_{i}^{I R}, x_{i}^{I S}, x_{j}^{A S}, x_{j}^{R A}, x_{k}^{S G}, x_{k}^{R G} \in\{0,1\}$

$R, S \geq 0$

For each preparedness evaluation, the developed preparedness measure is also used to calculate the preparedness, which gives the parameters $r, s$ and $t$. The $x$-variables take the value of 1 each time that there is a mismatch between a measured value and a perceived value. For example, in constraint (2), if the value for $r_{i}$ is below the threshold $R$, the variable $x_{i}^{I R}$ is forced to take the value of 1 due to the mismatch. Lowering $R$ to compensate for this mismatch might cause problems in, e.g. constraint (5) where the measured preparedness for an evaluation, where the preparedness is considered acceptable, then may end up above the threshold $R$, which is not desired. Each evaluation is compared to the two thresholds. The objective function (1) is the sum of the $x$-variables, and the objective function value is, thus, the number of times where the perceived and the measured preparedness values do not correspond, plus the extra penalty for the occasions when the measured preparedness value is on the wrong side of both thresholds. For example, if $x_{k}^{R G}$ is one, then $x_{k}^{S G}$ must also be one, since the measured preparedness value $t_{k}$ cannot be higher than the value of $R$ (the threshold value for inadequate preparedness) without being higher than value of $S$ (the threshold value for good preparedness).

\subsubsection{Results from Calibration}

Twelve experts have contributed to the evaluation of the 24 scenarios. Each scenario was evaluated by two experts, and with 13 zones in each scenarios, this gives a total of 623 evaluations. Of this total, 208 were perceived as inadequate, 208 as acceptable and 207 as good. This in itself is an interesting result.

For example, the scenarios consist of various types and numbers of available resources, some with many and some with fewer available resources, which means that the preparedness is evaluated for different states. Given that the evaluations are subjective and performed by multiple individuals, it is unexpected that the evaluations would be evenly distributed. 


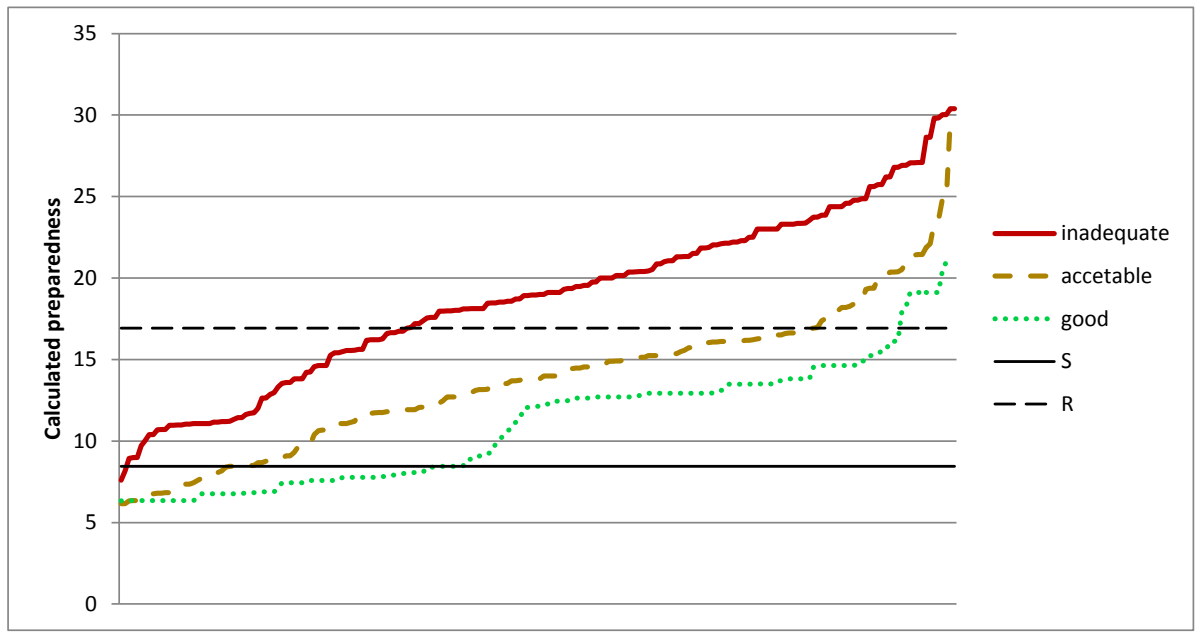

Figure 12. Results from comparing measured preparedness with perceived preparedness

The results from the evaluations compared to the measured preparedness values are presented in Figure 12. Each set $(I, A, G)$ is represented by a line where the measured values of the evaluations have been sorted in increasing order. The dotted line shows, for example, the elements in the set $G$ (the evaluations where the preparedness is considered good). The vertical axes, then, represent the corresponding measured preparedness values. As can be seen in the figure, there is a significant overlap between the sets, making it impossible to identify thresholds that do not give any mismatch between perceived and measured preparedness.

The IP model was solved using Frontline Systems' Analytic Solver Platform in MS Excel. An optimal solution was produced in less than a minute with an objective function value of 271 . The optimal variable values are presented in Table 19.

Table 19. Variable values from the calibration run

\begin{tabular}{lcccccccc}
\hline Variable & $S$ & $R$ & $x_{i}^{I R}$ & $x_{i}^{I S}$ & $x_{j}^{A S}$ & $x_{j}^{R A}$ & $x_{k}^{S G}$ & $x_{k}^{R G}$ \\
\hline Value & 8.45 & 16.93 & 71 & 2 & 27 & 35 & 123 & 13 \\
\hline
\end{tabular}

The optimal thresholds give a result where there is a match between the perceived and the measured preparedness in 367 occasions out of the 623 (the extra penalties given by the variables $x_{i}^{I S}$ and $x_{k}^{R G}$ are not included in this calculation). One interpretation of this is that there is a $60 \%$ chance that an expert from the fire and rescue service will agree with the measured preparedness, if the optimal thresholds are used. Of course, it would be desirable to have a higher matching rate, but unfortunately, the fire and rescue service personnel are not consistent with each other in their evaluations, even when judging the same scenario (this result also matches the one reported in Andersson Granberg, 2013). 


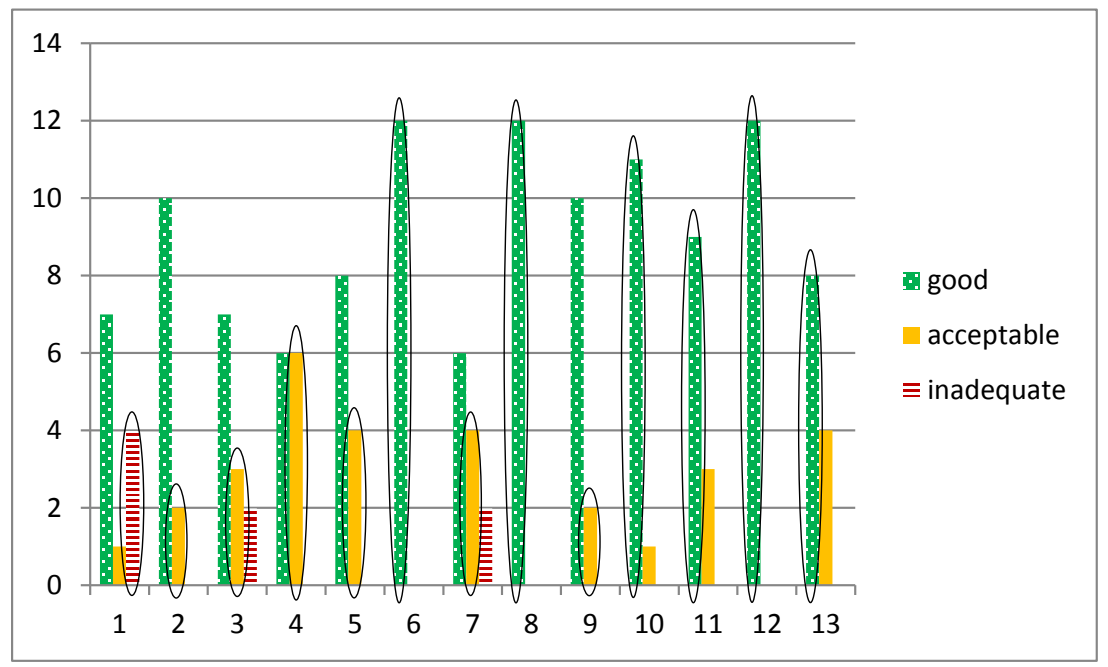

Figure 13. The results from 12 experts' perceived preparedness for one scenario

Figure 13 shows for example that the fire and rescue service personnel sometimes are inconsistent when evaluating the preparedness. The figure shows the results where 12 experts have judged the preparedness for the same scenario in the 13 zones. The particular scenario is one where all resources are available and positioned at their respective fire stations, which means that the scenario should be fairly easy to evaluate. The measured preparedness levels are indicated by circling the corresponding column in Figure 13. In three (zone 6, 8 and 10) of the 13 zones, all 12 experts, as well as the developed measure, agree that the preparedness is good. In the rest of the zones, there are varying levels of disagreement, which are especially noteworthy in zone 1, 3 and 7, where some experts deem the preparedness to be good, while other experts consider it to be inadequate. In this particular scenario, the measure tends to calculate the preparedness as slightly worse than a majority of the experts; see zone 1, 2, 3, 5, 7 and 9. This was partly explained by comments from some of the experts during the evaluations, that "if all resources are available at the station, the preparedness must be good".

As previously mentioned, the scenario evaluated in Figure 13, is one of the less complex scenarios that the experts had to assess. When the complexity increases, so does the variation in the evaluations. The use of a preparedness measure might help to decrease this variation, and create a common understanding for the preparedness concept. A $60 \%$ hit rate between the measured and the perceived preparedness was therefore considered good enough by the authors.

\subsection{Conclusions}

In this chapter, the term preparedness is described and a quantitative measure for calculating the preparedness for the fire and rescue service is developed. The development and validation process of the preparedness measure is described. To measure the preparedness, an integer programming (IP) problem has to be solved in order to determine the resources that are most suited to contribute to the preparedness. It has been shown that the IP-problem can be solved 
efficiently by a simple greedy based heuristic, which found optimal solutions in $95 \%$ of the cases that were tested. The used heuristic has thus been deemed good enough for measuring the preparedness in various situations.

The developed preparedness measure has been validated and calibrated using preparedness evaluations made by professional staff from the fire and rescue service in Sweden. In the validation and calibration process, the measured preparedness has been compared with the evaluations i.e. how the preparedness is perceived by professional staff from the fire and rescue service.

The evaluated results show that there is a lot of variation in how the fire and rescue service personnel perceive the preparedness. However, threshold values to specify if the calculated preparedness is good or inadequate were found in the calibration process. The validation results indicate that it is possible to find threshold values so that the measured preparedness correspond to the fire and rescue service experts' perceived preparedness in approximately $60 \%$ of the cases that were examined. The results also show that the experts do not agree with each other all the time. From the evaluations, it is clear that the fire and rescue service personnel perceive the preparedness differently and sometimes as a quite fuzzy concept. Opinions about what constitute good or inadequate preparedness also vary even between close colleagues. A preparedness measure like the one developed in this chapter might help to reduce the fuzziness. If the measure for example should be implemented in a geographical information system, it should become clear at least how the measure calculates the preparedness, and in time, the users might adopt this view. Thus, the measure could facilitate a common ground for situational awareness between individual decision makers.

The developed preparedness measure can be used as a basis in various decision support tools in order to e.g. facilitate resource planning and decision making. Such support tools are presented in Chapter 7. It should also be possible to directly use the developed measure as a base for planning and decision making. For example, a good view of an area can be obtained just by observing where the preparedness is good or inadequate. The dispatcher can then refrain from moving resources away from areas with inadequate preparedness. Resources can instead be dispatched from areas with good preparedness, or relocated from areas with good preparedness to areas with inadequate preparedness if that should be necessary. 


\section{Relocation of Fire and Rescue Resources}

The planning process of fire and rescue resources sometimes includes relocations of resources. It can be necessary if for example many resources in an area are busy and the area is lacking available resources for new incoming alarms. Resources may then be moved to, or closer to, these areas. Since the need for assistance also varies with time, it may sometimes be essential to relocate resources to other fire stations or potential assembly points for being standby where the need for assistance has increased. In some situations, it may also be necessary to call in part-time firefighters to their home fire station. In such cases, firefighters are called in to the station (or any other location) for being in a standby position for new incidents.

When relocating resources, important issues are to determine which resources to relocate and decide where to relocate them. It is not always obvious if and how resources should be relocated or called in to a station or another position for being standby. The purpose of relocating resources is mainly to maintain a good level of preparedness, as discussed in Chapter 5. According to the Swedish fire and rescue services many parameters are considered if and when resources should be relocated. For example it depend on the ongoing accidents, the expected risk situation, which resources that currently are available and those that currently are busy, where the resources are located and the expected time until the busy resources again are available. Usually, relocations are made when resources are expected to be busy for quite some time. When the fire and rescue services in Sweden relocate resources, they usually move them to other fire stations or predefined break points, often strategic places that are easy to reach e.g. intersections. However, the predefined break points are not optimally chosen and sometimes there might be other places that are more suitable to use for relocations than the predefined, depending on the situation and the purpose of the relocation. Since it is a challenge to determine which resources to relocate and where to relocate them, it might facilitate if the fire and rescue service can get some kind of support in the decision making.

In this chapter, a model to relocate fire and rescue resources is presented. The model gives suggestion on which resources to relocate and where to relocate them, in a given area. The objective is to give fire and rescue services support in their resource planning process and to improve the preparedness, if that is possible, in the given area.

\subsection{A Resource Relocation Model}

The resource relocation model, presented in this section, gives suggestions of how vehicles and firefighters can be relocated in order to improve the preparedness for expected accidents in a given area. The preparedness level is measured according to the developed measure in Section 5.1.4. The model considers the preparedness for multiple types of accidents and the resources that are required to handle the accidents. Thus, the model also considers different types of vehicles and firefighters as well as if there are any conditions for the resources, e.g. that a certain type of vehicle only can be handled by a particular type of firefighters. 
To improve the preparedness, it can also be necessary to call-in part-time firefighters to stations or other positions, since they usually have a longer preparation time than full time firefighters. Therefore, both relocations and call-ins of firefighters are considered in the model.

If resources should be suggested to be relocated to new places or called in to specific positions, the relocations must be cost-effective. In order to avoid unnecessary relocations or call-ins of firefighters, three types of costs are included in the model. There is a fixed cost for relocating a vehicle, a variable cost for relocating freighters and a variable cost for calling in part-time firefighters. The fixed cost is paid for each relocated vehicle regardless the distance of the move. The cost for relocating a firefighter is paid per time-unit of the travel time from the firefighters' initial positions. The cost for calling in a part-time firefighter to be in a stand by position is paid per time-change in the preparation time. The costs are specified in preparedness units. A move is cost-effective if it improves the preparedness despite the costs.

The objective of the model is to suggest how resources can be relocated or called-in to specific places in order to improve the preparedness for all included types of accidents. However, when improving the preparedness in some zones, it may get worse in others. To avoid improving the preparedness in zones at the same time as zones with an inadequate preparedness should get an even worse preparedness; the objective function of the model is to minimize the sum of the preparedness over the current $N$ zones with the worst preparedness. $N$ is set to an appropriate number of zones regarding the total number of zones included in the problem. Furthermore, the costs must also be included in the objective function since relocations only are profitable if they improve the preparedness while the costs are taken into account.

Let the given area be divided into a number of zones. The model is then formulated as follows:

Sets:

$I=$ the set of zones

$J=$ the set of zones

$A=$ the set of accident types

$U=$ the set of vehicles

$P=$ the set of persons

$K=$ the set of vehicle types

$R=$ the set of personnel competencies

\section{Variables:}

$x_{\text {praj }}=\left\{\begin{array}{l}1, \text { if person } p \text { contributes with competency } r \\ \text { to the preparedness for accident } a \text { in zone } j \\ 0, \text { otherwise }\end{array}\right.$

$y_{u a j}=\left\{\begin{array}{l}1, \text { if vehicle } u \text { contributes to the preparedness for accident } a \text { in zone } j \\ 0, \text { otherwise }\end{array}\right.$ 
$z_{\text {puaj }}=\left\{\begin{array}{l}1, \text { if person } p \text { is assigned to vehicle } u \text { to accident } a \text { in zone } j \\ 0, \text { otherwise }\end{array}\right.$

$e_{p u i}=\left\{\begin{array}{l}1, \text { if person } p \text { and vehicle } u \text { is located in zone } i \\ 0, \text { otherwise }\end{array}\right.$

$f_{\text {puaj }}=\left\{\begin{array}{l}1, \text { if person } p \text { drives vehicle } u \text { to accident } a \text { in zone } j \\ 0, \text { otherwise }\end{array}\right.$

$g_{p i j}=\left\{\begin{array}{l}1, \text { if person } p \text { relocates from zone } i \text { to zone } j \\ 0, \text { otherwise }\end{array}\right.$

$h_{u i j}=\left\{\begin{array}{l}1, \text { if vehicle } u \text { relocates from zone } i \text { to zone } j \\ 0, \text { otherwise }\end{array}\right.$

$n_{p i}=\left\{\begin{array}{l}1, \text { if person } p \text { is located in zone } i \\ 0, \text { otherwise }\end{array}\right.$

$m_{u i}=\left\{\begin{array}{l}1, \text { if vehicle } u \text { is located in zone } i \\ 0, \text { otherwise }\end{array}\right.$

$w_{j}=\left\{\begin{array}{l}1, \text { if } j \text { is one of the } N \text { worst zones } \\ 0, \text { otherwise }\end{array}\right.$

$\tilde{A}_{p}=$ New preparation time for person $p$

$P_{a j}=$ the preparedness values for accident $a$ in zone $j$

$\rho_{j}=$ the sum of preparedness values over all the accident types in zone $j$, if $j$ is one of the $N$ worst zones

$V_{p a j}=$ the response time for person $p$ to accident $a$ in zone $j$ if person $p$ is requested

$T_{u a j}=$ the response time for vehicle $u$ to accident $a$ in zone $j$ if vehicle $u$ is requested

\section{Parameters:}

$F_{p i}=\left\{\begin{array}{l}1, \text { if zone } i \text { is person } p \text { 's initial position } \\ 0, \text { otherwise }\end{array}\right.$

$L_{u i}=\left\{\begin{array}{l}1, \text { if zone } i \text { is vehicle } u \text { 's initial position } \\ 0, \text { otherwise }\end{array}\right.$

$G_{p u}=\left\{\begin{array}{l}1, \text { if person } p \text { can drive vehicle } u \\ 0, \text { otherwise }\end{array}\right.$

$Q_{p r}=\left\{\begin{array}{l}1, \text { if person } p \text { posesses competency } r \\ 0, \text { otherwise }\end{array}\right.$

$S_{u k}=\left\{\begin{array}{l}1, \text { if vehicle } u \text { is of type } k \\ 0, \text { otherwise }\end{array}\right.$

$W_{u}=$ the number of seats available in vehicle $u$

$t_{i j}=$ travle time from zone $i$ to zone $j$

$a_{p}=$ preparation time for person $p$

$B_{r a}=$ required number of persons with competency $r$ to accident $a$

$D_{k a}=$ required number of vehicles of type $k$ to accident $a$ 
$d_{a j}=$ expected number of accidents $a$ in zone $j$

$C=$ cost for moving a vehicle

$K=$ cost for moving a person per unit of travel time from the persons initial position

$c=$ call in cost for a part time person per change in preparation time

$E=$ the standard prepartaion time for a full time firefighter

$N=$ a selected number of zones with the worst preparedness

$M=$ a very large number

$\gamma=$ parameter related to the expected demand

Minimize $\sum_{j \in J} \rho_{j}+\sum_{i \in I} \sum_{j \in J} \sum_{u \in U} C h_{u i j}+\sum_{i \in I} \sum_{j \in J} \sum_{p \in P} K g_{p i j} t_{i j}+\sum_{p \in P} c\left(a_{p}-\tilde{A}_{p}\right)$

Subject to

$\sum_{i \in I} n_{p i}=1$

$\forall p \in P$

$\sum_{i \in I} m_{u i}=1$

$\forall u \in U$

$\sum_{p \in P} x_{p r a j} \geq B_{r a}$

$\forall r \in R, a \in A, j \in J$

$\sum_{u \in U} S_{u k} y_{u a j} \geq D_{k a}$

$\forall k \in K, a \in A, j \in J$

$\sum_{p \in P} z_{\text {puaj }} \leq W_{u} \sum_{p \in P} f_{\text {puaj }}$

$\forall u \in U, a \in A, j \in J$

$\sum_{p \in P} z_{\text {puaj }} \geq y_{u a j}$

$\forall u \in U, a \in A, j \in J$

$\sum_{u \in U} z_{p u a j} \geq \sum_{r \in R} x_{p r a j}$

$\forall p \in P, a \in A, j \in J$

$\sum_{u \in U} z_{p u a j} \leq 1$

$\forall p \in P, a \in A, j \in J$

$\sum_{r \in R} x_{p r a j} \leq 1$

$\forall p \in P, a \in A, j \in J$

$x_{\text {praj }} \leq Q_{p r}$

$\forall p \in P, r \in R, a \in A, j \in J$

$\sum_{p \in P} f_{\text {puaj }} \leq 1$

$\forall u \in U, a \in A, j \in J$

$\sum_{u \in U} f_{\text {puaj }} \leq 1$

$\forall p \in P, a \in A, j \in J$ 


$$
\begin{aligned}
& f_{\text {puaj }} \leq z_{\text {puaj }} \\
& \forall p \in P, u \in U, a \in A, j \in J \\
& 2 * e_{p u i} \leq n_{p i}+m_{u i} \\
& \forall p \in P, u \in U, i \in I \\
& f_{\text {puaj }} \leq G_{p u} \\
& \forall p \in P, u \in U, a \in A, j \in J \\
& z_{\text {puaj }} \leq \sum_{i \in I} e_{\text {pui }} \\
& \forall p \in P, u \in U, a \in A, j \in J \\
& g_{p i j} \leq \sum_{u \in U} h_{u i j} \\
& h_{u i j} \leq \sum_{p \in P} g_{p i j} \\
& \sum_{p \in P} g_{p i j} \leq \sum_{u \in U} h_{u i j} W_{u} \quad \forall i \in I, j \in J \\
& \sum_{j \in J} g_{p i j} \leq F_{p i} \\
& \forall p \in P, i \in I \\
& \sum_{i \in I} g_{p i j} \leq n_{p j} \\
& \forall p \in P, j \in J \\
& \sum_{j \in J} h_{u i j} \leq L_{u i} \\
& \sum_{i \in I} h_{u i j} \leq m_{u j} \\
& \tilde{A}_{p} \geq E \\
& \tilde{A}_{p} \leq a_{p} \\
& \tilde{A}_{p} \leq a_{p}-\left(a_{p}-E\right)\left(F_{p i}-n_{p i}\right) \\
& \sum_{j \in J} w_{j}=N \\
& P_{a j}=d_{a j} \gamma\left(\frac{\sum_{p \epsilon P} V_{p a j}+\sum_{u \in U} T_{u a j}}{\sum_{r \in R} B_{r a}+\sum_{k \in K} D_{k a}}\right) \\
& \forall p \in P, u \in U, a \in A, j \in J \\
& \rho_{j}+M\left(1-w_{j}\right) \geq \sum_{a \in A} P_{a i}-M w_{i} \\
& \forall i \in I, j \in J \\
& \rho_{j}+M\left(1-w_{j}\right) \geq \sum_{a \in A} P_{a j} \\
& \forall j \in J \\
& T_{u a j} \geq\left(t_{i j}+\tilde{A}_{p}\right)+M\left(m_{u i}+z_{p u a j}+y_{u a j}-3\right) \quad \forall u \in U, p \in P, a \in A, i \in I, j \in J
\end{aligned}
$$




$$
\begin{array}{ll}
V_{\text {paj }} \geq t_{i j}\left(n_{p i}+\sum_{r \in R} x_{\text {praj }}-1\right) & \\
\quad+\tilde{A}_{q}+M\left(z_{\text {puaj }}+\sum_{r \in R} x_{\text {praj }}+z_{\text {quaj }}-3\right) & \\
\tilde{A}_{p}, T_{u a j}, V_{\text {paj }}, P_{a j}, \rho_{j} \geq 0 & \\
x_{\text {praj }}, y_{u a j}, z_{\text {puaj }}, e_{\text {pui }}, f_{\text {puaj }}, g_{p i j}, h_{u i j,}, \quad \forall p \in P, u \in U, a \in A, j \in J \\
n_{p i}, m_{u i}, w_{j} \in\{0,1\} & \forall p \in P, r \in R, u \in U, a \in A, \\
& k \in K, i \in I, j \in J
\end{array}
$$

The objective function (1) is the sum of the total preparedness, i.e. for all types of accidents included in the problem, for the $N$ zones with the worst preparedness, plus the costs for the relocations of resources (vehicles and firefighters). The types of accidents $a$ can be ignored in the model if only one type of accident should be considered.

Constraint (2) and Constraint (3) in the model ensures that all available persons and vehicles included in the problem are located to exactly one zone. Constraint (4) in the model ensures the minimum manning level of personnel competencies that are required for a certain type of accident, and Constraint (5) ensures the need of a certain type of vehicles requested to the accident type. A vehicle only has a specified number of seats available, therefore only a maximum number of persons can be assigned to each specific vehicle. Constraint (6) ensures that the vehicle seating capacity is not exceeded, and it also ensures that each responding vehicle has a driver that can drive the vehicle to the accident site. Constraint (7) ensures that there always must be at least one person assigned to a vehicle if it should be possible to use the vehicle. Then, for a person to be able to contribute to the preparedness for an accident there must be a vehicle available to transport the person to the accident site. That condition is ensured by Constraint (8).

Furthermore, a person can be assigned to only one vehicle, only one of a person's competencies can be utilized at a time, and a person can only contribute with a certain competency if the person possesses the competency. These conditions and restrictions are ensured by Constraints (9), (10) and (11). A vehicle can only be driven by one person at a time, and a person can only drive one vehicle at a time. This is ensured by Constraints (12) and (13) respectively. The person who drives a vehicle must also be assigned to that vehicle. That condition is ensured by Constraint (14). Constraint (15) is included in the model to determine if a person and a vehicle is located in the same zone. Since persons sometimes must have specific qualities or skills to be able to drive certain vehicles, Constraint (16) is included in the model. This condition ensures that a person can drive a certain vehicle only if the person is permitted and allowed to drive the vehicle. Constraint (17) ensures that a person only can be transported in a vehicle if both the person and the vehicle are located in the same zone. 
If a person should be relocated from one zone to another, at least one vehicle must also be relocated in order to transport the person from its current location to the new location. If instead a vehicle should be relocated, at least one person, able to drive the vehicle, must also be relocated. These two conditions are ensured by Constraints (18) and (19). For all persons that should be relocated, there must be enough vehicles to transport the persons. That condition is ensured by Constrains (20). If a resource (person or vehicle) should be relocated, the resource's initial position is equal to the position the resource is relocated from, and the position the resource is relocated to is the resource's current position. These conditions are represented by Constraints (21) and (22) for the personnel resources and by Constraints (23) and (24) for the vehicles.

If a person should be relocated, the person's preparation time may sometimes be changed. The preparation time is never less than a standard preparation time of $E$ seconds, and that is represented by Constraint (25). If a person relocates, the preparation time will always change to $E$ seconds because the person is then in a stand by position able to respond to accidents just like a full time firefighter. The change in preparation time when a person is relocated is ensured by Constraint (27). Constraint (26) ensures that a person's preparation time never will be greater than the person's initial preparation time which is never less that $E$ seconds.

Constraint (28) ensures that the sum of the number of zones with the worst preparedness is equal to $N$ zones. $N$ is declared by the user. Constraint (29) represents the preparedness, as defined in Chapter 5, for an accident in each zone and Constraints (30) and (31) determines the $N$ zones with the worst preparedness. Constraints (32) and (33) represent the response time for vehicles and persons that contribute to the preparedness. The response time for a vehicle is the vehicle's travel time plus the preparation time for the person assigned to the vehicle with the longest preparation time. The response time for a person is the person's travel time plus the preparation time for the person assigned to the same vehicle who has the longest preparation time.

\subsection{Solution Method for the Relocation Model}

The developed model has been tried to solve by Gurobi Optimizer 5.6.0 by using a PC laptop, with an Intel Core i5-2520M, $2.50 \mathrm{GHz}$ processor, and $8 \mathrm{~Gb}$ RAM. The model has only been tried to solve for a small problem containing 30 demand zones, 17 different firefighters and 4 vehicles. No optimal solution has been found within 5 hours. A feasible solution has been found within 2 hours, however, with a gap of $100 \%$. It is thus considered complicated to exactly solve the model. If a solution should be found in more than 5 hours, the solution time would nonetheless be unacceptably long. Since a quick solution time is desired for the resource relocation problem, a heuristic is developed for solving the model within a reasonable time.

\subsubsection{The Heuristic Developed for Solving the Relocation Model}

The heuristic used for solving the relocation model, i.e. to determine how resources can be relocated to improve the preparedness, is based on a greedy randomized search procedure. The search procedure starts from an initial state (a feasible solution) and is then searching for a new candidate solution by relocating resources in order to improve the initial solution. 
During the search phase a candidate solution is randomly generated with respect to a number of zones $n$ with the worst measured preparedness. The number of zones $n$ that are considered is defined by the user and should be selected such that $n<N$. If a new candidate solution $x$ ' is better than the current best found solution $x$, the search moves to the new solution $x$, and then, the new solution $x^{\prime}$ will be the current best solution $x$. If $x^{\prime}$ is not a better solution, $x$ is kept as the best solution and a new candidate solution will randomly be generated. The search procedure continues for a predefined number of iterations.

The preparedness must frequently be calculated for the current state to determine if a better solution is found or not. In order to calculate the preparedness, the measure defined in Section 5.1.4 is used and the solution process described in Section 5.3.1 is used for solving the resource selection problem.

Initially in the developed heuristic, a checkup zone is randomly selected in each iteration in the search phase. The checkup zone is one of the $n$ zones with the worst preparedness. The checkup zone is selected to determine which resources should be tested for relocations and also to determine which position (geographically) to relocate them to.

Generating a new random solution in the search process consists of selecting a number of resources, i.e. vehicles and persons that should be tested for relocations. The resources can either be relocated to another location or, if it is a personnel resource, it can also be called in for being standby at its designated home fire station or any other suitable place within the area. However, it is only personnel resources with a preparation time greater than $E$ seconds that can be called in to a fire station, i.e. part-time firefighters.

The resources that are tested for relocation are randomly selected of the resources that are contributing (selected by the resource selection model described in Chapter 5) to the preparedness for the accidents in the checkup zone, i.e. a random number of the resources are selected. The resources to be tested are stored in a list, $L_{\text {Resources. }}$ Of all the selected resources in the list $L_{\text {Resources }}$, either all or none will be relocated. Notice that a personnel resource cannot be relocated without a vehicle that can transport the person, and similarly, a vehicle cannot be relocated without a person who can drive the vehicle. The selected resources in the list $L_{\text {Resources }}$ are tested to be relocated to a new position in order to improve the preparedness in the $N$ zones with the worst measured preparedness. 


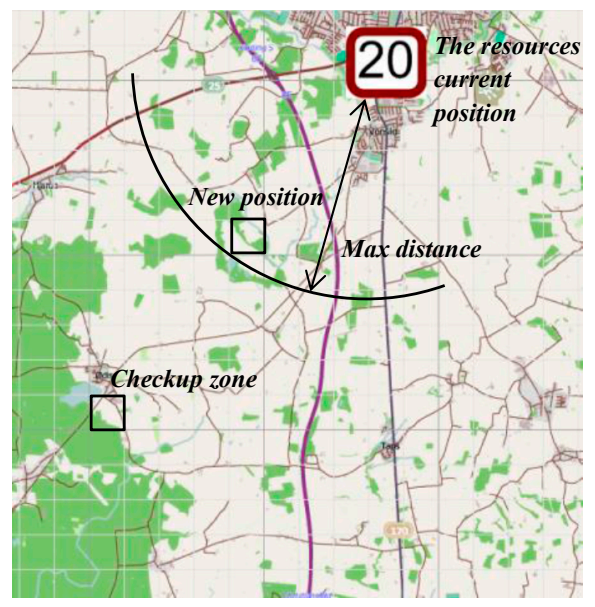

Figure 14. Illustration of the checkup zone and the new position.

When resources are tested for relocation, the new position will be found within an area around the resources' current position, i.e. within a certain distance or travel time from the resources' current position. The area includes all the places to which the resources can be relocated. In the search procedure, the new position is the position in the predefined area that is closest to the current checkup zone; see an illustration in Figure 14, the selected resources that contribute to the preparedness in the checkup zone are currently located at Station 20 in the figure. The preparedness will then likely be improved if resources would be located closer to the checkup zone.

Relocating resources also include some costs. Relocating a vehicle entails a fixed cost $C$, while there is a variable cost for relocating a personnel resource. The cost $K$ will be paid per unit of the travel time $t_{i j}$ for moving a person from zone $i$ to zone $j$, i.e. the relocation cost for a person $=K * t_{i j}$. There is also a cost $c$ per changed time unit in the preparation time $\left(a_{p}-\widetilde{A}_{p}\right)$ for the firefighters who should be called in for standby. When a firefighter gets called in, the preparation time is always changed to the standard preparation time of $E$ seconds, i.e. $\tilde{A}_{p}=E$.

The reason that resources relocates within an area around the resources current position is to avoid too costly relocations in just one step. Resources can, however, be relocated stepwise to positions further away. During the iterations, it is possible for resources to be relocated several times. In each iteration in the search process (when resources are tested for relocation) the area the resources can be relocated within changes depending on the resource's position. In the end, it is only the move and the cost for relocating the resources from their initial position to the end position that are considered and paid for. If the heuristic should suggest relocations of resources, i.e. if a better solution is found (if $x$ ' is better than $x$ ), the relocations of the resources and the cost for relocating them must result in an improvement in the preparedness for the current $N$ zones with the worst preparedness. The pseudo code for the developed heuristic is presented in Table 20. 
Table 20. Pseudo Code for the heuristic developed for solving the relocation model.

The pseudo code for the heuristic for solving the relocation model

Let $x=$ the current best found solution

Let $x^{\prime}=$ a new candidate solution
Let $z=$ the best known overall solution

Let $\mathrm{y}=$ the solution to the initial state

Let $n$ be a selected number of zones with the worst preparedness

Let TotalNumberOfIterations $=0$

Let $z=y$

While (TotalNumberOfIterations < MaxTotalNumberOfIterations)

ImprovmentIterations $=0$

Iterations $=0$

$x=y$

While (ImprovmentIterations < MaxImprovementIterations \&\& Iterations < MaxNumberOfIterations)

NewSolution $=$ false

Randomly select a Checkup Zone from $n$.

Randomly select a number of firefighters and vehicles that contribute to the preparedness in the checkup zone and store them in $L_{\text {Resources }}$

Foreach (firefighter $f$ in $L_{\text {Resources }}$ )

If (firefighter $f$ has a preparation time $>E$ )

Let the firefighter be added to a list $L_{\text {Firefighters }}$

Let the preparation time for the firefighters in the list $L_{\text {Fire fighters }}=E \rightarrow x^{\prime}$

Evaluate $x^{\prime}-$ Entails calculating the preparedness

If $(x$ ' is better than $x)$

$$
\begin{aligned}
& x=x \\
& \text { NewSolution = true }
\end{aligned}
$$

Relocate the firefighters and the vehicles in the list $L_{\text {Resources }}$ to the new position $\rightarrow x$, Evaluate $x$ ' - Entails calculating the preparedness

If $(x$ ' is better than $x)$

$$
\begin{aligned}
& x=x \\
& \text { NewSolution = true }
\end{aligned}
$$

If $($ NewSolution $=$ true $)$

$$
\text { ImprovmentIterations }=0
$$

Else

$$
\text { ImprovmentIterations = ImprovmentIterations }+1
$$

Iterations $=$ Iterations +1

Let $z^{\prime}=$ the best found solution $x$

If $(z$ ' is better than $z)$

$$
z=z^{\prime}
$$

TotalNumberOfIterations $=$ TotalNumberOfIterations +1

$z=$ the best found solution 


\subsubsection{Test Scenarios}

To verify the relocations suggested by the developed heuristic, the relocation model has heuristically been solved for a number of scenarios of the same test setting as explained in Chapter 5. When solving the relocation problem, the three types of expected accidents, which include traffic accidents, building fires and fires in high-rise buildings, were considered. The accident types are further explained in Chapter 5. The objective was, thus, to improve the preparedness for these three types of accidents.

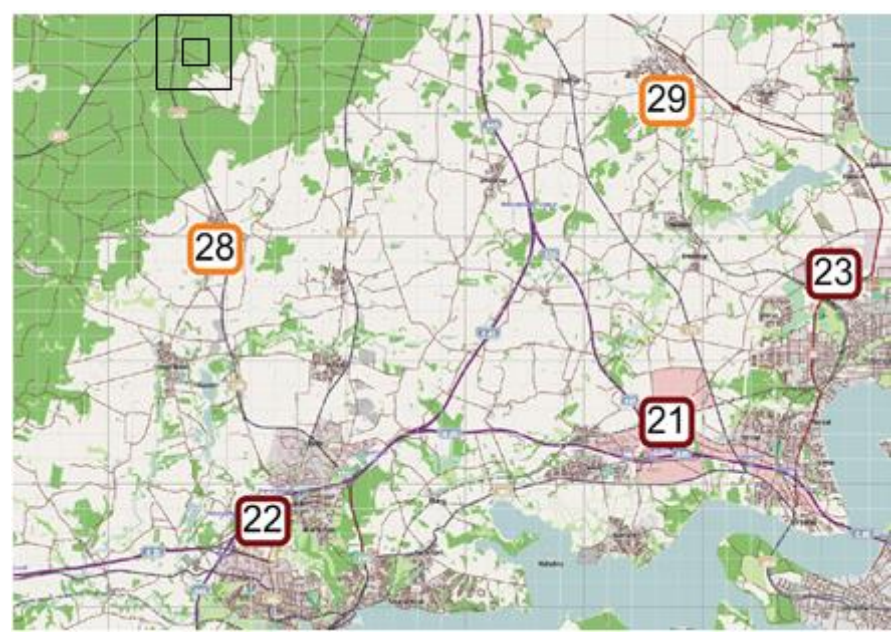

Figure 15. An illustration of how nine normal demand zones are aggregated to a large demand zone.

The number of demand zones for the test setting is 2584 . When solving the relocation problem, these demand zones were aggregated to 305 demand zones in order to reduce the solution time, which had proven to be unacceptably long since it takes around 7-10 seconds to measure just the preparedness for 2584 demand zones; see Section 5.4. The preparedness must be measured approximately three times per iteration when heuristically solving the relocation problem. For 300 iterations in the solution process, it means it will take around 1.75 to 2.5 hours. Therefore, the demand zones were aggregated to 305 . In the aggregation process, nine "normal" demand zones form a larger demand zone. An illustration is shown in the upper left corner on the map in Figure 15. The little zone in the middle represents a "normal" demand zone, and the larger square represents a large demand zone formed by nine normal zones.

In the solution process, the travel time in which resources can be relocated within in each iteration is set to 20 minutes. This means, during the process, resources can be relocated in steps of a maximum 20 minutes of travel time. A total of 20 minutes was considered as a suitable travel time in order to preclude significantly high travel costs. Further, $N=30$ and $n=$ 10. This means the checkup zone is randomly selected from the current 10 zones with worst preparedness and a new solution $x^{\prime}$ is better than the current best found solution $x$ if the relocations and the costs for the relocations result in an improvement in the preparedness for the 30 zones with the worst preparedness. 
The various costs for relocating resources have been settled by analyses performed with the input of the fire and rescue service in Sweden. The cost $C$ used for relocating a vehicle is set to 25 units of preparedness, which reflects the costs associated with moving a vehicle from its initial position to its final position. For relocating a person, the cost $K$ is set to 0.48 units of preparedness per minute (travel time) the person is moved from its initial position. The call-in cost $c$ for a part-time person (for being in a normal standby position) is 1.8 units of preparedness per change in minutes of the person's preparation time.

The solution time for solving the relocation problem with the developed heuristic depends on both the number of iterations in the search procedure and on the number of demand zones included in the problem. For the test scenarios, the maximum number of iterations was 300 . If no better solution is found within 30 iterations, a round is completed and the best found solution is stored as a new candidate solution $z$ ' to the problem. If $z$ ' is better than the best found solution $z, z$ ' will be the best found solution $z$. A new round will thereafter start from the initial state. The process continues until at least 300 iterations have been performed. The best found solution $z$ from all the rounds is the final result from the solution process. A test scenario with 305 demand zones takes around 1 minute to solve when using a PC laptop, with an Intel Core i5-2520M, 2.50 GHz processor and $8 \mathrm{~Gb}$ RAM.

To analyze suggestions of resource relocations produced by the heuristic, the relocation problem is solved 10 times for 18 scenarios (the same scenarios that were analyzed in chapter 5) of the test setting. A total of 10 suggestions of resource relocations have, thus, been produced for each one of the scenarios. Improved solutions are found in all 18 scenarios.

\subsubsection{Computational Results}

To investigate the improvements of the produced solutions, they have been compared to the corresponding initial state for each one of the scenarios. For each scenario, Table 21 presents the difference in percent between the average values from the 10 produced solutions (when the suggested relocations are implemented) and the corresponding value for the initial state (when resources not are relocated) for the specific scenario.

The results in the table consider all 305 demand zones in the test area except the results in the third column, which only consider the 30 zones with the worst preparedness. At the bottom of the table, the total average values for all scenarios are presented. A negative value in the table indicates declining preparedness if the suggested relocations are implemented compared to if they are not implemented.

The second column presents the improvement of the preparedness for all considered accident types (i.e. the sum of the preparedness for expected building fires (BF), traffic accidents (TA) and fires in high-rise buildings (HBF) in all demand zones). Correspondingly, the next column presents the improvement of the preparedness for the considered accident types for only the 30 zones with the worst preparedness. The next three columns present the improvement of the average response time to expected accidents of each type. The response time means the time until all the requested resources (for the specific accident type) have reached the accident site. The last three columns present the improvements of the maximum 
preparedness value for each one of the accident types. In all scenarios, the preparedness is measured for all included zones. The maximum value is then found in the zone that has the greatest measured preparedness value. Since a low preparedness value is considered as good; see Section 5.1.4. Reduction of the maximum preparedness is desirable, which is indicated by a positive value in the table.

Table 21. Average improvements (in \%) when solving the resource relocation problem with the heuristic.

\begin{tabular}{|c|c|c|c|c|c|c|c|c|}
\hline Scenario & $\begin{array}{l}\text { Total } \\
\text { impr. }\end{array}$ & $\begin{array}{l}\text { Impr. } \\
\text { for the } \\
\text { 30 } \\
\text { worst } \\
\text { zones }\end{array}$ & $\begin{array}{l}\text { Impr. of } \\
\text { the average } \\
\text { response } \\
\text { time to } \\
\text { building } \\
\text { fires }\end{array}$ & $\begin{array}{l}\text { Impr. of } \\
\text { the average } \\
\text { response } \\
\text { time to } \\
\text { traffic } \\
\text { accidents }\end{array}$ & $\begin{array}{l}\text { Impr. of the } \\
\text { average } \\
\text { response } \\
\text { time to high- } \\
\text { rise building } \\
\text { fires }\end{array}$ & $\begin{array}{l}\text { Impr. of the } \\
\text { maximum } \\
\text { preparedness } \\
\text { value for } \\
\text { building fires }\end{array}$ & $\begin{array}{l}\text { Impr. of the } \\
\text { maximum } \\
\text { preparedness } \\
\text { value for } \\
\text { traffic } \\
\text { accidents }\end{array}$ & $\begin{array}{l}\text { Impr. of the } \\
\text { maximum } \\
\text { preparedness } \\
\text { value for fires } \\
\text { in high-rise } \\
\text { buildings }\end{array}$ \\
\hline 1 & 9 & 16 & 9 & 9 & -1 & 33 & 9 & 0 \\
\hline 2 & 23 & 26 & 22 & 22 & 18 & 24 & 14 & 14 \\
\hline 3 & 9 & 14 & 10 & 10 & 1 & 15 & 7 & 1 \\
\hline 4 & 3 & 8 & 3 & 3 & 3 & 16 & 13 & 9 \\
\hline 5 & 3 & 4 & 2 & 2 & 5 & 0 & 4 & 0 \\
\hline 6 & 6 & 10 & 6 & 6 & 4 & 24 & 9 & 0 \\
\hline 7 & 10 & 16 & 11 & 11 & -9 & 22 & 10 & 0 \\
\hline 8 & 10 & 14 & 10 & 10 & 13 & 8 & -1 & 3 \\
\hline 9 & 16 & 22 & 16 & 16 & 7 & 22 & 20 & 6 \\
\hline 10 & 11 & 17 & 12 & 12 & -2 & 16 & 13 & 14 \\
\hline 11 & 7 & 16 & 8 & 8 & 7 & 21 & 12 & 20 \\
\hline 12 & 13 & 20 & 13 & 13 & -4 & 21 & 15 & 0 \\
\hline 13 & 8 & 15 & 7 & 7 & 11 & 28 & 19 & 7 \\
\hline 14 & 4 & 10 & 5 & 5 & -7 & 18 & 0 & 0 \\
\hline 15 & 13 & 17 & 14 & 14 & 6 & 22 & 7 & 11 \\
\hline 16 & 9 & 13 & 9 & 10 & -1 & 13 & 5 & 0 \\
\hline 17 & 11 & 17 & 12 & 12 & -12 & 16 & 6 & -19 \\
\hline 18 & 15 & 21 & 15 & 15 & 1 & 20 & 11 & -4 \\
\hline Total & 10 & 15 & 10 & 10 & 2 & 19 & 9 & 3 \\
\hline
\end{tabular}

The results show for all scenarios an improvement in both the preparedness across all demand zones and the preparedness for the 30 zones with the worst preparedness. The average improvement of the preparedness for all scenarios is $10 \%$ for the total preparedness and $15 \%$ for the preparedness of the 30 zones with the worst preparedness.

If comparing the results for the different types of accidents, it must also be noted that all the demand zones do not contain a forecast of expected accidents for all accident types. A total of 290 demand zones contain forecasts for TA, 284 contain forecasts for BF and 18 contain 
forecasts for HBF. Also, a demand zone never contains forecasts for both BF and HBF. The results show that the average response time to $\mathrm{HBF}$ deteriorates in seven scenarios while improving the average response time for $\mathrm{BF}$ and $\mathrm{TA}$ in the same scenarios. Also, the maximum preparedness values deteriorate in two scenarios for HBF, while the values mostly improve for $\mathrm{BF}$ and $\mathrm{TA}$. Improving the preparedness for $\mathrm{BF}$ and $\mathrm{TA}$ seems to be prioritized compared to improving the preparedness for $\mathrm{HBF}$. The preparedness for $\mathrm{HBF}$ may also already be good enough from the beginning and it may therefore be difficult to improve it. $\mathrm{HBF}$ are also only expected in urban areas, where the majority of available resources are usually located. Another factor that can affect the results could be the additional demand for resources requested for HBF; see Section 5.4.1. Also, that there are fewer HBF-62 expected $\mathrm{HBF}$ compared to 312 expected TA and 154 expected $\mathrm{BF}$ - might affect the results. This might focus the model more on improving the preparedness for TA and BF. Moreover, the model will improve the preparedness for all considered accident types, and collectively, the improvement of the preparedness for all accident types is greater than the deteriorations - for all scenarios.

One of the main factors that affect the preparedness is the availability of resources. With only a few available resources, it is probably more important that they are well-positioned, than if a large number of resources are available. For example, if many resources are available, the probability that the resources already provide quite good coverage might be higher than if there only are a few resources available. Consequently, relocation of resources when many resources are available might not have as much of an impact as relocation of resources may have when only a few resources are available.

The scenarios differ in number of resources, competencies of the resources and how the resources are located; more explanation is provided in Section5.4. Scenarios containing few available resources, i.e. Scenarios 2, 8 and 9 (see the number of resources for each scenario in Table 17 in Section 5.4.3) show some of the greatest improvements of the preparedness values (Scenarios 2 and 9); see Table 21. Scenario 8 contains few resources, but shows less improvement than Scenarios 2 and 9. However, there are other scenarios that include more resources where the results also show great improvements, as in Scenarios 7, 10, 12, 15, 17 and 18. The number of available resources and also the type of available resources might somehow affect the results, but not as much as expected.

The main factor that influences the results, however, seems to be the resources' initial locations. If there are several zones with poor preparedness and if the resources are poorly located, there is greater potential for improvements than if the resources are well located and the preparedness is adequate in many zones from the beginning. Why the improvement of the preparedness is significantly higher in Scenario 2 compared to the improvements in the other scenarios is perhaps due to the fact that the preparedness is relatively poor in the initial state of Scenario 2. Therefore, the preparedness can be further improved in that scenario. For example, Scenario 2 has one of the worst (the highest) mean preparedness values in the initial state; see Table 17 in Section 5.4.3. 
For this study, it is, however, mostly the overall result that is interesting, i.e. that it is possible to relocate resources in order to improve the preparedness in an area. Furthermore, the developed resource relocation model can be solved using the developed heuristic and solutions (improvements of the preparedness) were found for all tested scenarios. But, as mentioned, the potential to improve the preparedness is contingent on each scenario, and the improvement seems to mostly depend on how the resources are initially located.

\subsubsection{Validation of the Developed Heuristic}

To validate the resource relocations suggested by the heuristic, a number of produced solutions have been evaluated by professional staff (experts) from different fire and rescue service in Sweden. The evaluation aims to determine whether the suggested resource relocations are realistic and reasonable for the fire and rescue service.

During the validation process, 16 experts from the fire and rescue service reviewed suggestions from a number of scenarios of the test setting (other scenarios than those presented in Table 21). The experts, in groups of two, evaluated the results from three or four different scenarios. In each scenario, the average preparedness (for all accident types) was improved both in total, i.e. for all demand zones as well as for the 30 worst zones, i.e. the zones with the worst preparedness.

The fire and rescue staff first inspected the initial state of each scenario, i.e. the state before suggestions were produced. Based on their own experience and thoughts, they evaluated the scenarios and how resources could be relocated to improve the preparedness in the corresponding area. Thus, they developed their own ideas that could be later compared to the produced suggestions. Thereafter, they were given access to the suggestions of relocations made by solving the relocation model for the specific scenario. From the results, they analyzed the relocations generated by the heuristic by considering how resources were relocated. They were told to observe the resources that were included in the relocations, the resources that were not included, the relocated resources' initial location and final destinations as well as how the relocations combined different resources (vehicles and firefighters). After observing the relocations, they were free to organize the resources as desired. They could, for example, follow the proposed suggestions, retain parts of the suggestions or organize the resources in a completely different way. 
Table 22. The validation results from the experts' evaluations of the resource relocation suggestions produced by the heuristic.

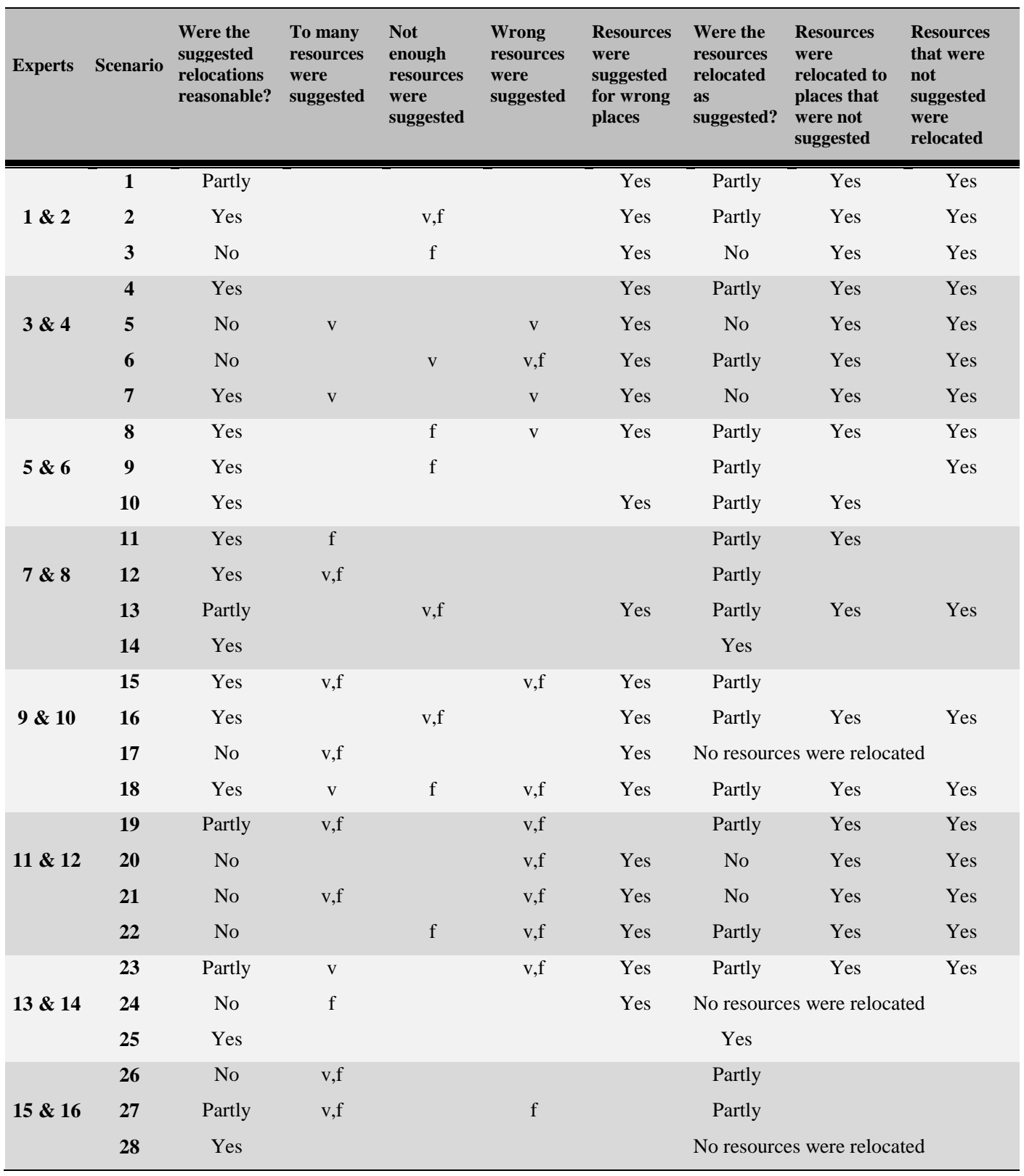

When the organization of resources was completed, the experts were given a questionnaire with questions about the suggested relocations. The purpose of the questions was to distill the experts' opinions and thoughts about the suggestions as well as how they were using them, i.e. if they followed the suggestions. The results from the questionnaire are summarized in 
Table 22. The first question was about whether the suggestions were reasonable according to certain situations in a specific scenario. They responded by answering yes, no or partly reasonable. They were also told to specify if they thought the suggestions included too many resources, too few resources or if incorrect/inappropriate resources were suggested for relocations. Depending on the answer, they were expected to specify the resource type, i.e. if it concerned vehicles (v) or firefighters (f) or both. Another question asked if they thought the resources were relocated to incorrect places, i.e. places the expert did not think were suitable to relocate resources to. The experts also answered if they used the suggestions or if they did not use them. They could also use parts of the suggestions. If they did not follow the suggestions or if they partly followed them, they were also told to specify if they relocated resources other than those suggested, if they relocated resources to places that were not suggested or if they did not relocate any resources at all. The experts also shared other comments and thoughts regarding the suggestions if they had any.

In Table 22, the results show that the experts, in 14 scenarios, i.e. $50 \%$ of the cases, thought that the produced relocation suggestions were reasonable, and in five scenarios, $18 \%$, they thought the suggestions were partly reasonable. This can be compared to the fact that the experts in around $70 \%$ of the cases used the suggestions, or parts of the suggestions. It was, however, only in two scenarios, Scenario 14 and Scenario 25, where the experts completely followed the suggestions. In one scenario, Scenario 7, the experts thought that the suggestions were reasonable, but they neither used the suggestion nor parts of it. The opposite occurred in three cases, in Scenarios 6, 22 and 26. In these scenarios, the experts did not think the suggestions were reasonable, but they still used parts of the suggestions. In eight scenarios, the experts did not use the suggestions or parts of them, and in three of these scenarios no resources were relocated at all.

In 19 of the scenarios, the experts thought that resources were suggested to be relocated to places that they considered incorrect. However, in almost $60 \%$ of these cases, the expert relocated the resources to a neighboring fire station instead of to the suggested positions. From the validation results, it becomes rather clear that it feels more natural and comfortable for the fire and rescue staffs to relocate resources to fire stations compared to other somewhat unknown positions. Even though the suggested resources were relocated to positions somewhat remote to a station, they often chose to relocate resources to the closest station. They also often mean that it is better for the firefighters to be relocated to stations, since they often have better working conditions at stations and there are also often additional resources available at stations, if necessary. Other reasons as to why the experts thought the resources were relocated to incorrect places, or why they relocated resources to places that were not suggested, were usually if resources were suggested to be relocated to areas in the countryside or if many single resources were suggested to be relocated to many different positions. In many cases, when the experts changed locations for the resources and relocated them to positions that were not suggested, they often relocated resources to strategic break points and positions close to a road, an intersection or between two fire stations. Also, the experts mostly preferred to relocate a group of at least two firefighters instead of relocating single firefighters. Therefore, sometimes the expert chose to relocate some resources in addition to 
the suggested. A reason why additional resources were sometimes relocated is that the expert did not think that the right resources were suggested from the beginning, or that too few resources were suggested. When the expert relocated additional firefighters, the motivation was normally not the firefighters' competencies, i.e. that a firefighter with a specific competency should be relocated. It was, rather, how the resources were geographically located and due to the ongoing accidents in the area. When the expert decided which vehicle to relocate, the vehicle type was always essential to the decision. If the expert thought too few resources were suggested for relocation, they usually thought that it was too few firefighters.

Another reason as to why the experts did not always follow the suggestions or only parts of them was that they thought that suggestions were unnecessary because they believed that some in-use resources would become available imminently. In such cases, they thought it was unnecessary to move resources closer to places with such resources. The suggested resources were then sometimes relocated to other positions or not relocated at all. Since the model does not consider this possibility, such as forecasting of ongoing accidents or whether resources will soon be available again, this is something the user must consider when planning and organizing the resources. This is also something that is inherently problematic to include in the resource relocation model since it is estimations made by the experts according to specific circumstances.

The validation results are based on persons' opinions and interpretations of different situations. Therefore, the results are influenced by the experts who evaluated the scenarios. In order to obtain as general an evaluation as possible, and also to avoid too much individual bias, the evaluations were performed by groups of two experts. The groups of experts also came from different fire and rescue services in Sweden.

\subsection{Conclusions}

In this chapter, it is shown that the developed resource relocation model can be solved using a greedy randomized search procedure. Obtained results indicate that the preparedness for traffic accidents, building fires and fires in high-rise buildings can be improved by relocating resources to new positions. The preparedness can be improved by up to $23 \%$ by relocating resources. On average in the test scenarios, the preparedness was improved by $10 \%$. If only $10 \%$ of the zones with the worst preparedness are considered, the preparedness can be improved by up to $26 \%$, and on average for the test scenarios the preparedness was improved by $15 \%$. The preparedness depends on both the demand for resources for the specific accident type as well as the forecast for the expected number of accidents for certain accident types. How much the preparedness can be improved depends on the availability of resources and the available resources' location. Therefore, the improvements in an area vary depending on the resources' origin.

The validation shows, in most cases, that the fire and rescue service staff thinks that the produced relocation suggestions are reasonable and usable. However, they did not completely follow the suggestions in more than two scenarios. In $64 \%$ of the cases, the fire and rescue service experts used parts of the suggestions. The main reason for changing the suggestions was that the experts would rather relocate resources to fire stations or other strategic places 
close to intersections or in between two fire stations instead of the suggested (sometimes rather unknown) locations in the area.

Another essential reason why the experts changed the produced suggestions was that these quite often consisted of relocations of only one firefighter and one vehicle, or that resources were suggested to be relocated to places quite far away in the countryside. Usually, if the experts chose to relocate resources, they wanted to relocate groups of at least two firefighters.

The purpose of the relocation model was to produce suggestions in order to support the staff, and offer recommendations and advice on how resources can be relocated to improve the preparedness for different types of accidents in a specific area; that is, the suggestions should support the resource-planning process. The staff should, thus, always make the final decisions about how resources should be allocated and whether they should be relocated in a specific situation. The evaluations also indicate that this is the way the produced suggestions are used.

In summary, improvements in preparedness have been observed in all tested scenarios. The produced suggestions for relocating resources are considered reasonable or partly reasonable in $68 \%$ of the cases and the suggestions are used or partly used in $71 \%$ of the cases. The suggestions produced by the developed relocation model are then considered useful and reasonable for the fire and rescue service staff in order to support the planning of resource coordinating. However, the suggestions could probably be improved if it, for example, would be possible to include estimations and forecasts about how resources will become available again soon. It could also be interesting to, for example, give the user the ability to influence which resources are available for relocations and how resources can be combined when relocating them. At present, these possibilities are not incorporated into the model. 


\section{Decision Support Tools for Fire and Rescue Services}

For fire and rescue services, in order to support efficient preparation for taking care of new incoming alarms and to apply dynamic planning; see Chapter 3, three decision support tools have been developed and are presented and described in this chapter. The first tool is a preparedness visualizer that can illustrates the preparedness for a given type of accident, the second gives suggestions on which resources that are most appropriate to dispatch to an accident in order to minimize the response time, and the third tool gives suggestions on how resources can be relocated in order to improve the preparedness, i.e. in order to best be available if an accident occur. The support tools are based on developed optimization models presented in Chapters 5 and 6.

The tools have been validated via experiments with a number of fire and rescue services in Sweden. In the experiments, fire and rescue service staff planned and managed resources dynamically with respect to various situations and demand of service. Via the experiments, the practical use of the support tools is also reviewed. The usefulness has been evaluated through questionnaires and comments from the participants in the experiments. This is discussed at the end of the chapter. Finally, some conclusions are drawn.

\subsection{System for Using Decision Support Tools}

The tools are developed as applications. To be able to use the tools, the tool application is connected to a geographical information system (GIS) called C3Fire and a user interface (UI) called SMOKE. SMOKE is used in order to handle the resources and C3Fire is a geographical map-based system for visualizing the resources (Granlund and Johansson, 2003). The systems are connected and communicate according to Figure 16.

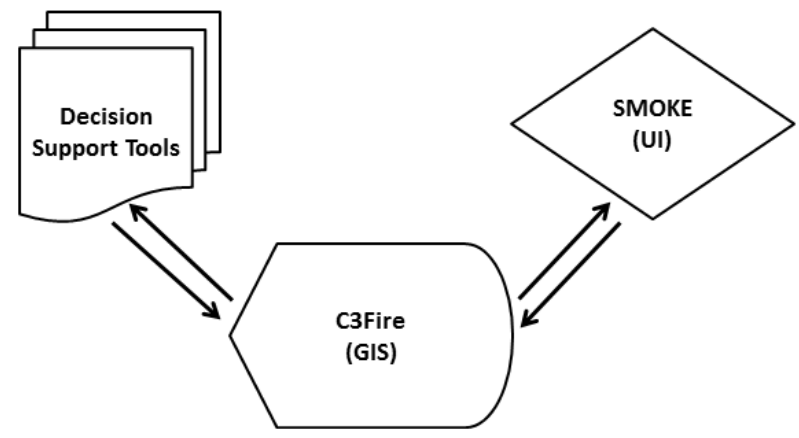

Figure 16. How the tools, the C3Fire and the SMOKE, are connected to each other.

With the GIS application C3Fire, the user obtains an overview of the planning area and of all the resources (fire stations, firefighters, and vehicles) in the planning area. The user controls and manages the resources through the UI SMOKE. Via SMOKE, the user forms crews of firefighters and vehicles and dispatches the resources to accidents and other missions. A picture of the systems is shown in Figure 17. 


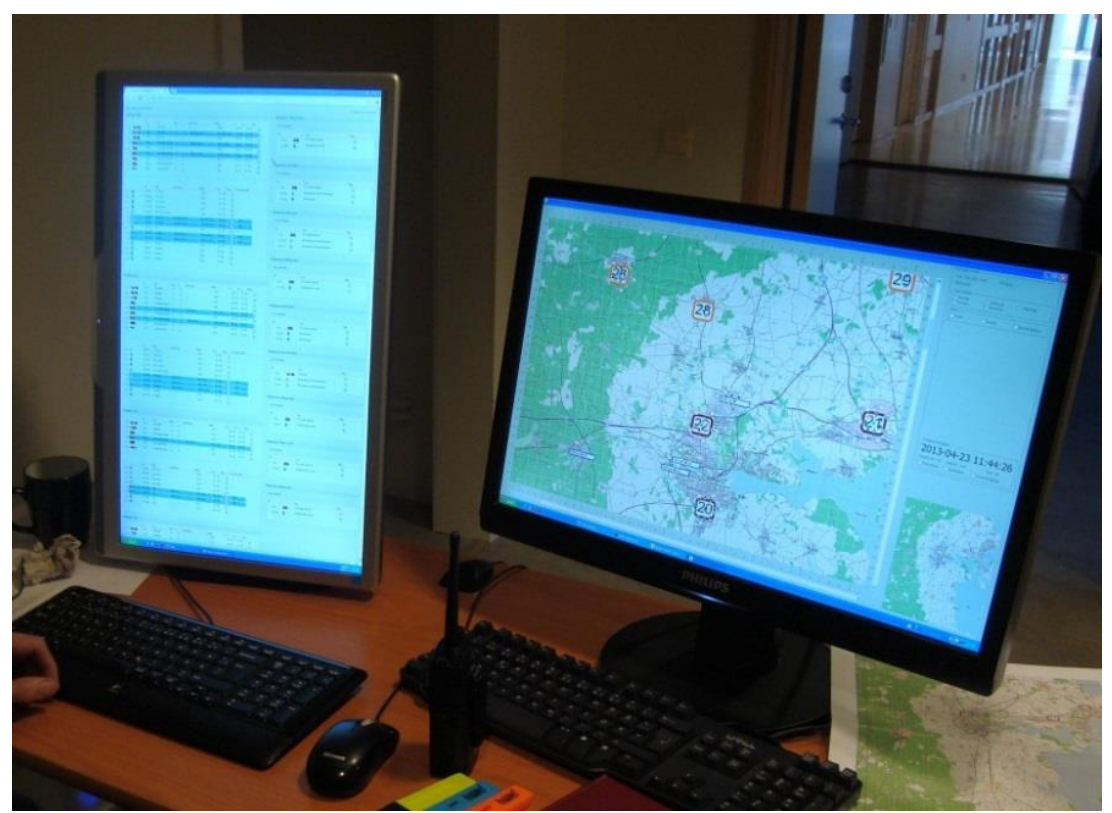

Figure 17. The systems for using the decision support tools. The GIS application (C3Fire) is to the right and the UI (SMOKE) is to the left.

Both in the map (the GIS application) and in the UI, the user has an overview of all the resources, the firefighters' competencies, and resources' locations. An example of the map is shown in Figure 18. The map illustrates all the resources and their current locations. The numbered squares are fire stations. The squares with a red frame are full-time stations, i.e. stations with full-time firefighters, and those with an orange frame are part-time fire stations, i.e. stations with part-time firefighters. The small colored ovals represent firefighters and are colored differently depending on firefighter competencies; see Section 5.4.1. The small numbered rectangles represent vehicles. The numbers represent both to which fire station a vehicle belongs as well as vehicle type. When the resources are not located at the fire stations, there is a brief notation both in the map and in the UI regarding their current mission. The information in the map can be found in a square next to the resource locations. For example, 'Info B23' is a non-urgent information mission for two firefighters. Urgent missions are framed in red. 


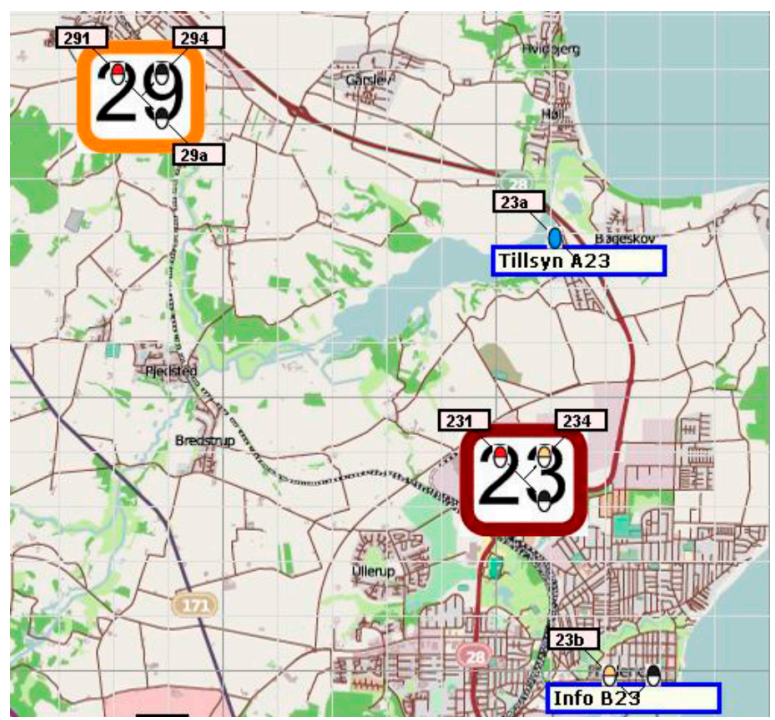

Figure 18. An example of the digital map that illustrates the resources and their current location.

The firefighters and vehicles are handled individually in the system. The user forms crews and provides the vehicle with personnel before they are dispatched to an incident. The user dispatches resources via the UI SMOKE. An example of the UI is shown in Figure 19. The available resources are shown to the left; they are grouped in vehicles and personnel resources. The view to the right shows all the resources that are assigned to various missions. All the resources that are assigned to a specific mission are grouped together. In this example, all the resources are assigned to a traffic accident (Trafikolycka). The available resources (those to the left in Figure 19) are color coded depending on the mission they are assigned to, e.g. they are yellow if they are assigned to an urgent accident, blue-green if they are assigned to a non-urgent mission, and grey if they are located at a break point - a strategic position chosen by the user. Other information that can be found in the UI is the firefighters' competencies, their preparation times, the resources' locations, and whether they are available to respond to an accident. A firefighter is dispatched to an accident by assignment to a vehicle, and then the vehicle is dispatched to the accident site. 


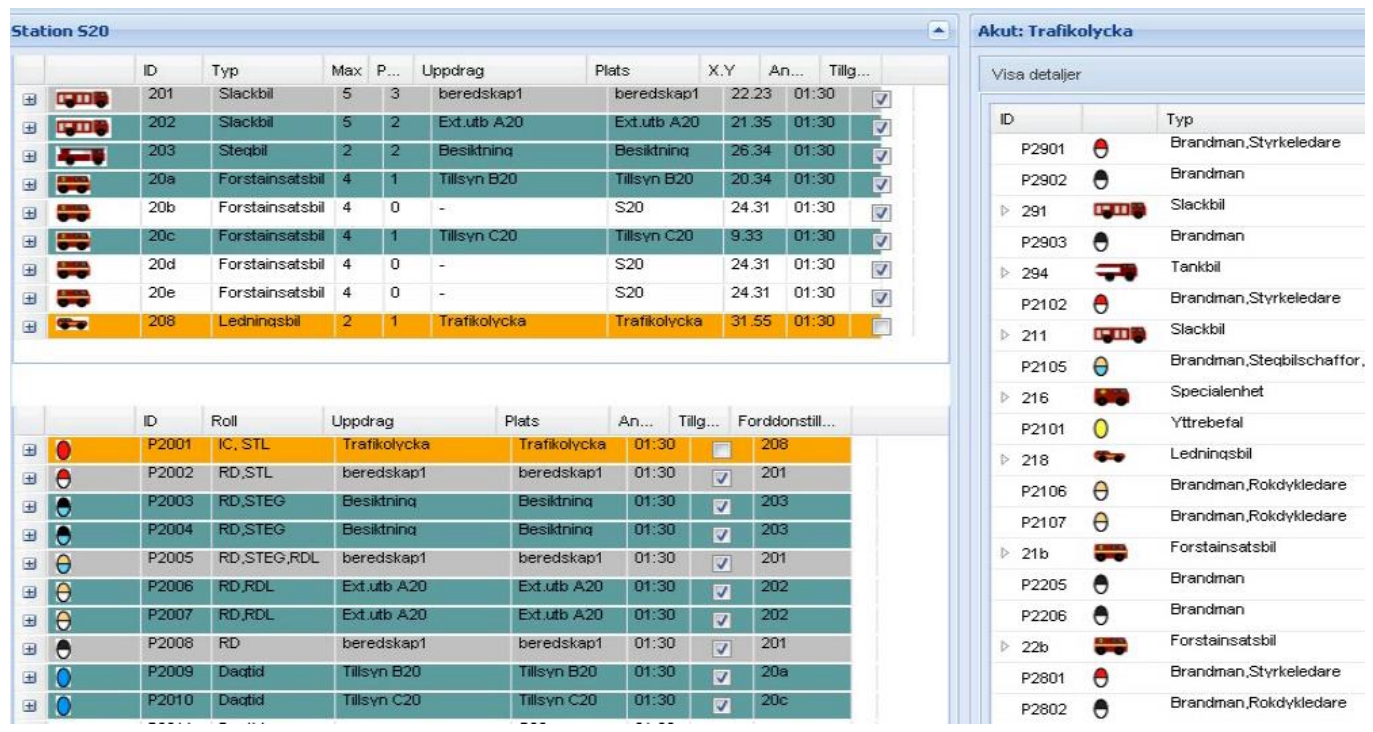

Figure 19. The user interface SMOKE where all the resources are included as well as information about their availability.

\subsection{Tool 1 - Visualization of Preparedness}

The first tool is the preparedness visualizer. Preparedness and how to measure the preparedness is further described in Section 5.1. This tool illustrates the preparedness levels on the map in the system, i.e. via the GIS application.

To illustrate the preparedness, the preparedness value is divided into different intervals as explained in Section 5.5.3. An example of how the preparedness is illustrated is presented in Figure 20. The squares that are red indicate an inadequate preparedness, and those that are green indicate good preparedness. The squares that are orange indicate an acceptable (but somewhat reduced) preparedness. In the system, the preparedness is illustrated for one specific type of accident at a time, and the preparedness can be illustrated for any type of accident. In the test setting it is possible to illustrate the preparedness for traffic accidents, building fires, and fires in high-rise buildings. 


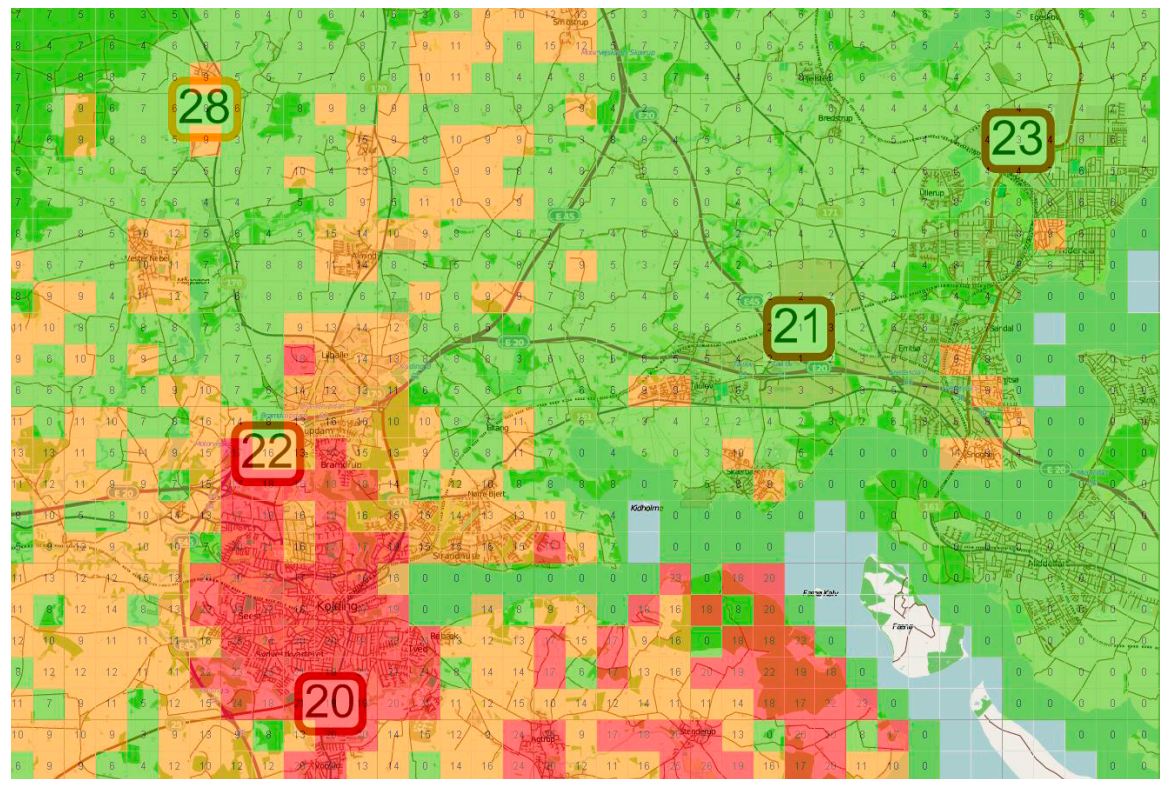

Figure 20. A visualization of preparedness.

The visualization of the preparedness can be used to quickly get an overview of the situation in an area. The overview can support fire and rescue services in decisions regarding which resources to dispatch to accidents (e.g. resources located in green areas) or how resources could be relocated in order to improve the preparedness (e.g. move resources to red areas).

In addition to the preparedness, expected response time as well as the expected risk situation for a particular type of accident can be illustrated. The response time can be illustrated for both first and full responses, i.e. for both when a first resource can reach an accident site as well as when all the required resources for the specific accident type can reach the site.

\subsection{Tool 2 - Dispatching of Resources to Accidents}

The second tool can facilitate decisions about which resources to dispatch to incidents. When the preparedness is calculated for a particular type of accident in a specific position, the resources that contribute to the preparedness are identified. These resources can be illustrated by a mouse click on the map. The user can thus select any position on the map and get the resource information for a chosen accident type. An example for a building fire accident is shown in Figure 21. The resources that contribute to the preparedness (illustrated on the map) are also those that are required for handling the chosen accident type and that have the shortest response time to the selected position. Therefore, this information can be used as a basis for decisions about how resources can be dispatched to accident sites since that usually is the quickest resource/resources (closest) to the accident site. However, there may sometimes be resources that are located closer to the selected position that can respond to the accident site, e.g. first responders and resources that are not required for the particular accident type. The resources are always visible on the map, but they are not suggested for dispatch by the system. 


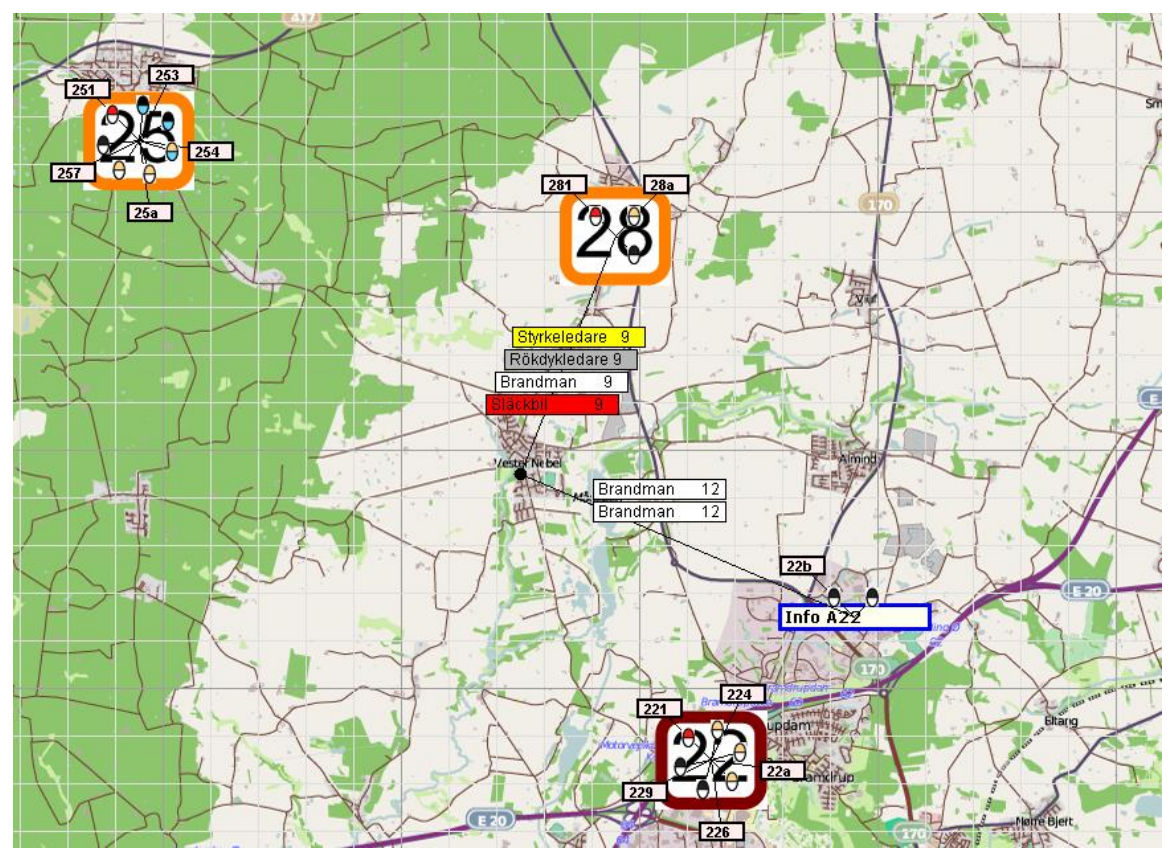

Figure 21. The resources that contribute to the preparedness for building fires.

For the selected position in Figure 21 (the little black dot), the closest resources that are required for building fires are located at fire station 28 and at Info A22. The response time is also depicted for each specific resource. The response time for the resources located at station 28 is nine minutes, and the response time for the resources located at Info A22 is 12 minutes.

Another version of this support tool is available via SMOKE. The user can specify which and how many resources of each type that are desired for an incident at a certain position. Then, the tool gives the user a suggestion for which resources should be dispatched in order to minimize resource response time to a given position.

\subsection{Tool 3 - Relocation of Resources}

The third tools can support the user in decisions regarding how resources can be relocated to improve the preparedness in an area. The tool is based on the relocation model presented in Section 6.1. The tool identifies where the preparedness is inadequate and makes a suggestion as to how resources can be relocated to be closer to areas where is inadequate. The purpose is to improve the preparedness throughout the area, and the preparedness is calculated using the measure presented in Section 5.1.4

A picture on how the tool illustrates suggestions of resource relocations is shown in Figure 22. The picture shows an example of a suggestion where three firefighters and one vehicle are relocated from fire station 26 to a new position (Beredskap_C) and two firefighters and one vehicle from fire station 20 to a new position (Beredskap_B). The red lines in the picture indicate that the resources are recommended for relocation. 


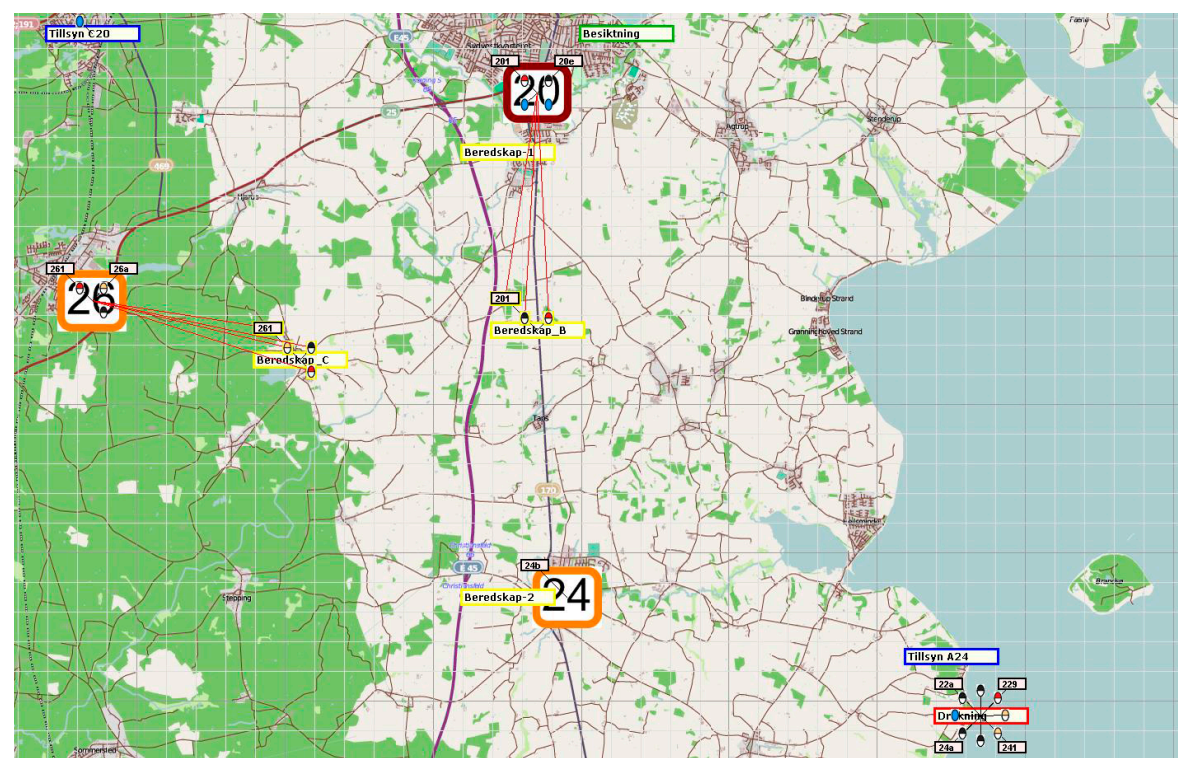

Figure 22. An example of suggestions of how resources can be relocated to improve the preparedness.

The tool focuses on improving the preparedness for areas where the preparedness is considered to be inadequate. Via the tool, the user can visualize the preparedness both before and after any relocation has been made i.e. if the suggested relocations are performed. The user can thereby analyze how the preparedness will change if implementing the suggestions or not. An example is presented in Figure 23. The picture to the left illustrates the preparedness before relocations that are suggested in Figure 22 have been implemented, and the picture to the right illustrates the preparedness after the resources have been relocated. In the picture to the left, there are 50 red squares, i.e. 50 squares with inadequate preparedness, and in the picture to the right there are 34 red squares. In addition, the user can choose to change the suggestions and analyze parts of them or include and analyze their own suggestions if desired.
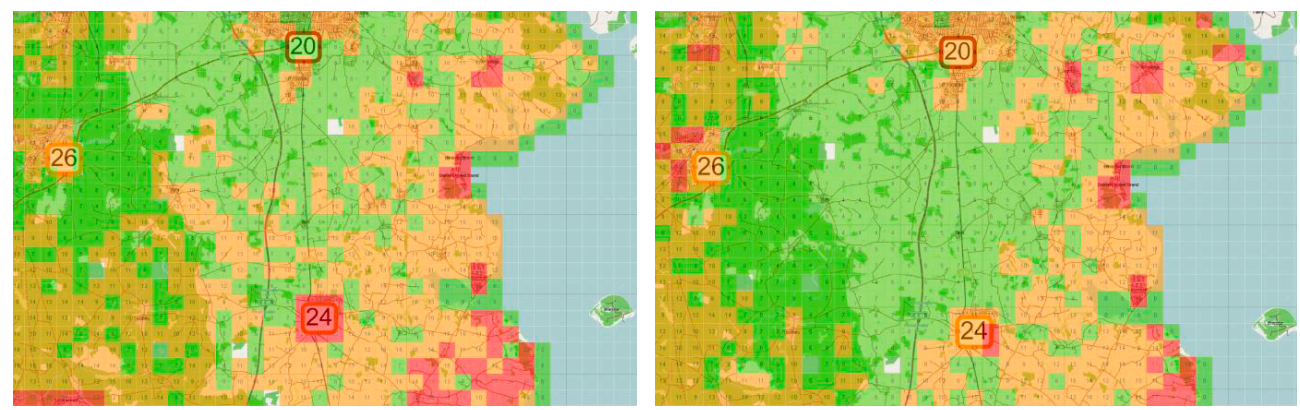

Figure 23. The preparedness before (to the left) and after (to the right) the resources have been relocated according to the produced suggestions in Figure 22. 


\subsection{Validation of the Decisions Support Tools}

To evaluate the three developed decision support tools, two series of experiments have been performed with 13 fire and rescue services in Sweden. In the experiments, fire and rescue service staff had the opportunity to plan and manage resources dynamically. In the first series of experiments, the participants did not have access to the developed tools, while in the second series they had access to the tools.

The experiments were performed in the test setting explained in Section 5.4.1. During the experiment, the participants planned and managed resources with respect to various incidents, both urgent and non-urgent. For example, they dispatched resources to non-urgent missions, such as inspections and training sessions (i.e. preventive work), they dispatched resources to upcoming urgent accidents, and they allocated resources in order to maintain adequate preparedness in the area. Generally, three persons from a fire and rescue service were included in each experiment: one incident commander that made all the decisions, one person that discussed strategies and decisions with the incident commander, and one person that had the role of all included resources in the area, i.e. all the resources on the map. In total, 40 different individuals participated in the experiments.

Before an experiment started, the participants were encouraged to work dynamically with smaller units of resources and to consider the first response time to accidents since that was to be a measure of their performance during the exercise. In the experiments, the users had full flexibility to allocate and dispatch resources in any combination in order to enable dynamic planning.

Each series included seven experiments, and during the experiments many key figures were measured, for example, how well the fire and rescue service staff maintained the preparedness, the resource response times to urgent accidents, and the time it took for the staff to make decisions. The staff also answered some questions as to their experience with the developed tools after the experiment was performed. The questionnaire is presented in Appendix I. The collected information from the experiments has been analyzed in order to compare how well the fire and rescue service (with and without access to the tools) can perform dynamic planning.

\subsubsection{The Impact on Preparedness}

As discussed in Chapter 5, preparedness is a measure that is used to express the ability to take care of new accidents. A good preparedness indicates that resources required for a particular type of accident can quickly respond to the accident site. However, preparedness is subjective, i.e. an individual may believe the preparedness is good in an area while another could consider the preparedness in the same area inadequate. Access to a tool that can illustrate preparedness may facilitate the evaluation process and may help staffs within the fire and rescue service perform a more uniform evaluation of preparedness. The hypothesis was that the participants in the second series of the experiment, with access to the tools, would evaluate the preparedness in a way that would better match the calculated preparedness than participants in the first series of the experiment. To investigate whether the developed tools had any impact on the evaluation process, the participants evaluated the preparedness several 
times during the experiments, i.e. for a number of scenarios. The preparedness for each scenario was evaluated for 13 predefined zones (A-M) by indicating whether the preparedness was good, acceptable, or inadequate in these zones; see Figure 24. The accident types building fires, traffic accidents, and fires in high-rise buildings were considered in the evaluation process. The evaluation was made for all the accident types together, i.e. the total preparedness for the three accident types was evaluated. The figure also illustrates the entire experimental area.

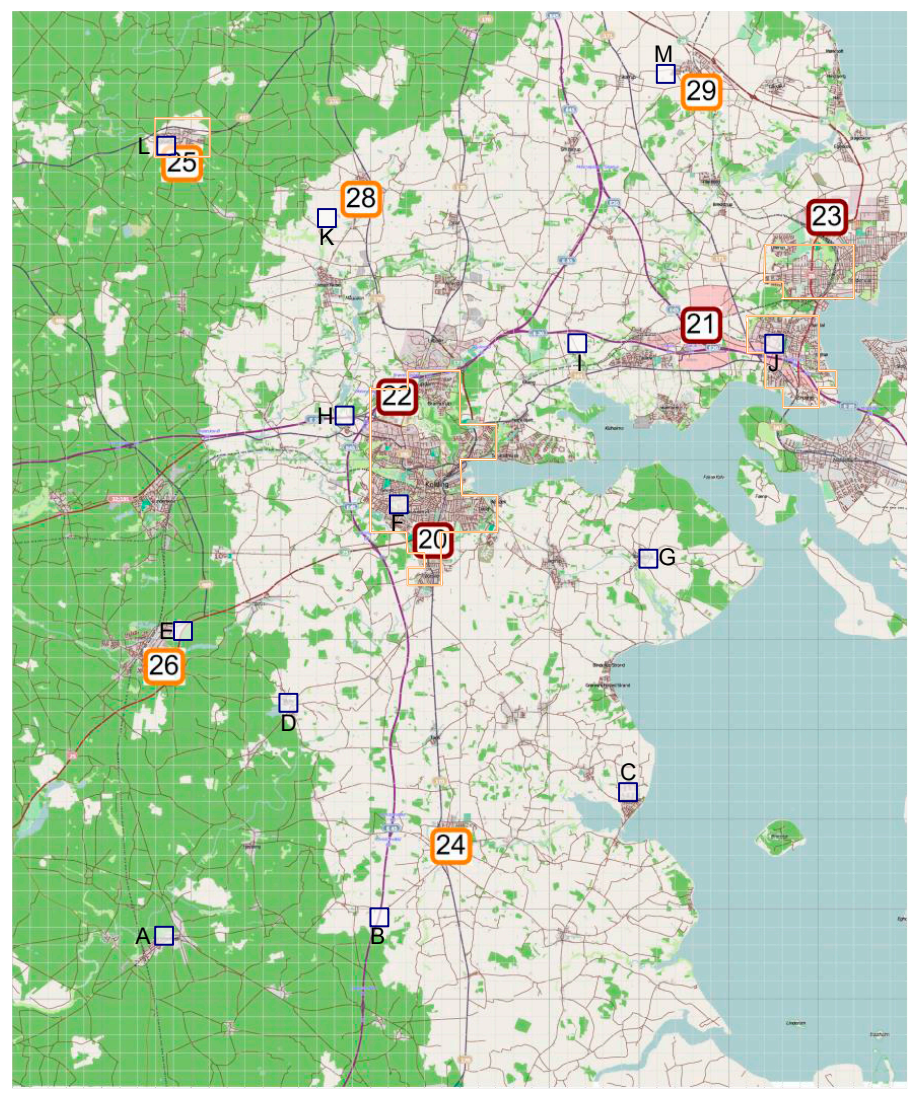

Figure 24. The 13 zones where the preparedness was evaluated for during the experiments.

From the experiments it was demonstrated that the evaluations that matched the calculated preparedness increased from approximately $50 \%$ to just above $54 \%$ in the second series of experiments. However, more telling is that the evaluations that were incorrect in two steps, i.e. when the preparedness was evaluated as inadequate while the calculated value indicated good preparedness, decreased from $4.5 \%$ to $1.5 \%$ in the second series. Also, the standard deviation for the results where the evaluations matched the calculated preparedness value decreased from 11.9 to 7.2 in the second series of experiments. Consequently, this indicates that when the users had access to the developed tools and the preparedness visualization, they also partly used the visualization as a basis to evaluate the preparedness. The users seemed to 
be more consistent in their preparedness evaluations with access to the preparedness visualization.

During the experiments, the time required for the users to evaluate the preparedness was measured both in the first and the second series of experiments. The measured times showed that when the users had access to the tools the evaluation time increased. The evaluation time increased on average from three minutes and 21 seconds in the first series of experiments to four minutes and 57 seconds in the second series of experiments. Thus, the evaluation time increased when the user had access to the tools and various views to illustrate the preparedness for different types of accidents on the map.

One of the most important objectives with the developed tools is that they should be able to support user maintenance of adequate preparedness. Therefore, the preparedness was measured in each experiment for cases when all required resources were dispatched to the current ongoing accidents and when users have had the chance to relocate resources, if possible, to improve the preparedness. This resulted in three such cases per experiment. Three cases were chosen in order to give the users in all the experiments the same opportunities and conditions in terms of both resources and ability to adjust the preparedness. However, the users in the second series of experiments had access to the support tools.

Some indicators of the preparedness were measured for the three cases in all experiments. How the mean values for the indicators differed between the two experimental series is presented in Table 23. A positive value in the table means an improvement in the second series of experiments. The indicators are measured for all zones in the area, i.e. all the zones in the test setting explained in Section 5.4.1, for each case. The measured values for the indicators are the mean value for all zones. That means that the first response time in case 2 was improved (decreased) by $14.55 \%$ on average for all zones in the second series of experiments. The first response time to a zone is the expected time it takes for the nearest resource to reach that particular zone. The column Mean change presents the mean change for the three cases between the two series of experiments, i.e. the first response time was improved (decreased) by one minute and 37 seconds on average in the second series of experiments compared to the first series.

Table 23. Changes in some indicators of the preparedness between the experimental series 1 and 2.

\begin{tabular}{|c|c|c|c|c|c|}
\hline \multirow{2}{*}{\multicolumn{2}{|c|}{ Indicator }} & \multicolumn{4}{|c|}{ Case } \\
\hline & & 1 & 2 & 3 & Mean change \\
\hline \multicolumn{2}{|c|}{ First response time } & $3.03 \%$ & $14.55 \%$ & $8.68 \%$ & $1 \mathrm{~min} 37 \mathrm{sec}$ \\
\hline \multirow{3}{*}{$\begin{array}{l}\text { Mean } \\
\text { response } \\
\text { time }\end{array}$} & Building Fires & $-2.82 \%$ & $11.36 \%$ & $9.02 \%$ & $1 \mathrm{~min} 35 \mathrm{sec}$ \\
\hline & Traffic & $-2.86 \%$ & $11.37 \%$ & $8.94 \%$ & $1 \mathrm{~min} 31 \mathrm{sec}$ \\
\hline & High Building Fires & $1.87 \%$ & $2.36 \%$ & $58.18 \%$ & $6 \mathrm{~min} 46 \mathrm{sec}$ \\
\hline \multicolumn{2}{|c|}{ Preparedness, the worst $10 \%$ zones } & $-4,73 \%$ & $16.79 \%$ & $11.20 \%$ & $8.50 \%$ \\
\hline
\end{tabular}


The expected mean response times for various accident types are based on the resources required for each type of accident according to Table 16 in Chapter 5. The preparation time for firefighters, i.e. the expected time from when an alarm is received until the resources are on the way to the accident site, is included in the response times. For building fires and traffic accidents, the mean response time in case 1 is worse in the second series than in the first series of experiments. This could possibly be due to the unfamiliarity of the users with the support tools at the beginning of the experiment; therefore, they could not make full use of them. For the two other cases, the mean response time is improved in the second series of experiments compared to the first series, and the average mean response time for all three cases is positive. The preparedness for the $10 \%$ of the zones with the worst preparedness was also measured for the three cases. The zones were selected according to the sum of the preparedness for all three accident types. The results for the measured indicators indicate that the developed tools can support user maintenance of adequate preparedness. However, more data and further experiments would be needed to draw more reliable conclusions.

Each time the tool that gives suggestions on how resources can be relocated in order to improve the preparedness were used, the preparedness was measured both before and after the tool were used. The tool was used 27 times, i.e. for 27 different scenarios, in the second series of experiments. The measured preparedness showed that by moving resources as suggested by the tool, the preparedness for the three types of accidents for a scenario can be improved up to $27 \%$. However, the possibilities to improve the preparedness vary significantly between the scenarios. On average, for the 27 scenarios, the preparedness can be improved with almost $9 \%$ if the suggestions are followed.

When the tool was used in the experiments, the users decided to follow the suggestions, partially follow the suggestions, or not follow them as discussed in Section 6.2.4. Since the users could decide whether to use the suggestions, the preparedness was also measured after the users were finished with the resource planning process. If the suggestions were not followed or partly followed, the preparedness was usually not improved by as much as it could have been if the suggestions had been followed as demonstrated by the results from the tool. In Table 24, the average improvement for the 27 scenarios for the same preparedness indicators as just explained above is presented. The first column presents the improvements when resources are relocated exactly as suggested by the tool. The other column presents the final improvements after the users have performed their actual relocations of resources. The indicators are measured for all zones in each of the 27 scenarios, and then an average improvement for all zones for each indicator and scenario are measured. The improvement of the first response time when following the suggestions is $12.27 \%$ on average for all 27 scenarios, which in time corresponds to two minutes and 11 seconds. The first response time is improved for all scenarios if the suggestions are followed. The improvements vary between $1 \%$ and $21 \%$ in the scenarios. However, after the final performed relocations, the first response time increases by $1 \%$ or $2 \%$ in some scenarios. 
Table 24. The average improvements of some indicators of the preparedness when the support tool for how to relocate resources was used in 27 scenarios in the experimental series 2.

\begin{tabular}{llll}
\hline Indicator & \multicolumn{1}{c}{$\begin{array}{c}\text { Improvements when } \\
\text { following the suggestions }\end{array}$} & $\begin{array}{c}\text { Improvements after the } \\
\text { users actual performed } \\
\text { relocations }\end{array}$ \\
\hline \hline First response time & Fire & $12.27 \%-2 \mathrm{~min} 11 \mathrm{sec}$ & $5.91 \%-1 \mathrm{~min} 5 \mathrm{sec}$ \\
$\begin{array}{l}\text { Mean } \\
\text { response } \\
\text { time }\end{array}$ & Traffic & $7.46 \%-1 \mathrm{~min} 39 \mathrm{sec}$ & $5.52 \%-1 \mathrm{~min} 14 \mathrm{sec}$ \\
& High Building Fires & $7.46 \%-1 \mathrm{~min} 39 \mathrm{sec}$ & $5.52 \%-1 \mathrm{~min} 14 \mathrm{sec}$ \\
Preparedness, the worst 10\% zones & $1.12 \%-1 \mathrm{sec}$ & $5.28 \%-2 \mathrm{sec}$ \\
\hline
\end{tabular}

The expected mean response time was improved (decreased) on average for both fire and traffic accidents in all 27 scenarios. For expected fires in high-rise buildings, the mean response times increased in some of the scenarios in both cases. Therefore, the average improvement of the mean response time to fires in high-rise buildings is comparatively low. This probably depend on that resources often were available in cities where there is risk for fires in high-rise buildings, and therefore the preparedness for fires in high-rise buildings was likely already good enough. When improving the preparedness outside cities, resources are moved from cities, and thus the preparedness in cities (for high-rise buildings) might become worse while the preparedness for traffic and fire accidents will be improved. The preparedness for the $10 \%$ of the zones with the worst preparedness can be improved by up to $32 \%$ if the suggestions produced by the tool are followed, on average for the 27 scenarios by almost $13 \%$.

In the experiments, the users followed or partly followed the suggestions in $70 \%$ of the cases. To partly follow a suggestion means that the user, for example, relocates resources other than those suggested, relocates more or fewer resources than suggested, or relocates resources to other locations than suggested. By exactly following the suggestions, the results illustrate that the preparedness can be improved. They also show that the preparedness can be improved using the suggestions as support for how to relocate resources, as the users did in most of the cases; however, usually not as much as when the suggestions are followed. It should also be noted that the ability to improve the preparedness varies for the scenarios as they have different conditions.

\subsubsection{Importance for Dispatching Resources}

During the experiments, a number of accidents occur and the users should dispatch resources to these accidents. To investigate whether access to the support tools could contribute to a more efficient dispatching of resources, the response times to the accidents were measured for the two series of experiments. The first response time, i.e. the time from when an alarm was received until the first responding resource arrived at the accident site, and the processing time for an alarm, i.e. the time from when an alarm was received until the first responding resource was on the way to the accident site, was measured. The average first response times 
and the average processing time for an alarm for the two experimental series are presented in Table 25 .

Table 25. Average processing time for an alarm and average first response time for the two experimental series.

\begin{tabular}{lll}
\hline Time & Average time in series 1 & Average time in series 2 \\
\hline \hline Processing time for an alarm & $1 \mathrm{~min} 59 \mathrm{sec}$ & $2 \mathrm{~min} 6 \mathrm{sec}$ \\
First response time & $14 \mathrm{~min} 24 \mathrm{sec}$ & $14 \mathrm{~min} 48 \mathrm{sec}$ \\
& & \\
\hline
\end{tabular}

As the table reveals, the difference is negligible between the average times in the two series. For both the processing time for an alarm and the first response time, the average times are slightly longer in the second series. However, the measured times vary significantly between each other, especially the first response times. In each experiment, the two times were measured for four accidents. Since each series consisted of seven experiments, the average values in Table 25 include 28 measured values. To be able to draw any further conclusions regarding whether access to the tools could contribute to a more efficient dispatch of resources, more data would be required. The present results do not really indicate whether the tools support or prevent a quicker dispatching of resources.

\subsubsection{The Participant Comments}

When an experiment was completed, the participants answered a few questions about the experience of dynamic planning and how they experienced the experiments. The questionnaire is presented in Appendix I. One question was about the participant experience in using smaller units and working with dynamic planning. The participants answered by entering a value on a seven-point scale from infrequently to frequently. Of the 40 participants, 22 chose a value from 1 to 3 and 18 chose a value from 4 to 7 . It was a good mix of the participant experience of dynamic planning; there were a few more participants that did not have so much experience. Accordingly, the experiments also contributed to sharing knowledge regarding dynamic planning among fire and rescue services in Sweden. In general, the participants thought that they had enough support from the system in both the first and second series of experiments, even if they did not have access to the developed support tools in the first series. This is probably because the fire and rescue services currently do not have any support tools like these presented in this chapter that can support them in recourse planning. The participants in the first series were therefore satisfied with only access to the map application and the view of all the resources in the UI. From the answers to the questions, it was also found that the majority of the participants felt that the experiments were realistic, both in terms of the exercise in general and regarding the accidents that occurred during the experiments.

The participants in the second series also answered the questions:

1. How did you experience the decision support tools you had the opportunity to try in the experiment? 
2. Besides the quality and the design of the tools you tested during the experiment, do you think this kind of support is helpful in planning situations for the fire and rescue service?

The questions were answered on a five-point scale; the first question ratings were from unsatisfactory to satisfactory and the second question ratings were from unhelpful to very helpful. The mean value of the answers to the first question was 3.44 and to the second question was 4.44 . This indicates that the participants that tested the developed tools believed that such support the tools can provide assistance in the resource planning process for the fire and rescue service.

Other issues that developed from the evaluations of the experiments were that the system and the tools could be useful for training exercises for fire and rescue service staff. Many participants indicated that they found the tools useful and that they could use them to get a good overview of the situation in the area, while others found them a bit stiff and lacking in certain functions. For example, functions lending the ability to see the resource expected arrival times to accidents, to zoom in the map application, and to more easily manage and dispatch the resources in the map application, which now must be done via the user interface. The ability to illustrate preparedness was highlighted as good and useful, but some of the participants believed that the tools took rural areas into too much consideration. Since firefighters usually are located at stations in cities and suburbs, the users thought it is was quite unfamiliar when the system sometimes suggested locating resources on the countryside. To get suggestions regarding how resources could be dispatched to accidents and how they could be relocated to improve the preparedness were in general positive and useful, especially since this gave the users the possibility to compare their own ideas with the systems.

\subsection{Conclusions}

The performed experiments with fire and rescue services in Sweden show that the developed tools can support the resource planning process. For example, the users that had access to the tools were able to maintain a better preparedness than the users who did not have access to the tools. However, the planning time seems to increase when using the tools, probably because it takes some extra time to use them. This could also depend on that the users were quite unfamiliar with the system and the tools. When users become more experienced, the planning time would probably be improved compared to the performed experiments results.

With the ability to illustrate the preparedness, such a tool could help engender a more uniform perception of the term preparedness. This may facilitate communication between firefighters as well as between fire and rescue services and other organizations. It would likely make it easier if individuals have the same interpretation.

By only considering the quantitative results from the experiments, it is not possible to draw any definite conclusions about whether access to the developed tools contributes to better dynamic planning. However, the results from the questions the participants answered after each experiment and other comments from the participants indicate that the developed tools were useful and that such support would be helpful in their daily work. 
An interesting observation was made that the participants often used the tools to customize their plans to how they usually plan. They often partly followed the tools' suggestions about how to dispatch and relocate resources. For example, the participants often relocated resources to a fire station close to the suggested position instead of the suggested position. In addition, at times they also relocated or dispatched other, fewer, or additional resources than those that were suggested. The tools could then be adapted to the users' actions. However, since limitations deteriorate the theoretical benefit of the suggestions this may be counterproductive. By not including any limitations in the suggestions to which locations resources can be relocated to, or how resources can be dispatched, the user must decide whether any changes of the tool suggestions should be made and evaluate how that would affect the preparedness.

Even though the participant experience of the developed tools, in general, seems to be positive, there are improvements that can be made. For example, the system and applications could be more user-friendly and the performance of the tools could be improved. A challenge is to decrease the time it takes to use the tools and to obtain a good view of the situation with the support of the system. There are certainly other tools that could be helpful for the fire and rescue service, for example, support for how to prioritize urgent and non-urgent accidents and missions, or making it possible to follow the resources on the map on the way to their destination and simultaneously display the resources' remaining response times when desired.

The system could also be used for different types of exercises and training sessions for the fire and rescue service. It is possible to develop any type of scenario, e.g. any geographical area can be used in the system, and new types of resources can be inserted and any desired types of accidents or missions can occur in the area. That means, the fire and rescue service can perform exercises in different types of environments and management structures. 


\section{Conclusions and Discussion}

In this thesis, operations research (OR) methodology has been used in order to develop decision support tools for the fire and rescue service so they can efficiently manage, coordinate and dispatch fire and rescue resources with respect to the present demand for service.

Most of the previous OR research that has been performed within the fire and rescue service area support a strategic and a static fire and rescue service planning. The reason might be that the fire and rescue service has been traditionally characterized by relative static planning and resource management. One of the most studied planning challenges has been to determine where fire stations should be located or how many fire stations there should be in an area.

However, the conducted interviews made with fire and rescue services as well as the increasing amount of research concerning dynamic and operational planning indicate that a new way of approaching the management of fire and rescue resources is gaining ground. Many of the interviewed fire and rescue services stated that the fire and rescue services in Sweden have started to deploy smaller units instead of only relying on the traditional units with five firefighters. Some of the reasons are to be able to better respond to incidents, to be able to use the smaller units for preventive work as well as maintaining adequate first response coverage.

Using smaller units that cannot be expected to be found at fire stations when there is an alarm gives rise to increasing complexity in the resource planning process. It is, for example, no longer obvious which resources should be dispatched to an incident or where resources should be located to best be available to respond to incidents. It becomes important to keep track of the units as well as the firefighters' individual competences.

The decision support tools developed in this thesis have, through tests and experiments, showed that they can support the fire and rescue service in the planning process.

An optimization model for the problem of strategically locating fire and rescue resources has been developed. The model locates resources in order to minimize the response times for both the first responding resource as well as the last responding resource with respect to different types of accidents. By using the model, it is shown that the response time to housing fires and traffic accidents can be reduced by around $20 \%$. Also, a significantly larger proportion of the accidents can be reached within 10 minutes according to the proposed solutions. The developed location model has been solved for Östergötland County in Sweden. The produced solutions show that the resources are located slightly closer to high-risk areas for traffic accidents. This may be due to that the traffic accidents outnumber the fire accidents in the input data by a factor 2.6. In Sweden, it general occur more traffic accidents than building fire accidents. The traffic accidents represented $19 \%$ of all the rescue service efforts made in 2012, and the building fires $11 \%$ (MSB, 2013). It is, however, just as important to quickly reach a fire accident as it is to reach a traffic accident. Since fire accidents in general also are costlier than traffic accidents, it would be interesting to also weight the response times for different types of accidents in order to find more balanced solutions regarding the different 
types of accidents. It would also be interesting to solve the model for areas with other variations in the demand.

To support the more complex operational planning of fire and rescue resources, three decision support tools based on two optimization models have been developed. The first developed tool can help illustrate the preparedness, the second to select appropriate vehicles and firefighters to dispatch to an accident, and the third to suggest how resources can be relocated to maintain an adequate preparedness for new accidents. The tools are developed in order to help the fire and rescue service to always maintain an adequate level of preparedness for handling accidents. To evaluate the preparedness, a quantitative measure has been defined and serves as basis to the tools.

The three support tools have been evaluated through experiments performed with various fire and rescue services in Sweden. Results from the experiments indicate that the developed tools can support the fire and rescue service in coordinating and dispatching resources as well as maintaining an adequate preparedness to accidents. The users that had access to the tools were able to maintain a better preparedness than the users who did not have access. However, the planning time seems to increase when using the tools since it take some extra time to use them. This may also partly depend on the users' unfamiliarity with the system and the tools and will probably slightly decrease when the users become more experienced.

The results from the experiments also indicate that fire and rescue service personnel, even very close colleagues, perceive preparedness differently and sometimes as a fuzzy concept. With the ability to illustrate the preparedness, it was found that the difference was reduced. With access to the tools, the participants' got a more uniform perception of preparedness. A preparedness measure as the one developed in this thesis might likely help to reduce the fuzziness and contribute towards a standardization of the perception of preparedness.

By considering the quantitative results from the experiments, it is not possible to draw definite conclusions whether the tools contribute to better dynamic planning. Further tests and experiments would be needed to draw more reliable conclusions. More data need to be collected to better be able to analyze the tools and their importance for the planning process. The tools must also be tested by a larger number of fire and rescue services to get a better basis of various fire and rescue service organizations. However, the participants' experiences of the tools were in general positive and their comments indicated that the tools were useful and that they could support their daily work.

Interesting observations from the experiments also included that the participants often customized their decision making to traditional planning, e.g. they often located resources at fire stations instead of the positions suggested by the support tools. In general, the participants only partly followed the suggestions produced by the tools regarding planning and managing resources. However, at times they did use suggestions they considered unreasonable and sometimes they did not use suggestions they considered reasonable. A challenge is then that the perception of how resources should be managed and planned better must correlate between the tools and the emergency services staff. The system can also be more user-friendly with better maps and better user interface. The system and the support could certainly also be 
improved by displaying resources' travel times to incidents or by displaying the resources' movements on the map. Another challenge is to reduce the time it takes to use the tools. The performance of the tools as well as the developed optimization models that serve as basis for the tools must then be improved. There might also be improved solutions methods that can be used to provide quicker, and maybe better, solutions to the models.

Optimization based support tools could also be used for various tests, exercises and training sessions. Fire and rescue services can for example use the tools to perform exercises in different types of environments and management structures, any types of resources can be used, and it might be possible to practice planning and management for all types of situations, both small and large incidents. This makes it possible for fire and rescue services to perform tests and exercises in resource planning in their own areas according to their own organization.

There are also other types of tools that could be developed in order to support the fire and rescue service in the planning process. For example, to support decisions regarding if resources that are dispatched to an incident, first need to be rerouted before they can continue to the incident. If, for example, a ladder vehicle operator has been dispatched to an accident, but currently are assigned to a small unit to perform a non-urgent mission, it may be necessary to first route the operator to the fire station to pick up the ladder vehicle, before proceeding to the accident. Supporting the fire and rescue service in making decisions about how to prioritize incidents, e.g. urgent and non-urgent missions, might also be valuable. Another interesting research issue is how fire and rescue resources should be used for preventative work. It might be possible to conduct this planning with more respect to the level of preparedness. Also, how non-traditional response resources can be more utilized in fire and rescue service responses is an interesting research question. For example, how home care nurses or security guards can be used as first responders to support the fire and rescue service in various situations. More research is, thus, needed concerning planning of fire and rescue resources to facilitate decision making in the planning process, both for operational planning, but also for strategic and tactical planning to adapt the fire and rescue service operation to the new working approach when resources are no longer assumed to work in groups of four and wait for an alarm at a fire station. 


\section{References}

AFS 2007:7, Arbetsmiljöverkets författningssamling, Rök- och kemdykning. Arbetsmiljöverket Publikationsservice, ISBN 978-91-7930-484-2.

http://www.av.se/dokument/afs/afs2007_07.pdf (2014-04-01)

Altay, N., Green, W. G. (2006) OR/MS research in disaster operations management. European Journal of Operational Research, 175, 475-493.

Andersson Granberg, T. (2013) Preparedness Measures for Emergency and Disaster Response, In: Zeimpekis V., Ichoua S., Minis I., (Eds.), Humanitarian and Relief Logistics: Research Issues, Case Studies and Future Trends, pp. 59-75. Springer-Verlag New York.

Andersson, T., Lundgren, J., Wellving, A. Axelsson, A. (2005) OPERA - Optimerad och effektiv resursanvändning vid räddningstjänst, En förstudie. Linköpings Universitet, Räddningsverket.

Andersson, T., Särdqvist, S. (2007) Planning for effective use of Fire and Rescue Service Resources, Proc. of Interflam, London, UK, 1561 - 1566.

Andersson, T., Värbrand, P. (2007) Decision support tool for ambulance dispatch and relocation. Journal of the Operational Research Society, 58, 195-201.

Badri, M. A, Mortagy, A. K., Alsayed, C. A. (1998) A multi-objective model for locating fire stations. European Journal of Operational Research, 110, 243-260.

Batta, R., Mannur, N. R. (1990) Covering-Location Models for Emergency Situations That Require Multiple Response Units. Management Science, Vol. 36, No. 1, 16-23.

Beraldi, P., Bruni, M. E. (2009) A probabilistic model applied to emergency service vehicle location. European Journal of Operational Research, 196, 323-331.

Berman, O., Verter, V., Kara, B. K. (2005) Designing emergency response networks for hazardous materials transportation. Computer \& Operations Research, Vol. 34, No. 5, 13741388.

Bertsimas, D., Tsitsiklis, J.N. (1997) Introduction to Linear Optimization. Athena Scientific, Dynamic Ideas, USA, Fourth printing, ISBN 978-1-886529-19-9.

Bishop, R. L., Peterson, G. L., Berlin, G. N. (1971) Towards a Methodology for Evaluation of Fire Protection Systems in Appalachia, Socio-Econ. Plan. Sci., Vol. 5, 145-158.

Björnberg, B., Melin, G. (2003) Att beställa och utforma räddningsinsatser med Jönköping som exempel. Elanders Skogs Grafiska, Räddningsverket, ISBN 91-7253-210-6.

Brotcorne, L., Laporte, G., Semet, F. (2003) Ambulance location and relocation models. European Journal of Operational Research, 147 451-463. 
Burke, E.K., Kendall, G. (2005) Search Methodologies; Introductory Tutorials in Optimization and Decision Support Techniques. Springer Science+Business Media, Inc, IBSN-10: 0-387-23460-8.

Carter, M. C., Chaiken, J. M., Ignall, E. (1972) Response Areas for Two Emergency Units. Operations Research, Vol. 20, No. 3, 571-594.

Chevalier, P., Thomas, I., Geraets, D., Goetghebeur, E., Janssens, O., Peeters, D., Plastria, F. (2012) Locating fire stations: An integrated approach for Belgium, Socio-Economic Planning Sciences, 46, 173-182.

Church R., ReVelle C. (1974) The maximal covering location problem, Papers in Regional Science, 32(1), 101-120.

Church, R., Sorensen, P., Corrigan, W. (2001) Manpower Deployment in Emergency Services. Fire Technology, 37, 219-234.

Coskun, N., Erol, R. (2010) An Optimization Model for Locating and Sizing Emergency Medical Service Stations. J Med Syst., 34:43-49.

Dahlgren, A., Harrie, L., Axelsson, A. (2009) Planning Rescue Services with Non-Stationary Rescue Units. Fire Technology, 45, 239-255.

Departementsserien DS 2009:47, Reformen Skydd mot olyckor - en uppföljning med förslag till utveckling. Stockholm Allmänna förl. ISBN 978-91-38-23288-0.

http://www.regeringen.se/content/1/c6/13/31/43/65a96c17.pdf (2013-08-13)

Dimopoulou, M., Giannikos, I. (2004) Towards an integrated framework for forest fire control. European Journal of Operational Research, 152 476-486.

Erkut, E., Bozkaya B. (1999) Analysis of aggregation errors for the p-median problem, Computers \& Operations Research, 26, 10-11, 1075-1096.

Geroliminis, N., Karlaftis, M. G., Skabardonis, A. (2009.) A spatial queuing model for the emergency vehicle districting and location problem. Transportation Research Part B, 43, 798-811.

Goldberg, J. B. (2004) Operations research Models for the Deployment of Emergency Service Vehicles. EMS Management Journal, Vol. 1, No. 1, 20-39.

Granlund, R., Johansson, B. (2003) Monitoring Distributed Collaboration in C3Fire Microworld, in S.G. Schiflett, L.R. Elliott, E. Salas and M.D. Coovert, (Eds.), Scaled Worlds: Development, Validation and Applications, Aldershot, Ashgate.

Haghani, A., Yang, S. (2007) Real-Time Emergency Response Fleet Deployment: Concepts, Systems, Simulation \& Case Studies, in Zeimpekis, V., Tarantilis, C. D., Giaglis, G. M., Minis, I., (Eds.), Dynamic Fleet Management, pp. 133-162. New York: Springer. 
Hakimi, S. L. (1965) Optimum distribution of switching centers in a communication network and some related graph theoretic problems. Operations Research, 13, 462-475.

Halpern, J. (1979) Fire Loss Reduction: Fire Detectors vs. Fire Stations. The institute of Management Sciences, Vol. 25, No 11, 1082-1092.

Halpern, J., Sarisamlis, E., Wand, Y. (1982) An Activity Network Approach for the Analysis of Manning Policies in Firefighting Operations. Management Science, Vol. 28, No. 10, 11211136.

Hansen P., Mladenović N. (2001) Variable Neighborhood Search: Principles and applications, European Journal of Operational Research, 130, 449-467.

Hansen P., Mladenović N., Perez-Brito D. (2001) Variable Neighborhood Decomposition Search, Journal of Heuristics, 7, 335-350.

Hogg, J. M. (1968) The Siting of Fire Stations. Operational Research Society, Vol. 19, 275287.

Ignall, E., Carter, G., Rider, K. (1982) An Algorithm for the Initial Dispatch of Fire Companies. Management Science, Vol. 28, No 4, 366-378.

Ingolfsson, A., Erkut, E., Budge, S. (2003) Simulation of single start station for Edmonton. EMS Journal of the Operational Research Society, 54, 736-746.

Kolesar, P., Blum, E. H. (1973) Square Root Laws For Fires Engine Response Distance. Management Science, Vol. 19, No. 12, Application Series, 1368-1378.

Kolesar, P., Walker, W. E. (1974) An Algorithm for the Dynamic Relocation of Fire Companies. Operation Research, Vol. 22, No. 2, 249-274.

Kothari, C. R. (2004) Research Methodology - Methods and Techniques. New Age International (P) Ltd., Publisher, Second Edition, ISBN 81-224-1522-9.

Larsen, A., Madsen, O. B. G., Solomon, M. M. (2007) Classification of dynamic vehicle routing systems, in Zeimpekis, V., Tarantilis, C. D., Giaglis, G. M., Minis, I., (Eds.), Dynamic Fleet Management, pp. 19-40. New York: Springer.

Larson, C. (2004) Decision Models for Emergency Response Planning. Kamien, David G., (Eds.), The McGraw Hill Handbook of Homeland Security, The McGraw-Hill Companies, Inc., Chapter 57, pp. 911-924, ISBN 007-144665-6.

Leyva, J. A. O. (2011) A Microscopic Traffic Simulation Based Decision Support System for Real-Time Fleet Management. Thesis Presented to Obtain the Qualification of Doctor from the Universitat Politècnica de Catalunya.

LSO 2003:778, Lag om skydd mot olyckor http://www.riksdagen.se/sv/Dokument-Lagar/Lagar/Svenskforfattningssamling/Lag-2003778om-skydd-mot-ol_sfs-2003-778/ (2013-02-25) 
Lundberg, J., Granlund, R., Fredäng, A. (2012) Scenario play workshops - Co-design of emergency response scenarios for information technology design in collaboration with emergency response personnel. Proceedings of the $9^{\text {th }}$ International ISCRAM Conference Vancouver, Canada.

Lundgren, J., Rönnqvist, M., Värbrand, P. (2010) Optimization. Studentlitteratur AB, Lund, Edition 1:1, ISBN 978-91-44-05308-0.

Marianov, V., ReVelle, C. (1992) The Capacitated Standard Response Fire Protection Siting Problem: Deterministic and Probabilistic Models. Annals of Operations Research, 40, 303302.

Martinsson, B. (2013) Varifrån kommer vi...och vart har vi kommit? En sammanställning över räddningstjänstens i Jönköpings kommun insatsstatistik under perioden 1996 - 2012. Local Report, Räddningstjänsten Jönköping, Version 2013-08-15.

Mattsson, B., Juås, B. (1997) The importance of the timefactor in fire and rescue service operation in Sweden. Accid. Anal. And Prev., Vol. 29, No. 6, 849-857.

Michalewicz, Z., Fogel, D.B. (2004) How to Solve It: Modern Heuristics. Springer-Verlag Berlin Heidelberg, ISBN 3-540-22494-7.

Myndigheten för Samhällsskydd och Beredskap (MSB), (2011) Statistik och Analys, Räddningstjänst i siffror 2010. Publ.nr MSB275 - juli 2011, ISBN 978-91-7383-143-7.

Myndigheten för Samhällsskydd och Beredskap (MSB), (2013) Statistik och analys, Räddningstjänst i Siffror 2012. Publ.nr MSB560 - juni 2013, ISBN 978-91-7383-342-4.

$\mathrm{Ng}$, K. L., Raff, P. (2007) A generalization of the firefighter problem on Z X Z. Discrete Applied Mathematics, Vol. 156, No. 5, 730-745.

Norrköpings Brandförsvar. (2008) Utredning om brandstationer i Norrköping, Lokalisering och bemanning, Local report, The municipality of Norrköping.

Persson, A. (2006) Sjuksköterskor som förstainsatspersoner vid kommunal räddningstjänst Är sambruk samhällsekonomiskt lönsamt? C-uppsats 2006/04/18, Avdelningen för Nationalekonomi och statistik, Karlstad University.

http://www.diva-portal.org/smash/get/diva2:6149/FULLTEXT01.pdf (2013-08-13)

Resende, M.G.C. and Silva, R.M.A. (2009) GRASP: Greedy randomized adaptive search procedures. Encyclopedia of Operations Research and Management Science, Cochran, J.J. Cox, L.A. Keskinocak, Jr., P. Kharoufeh, J.P. and Smith J.C., (Eds.), vol. 3, pp. 2118-2128Wiley, 2011.

ReVelle, C., Snyder, S. (1995) Integrated Fire and Ambulance Siting: A Deterministic Model. Socio-Econ. Plann. Sci., Vol. 29, No. 4, 261-271.

Rosenberg, T. (2001) Räddningstjänst i förändring - ledarskap och metodik för säkerhet och skydd mot olyckor, Tommy Rosenberg och Räddningsverket, ISBN 91-7253-093-6. 
Räddningsverket (2008) Räddningstjänst i samverkan - Ansvar och uppgifter för räddningstjänstansvariga myndigheter och samverkande organisationer. Räddningsverket och medverkande organisationer, 2: a upplagan, ISBN 978-91-7253-342-4.

Schilling, D., Elzinga, D. J., Cohon, J., Church, R., ReVelle, C. (1979) The Team/Fleet Models for Simultaneous Facility and Equipment Siting. Transportation Science, Vol. 13, No. 2.

Schilling, D.A., ReVelle, C., Cohon, J., Elzinga, D. J. (1980) Some models for fire protection locational decisions. European Journal of Operational Research, 5, 1-7.

Schmid, V., Doerner, K. F. (2010) Ambulance location and relocation problems with timedependent travel times. European Journal of Operational Research, 207 1293-1303.

Schreuder, J. A. M. (1981) Application of a location model to fire stations in Rotterdam. European Journal of Operational Research, 6, 212-219.

Silva, F., Serra, D. (2008) Locating emergency services with different priorities: the priority queuing covering locating problem. Journal of the Operational Research Society, 59, 12291238.

Simpson, N. C., Hancock, P. G. (2009) Fifty years of operational research and emergency response. Journal of the Operational Research Society, 60, S126-S139.

SOAF (2012), Svenska Operationsanalysföreningen, http://www.soaf.se/index.php/vad-aer-oa (2012-09-06).

Svensson, S., Cedergårdh, E., Mårtensson, O., Winnberg, T. (2005) Taktik, ledning, ledarskap. Myndigheten för samhällsskydd och beredskap. Tryck AB Danagårds Grafiska, Andra tryckningen. ISBN 978-91-7383-005-8.

Sveriges Kommuner och Landsting, http://www.skl.se/kommuner_och_landsting (2013-0813)

Swersey, A.J. (1982) A Markovian Decision Model for Deciding How Many Fire Companies to Dispatch. Management Science, Vol. 28, No. 4, 352-365.

Swersey, A.J. (1994) The Deployment of Police, Fire, and Emergency Medical Units. Pollock, S. M., Rothkopf, M. H., Barnett A., (Eds.), Handbooks in OR \& MS, Vol. 6, Elsevier Science B.V., pp. 151-200.

Toregas, C., Swain, R., Re Velle, C., Bergman, L. (1971) The Location of Emergency Service Facilities. Operations Research, Vol. 19, No. 6, 1363-1373.

Weinholt, Å., Andersson Granberg, T. (2013) En samhällsekonomisk utvärdering av samarbete mellan räddningstjänst och väktare. Arbetsrapport $\mathrm{Nr}$ 6, Linköping University, CARER Rapport Nr. 5, URL 1960:729. 
Yang, B., Viswanathan, K., Lertworawanich, P., Kumar, S. (2004) Fire Station Districting Usin Simulation: Case Study in Centre Region, Pennsylvania, Journal of Urban Planning and Development, Vol. 130, No. 3, 117-124.

Yang, L., Jones, B. F., Yang, S-H. (2007) A fuzzy multi-objective programming for optimization of fire station locations through genetic algorithms. European Journal of Operational Research, 181, 903-915.

Zaki, A. S., Cheng, H. K. (1997) A Simulation Model for Analysis and Management of An Emergency Service System. Socio-Econ. Plann. Sci., Vol. 31, No. 3, 173-189.

\section{Interviews}

Olofsson, M., Myndigheten för Samhällsskydd och Beredskap, 2013-12-10

Räddningstjänsten Jönköping, Interview in Jönköping, 2011-04-29

Räddningstjänsten Kinda Kommun, Interview in Kisa, 2011-02-16

Räddningstjänsten Storgöteborg, Interview in Göteborg, 2011-08-10

Räddningstjänsten Syd, Interview in Lund, 2011-05-20

Räddningstjänsten Östa Götaland 1, Interview in Linköping, 2011-02-07.

Räddningstjänsten Östa Götaland 2, Interview in Linköping, 2011-03-25.

Räddningstjänsten Östa Götaland 3, Interview in Norrköping, 2011-03-15.

SOS Räddningscentral Norrköping, Interview in Norrköping, 2011-03-25

Södertörns Brandförsvarförbund, Interview in Stockholm, 2011-03-04 


\section{Appendix I - Questionnaire}

The questionnaire that was filled out by the participants after each experiment. The average value (for all participants, from both experimental series) for each question is marked.

1. Jag anser att områdets likhet med det område jag själv verkar i är:

$$
\text { olikt 1---2---3--- }
$$

2. Min erfarenhet av att arbeta med uppdelade styrkor / dynamisk planering är:

$$
\text { obefintlig 1---2------------7 vardaglig }
$$

3. De hjälpmedel vi hade för att få översikt över beredskap för brand i byggnad var:

$$
\text { otillräckliga 1---2---3---4--8---7 tillräckliga }
$$

4. De hjälpmedel vi hade för att få översikt över beredskap för trafikolyckor var:

$$
\text { otillräckliga 1---2---3---4---6---7 tillräckliga }
$$

5. För att ge översikt över beredskap var kartan:

$$
\text { otydlig 1---2---3---4--- } 6 \text {---7 tydlig }
$$

6. För att ge översikt över beredskap var resursvyn:

$$
\text { otydlig 1---2---3--45---6---7 tydlig }
$$

7. Min egen koll på exakt varför beredskapen var bra / dålig / otillräcklig var:

$$
\text { otillfredsställande 1---2---3---4-8--6--7 tillfredställande }
$$

8. Vår egen prestation i stort var:

$$
\text { otillfredsställande 1---2---3---4-8-6---7 tillfredställande }
$$

9. Jag anser att de händelser som inträffade var upplagda på ett sätt som var:

$$
\text { orealistiskt 1---2---3---4--- }
$$

10. Jag anser att övningen i stort var:

$$
\text { orealistisk 1---2---3---4--- } 6 \text {---7 realistisk }
$$

